\title{
Parsimonious shooting heuristic for trajectory design of connected automated traffic part I: Theoretical analysis with generalized time geography
}

\author{
Fang Zhou ${ }^{\mathrm{a}}$, Xiaopeng $\mathrm{Li}^{\mathrm{b}}$, Jiaqi Ma ${ }^{\mathrm{a}}$ \\ a. Transportation Solutions and Technology Applications Division, \\ Leidos, Inc., Reston, VA 20190, USA \\ b.Department of Civil and Environmental Engineering, \\ University of South Florida, FL 33620, USA
}

\begin{abstract}
This paper studies a problem of designing trajectories of a platoon of vehicles on a highway segment with advanced connected and automated vehicle technologies. This problem is very complex because each vehicle trajectory is essentially an infinite-dimensional object and neighboring trajectories have complex interactions (e.g., car-following behavior). A parsimonious shooting heuristic algorithm is proposed to construct vehicle trajectories on an signalized highway segment that comply with the boundary condition for vehicle arrivals, vehicle mechanical limits, traffic lights and vehicle following safety. This algorithm breaks each vehicle trajectory into a few sections that each is analytically solvable. This decomposes the originally hard trajectory design problem to a simple constructive heuristic. Then we slightly adapt this shooting heuristic algorithm to efficiently solve a leading vehicle problem on an uninterrupted freeway. To study theoretical properties of the proposed algorithms, the time geography theory is generalized by considering finite accelerations. With this generalized theory, it is found that under mild conditions, these algorithms can always obtain a feasible solution to the original complex trajectory design problem. Further, we discover that the shooting heuristic solution is a generalization of the solution to the classic kinematic wave theory by incorporating finite accelerations. We identify the theoretical bounds to the difference between the shooting heuristic solution and the kinematic wave solution. Numerical experiments are conducted to verify the theoretical results and to draw additional managerial insights into the potential of trajectory design in improving traffic performance. In summary, this paper provides a methodological and theoretical foundation for advanced traffic control by optimizing the trajectories of connected and automated vehicles. Building upon this foundation, an optimization framework will be presented in a following paper as Part II of this study.
\end{abstract}

\section{Introduction}

\subsection{Background}

As illustrated by the trajectories in the time-space diagram in Figure 1(a), traffic on a signalized arterial is usually forced to decelerate and accelerate abruptly as a result of alternating green and red lights. When traffic density is relatively high, stop-and-go traffic patterns will be formed and propagated backwards along shock waves. Similar stop-and-go traffic also occurs frequently on freeways even without explicit signal interruptions. Such stop-and-go traffic imposes a number of adverse impacts to highway performance. Obviously, vehicles engaged in abrupt stop-and-go movements are exposed to a high crash risk (Hoffmann

\footnotetext{
${ }^{*}$ Corresponding author. Tel: 813-974-0778, E-mail: xiaopengli@usf.edu.
} 


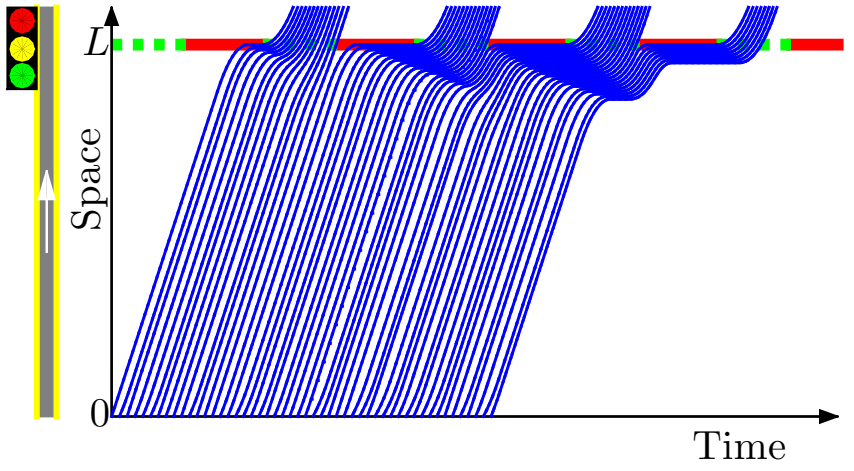

(a)

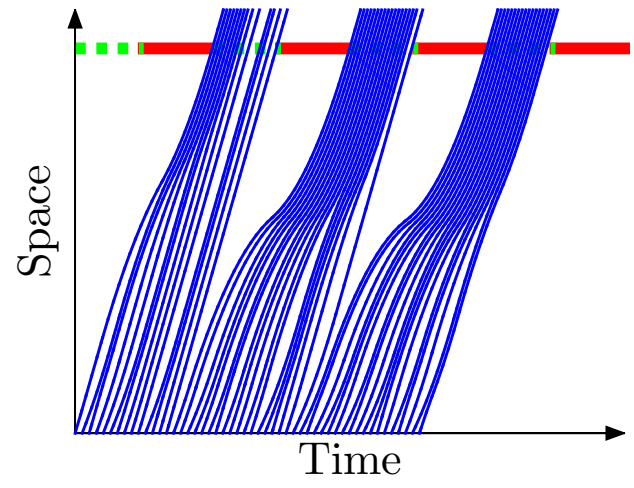

(b)

Figure 1: Vehicle trajectories along a highway segment upstream of an signalized intersection: (a) benchmark manual vehicle trajectories; (b) smoothed automated vehicle trajectories.

and Mortimer, 1994), not to mention extra discomfort to drivers (Beard and Griffin, 2013). Also, frequent decelerations and accelerations cause excessive fuel consumption and emissions ( $\mathrm{Li}$ et al., 2014), which pose a severe threat to the urban environment. Further, when vehicles slow down or stop, the corresponding traffic throughput decreases and the highway capacity drops (Cassidy and Bertini, 1999), which can cause excessive travel delay.

Although stop-and-go traffic has been extensively studied in the context of freeway traffic with either theoretical models (e.g., Herman et al. (1958); Bando et al. (1995); Li and Ouyang (2011)) or empirical observations (e.g., Kuhne (1987); Kerner and Rehborn (1996); Mauch and Cassidy (2002); Ahn (2005); Li and Ouyang (2011); Laval (2011a)), few studies had investigated how to smooth traffic and alleviate corresponding adverse consequences on signalized highways until the advent of vehicle-based communication (e.g., connected vehicles or CV) and control (e.g., automated vehicles or AV) technologies. CV basically enables real-time information sharing and communications among individual vehicles and infrastructure control units $^{1}$. AV aims to replace a human driver with a robot that constantly receives environmental information via various sensor technologies (as compared to human eyes and ears), and consequently determines vehicle control decisions (e.g., acceleration and braking) with proper computer algorithms (as compared to human brains) and vehicle control mechanics (as compared to human limbs) ${ }^{2}$. The combination of these two technologies, referred as connected and automated vehicles (CAV), enables disaggregated control (or coordination) of individual vehicles with real-time vehicle-to-vehicle and vehicle-to-infrastructure communications. Before these technologies, highway vehicle dynamics was determined by microscopic human driving behavior. However, there was not even a universally accepted formulation of human driving behavior (Treiber et al., 2010) due to the unpredictable nature of humans (Kerner and Rehborn, 1996) and limited empirical data to comprehensively describe such behavior (Daganzo et al., 1999). Therefore, it was very challenging, if not completely impossible, to perfectly smooth vehicle trajectories with traditional infrastructure-based controls (e.g., traffic signals) that are designed to accommodate human behavior. Whereas CAV enables the replacement (at least partially) of human drivers with programmable robots whose driving algorithms can be flexibly customized and accurately executed. This opens up opportunities to control individual vehicle trajectories in coordination with aggregate infrastructure-based controls such that both individual drivers' experience and overall traffic performance can be optimized. These opportunities inspired several pioneering studies to explore how to utilize CAVs to improve mobility and safety at intersections (Dresner and Stone, 2008; Lee and Park, 2012a) and reduce environmental impacts along highway segments (Ahn et al., 2013; Yang and Jin, 2014). However, these limited studies mostly focus on controlling one or very few vehicles at

\footnotetext{
${ }^{1}$ http://www.its.dot.gov/connected_vehicle/connected_vehicle.htm.

${ }^{2}$ http://en.wikipedia.org/wiki/Autonomous_car
} 
a particular highway facility (e.g., either an intersection or a segment) to achieve a certain specific objective (e.g., stability, safety or fuel consumption) rather than smoothing a stream of vehicles to improve the overall traffic performance. Most of the developed control algorithms require sophisticated numerical computations and their real-time applications might be hindered by excessive computational complexities.

This study aims to propose a new CAV-based traffic control framework that controls detailed trajectory shapes of a stream of vehicles on a stretch of highway combining a one-lane segment and a signalized intersection. As illustrated in Figure 1(b), the very basic idea of this study is smoothing vehicle trajectories and clustering them to platoons that can just properly occupy the green light windows and pass the intersection at a high speed. Note that a higher passing speed indicates a smaller car-following time headway or a larger throughput, and thus we see that the CAVs in Figure 1(b) not only have much smoother trajectories but also spend much less travel times compared with the benchmark manual vehicles in Figure 1(a). Further, smoother trajectories imply safer traffic, less fuel consumption and emissions, and better driver experiences. While the research idea is intuitive, the technical development is quite sophisticated, because this study needs to manipulate continuous trajectories that not only individually have infinite control points but also have complex interactions between one another due to the shared right-of-way. In order to overcome these modeling challenges, we first partition each trajectory into a few parabolic sections that each is analytically solvable. This essentially reduces an infinite-dimensional trajectory into a few set of parabolic function parameters. Further, we only use four acceleration and deceleration variables that are nonetheless able to control the overall smoothness of the whole stream of vehicle trajectories while assuring exceptional parsimony and simplicity of the proposed algorithm. With these treatments, we propose an efficient shooting heuristic algorithm that can generate a stream of smooth and properly platooned trajectories that can pass the intersection efficiently and safely yielding minimum environmental impacts. Also, note that some vehicle trajectory design problems on a freeway (e.g., CAV based speed harmonization and merging operations) can be adapted to the investigated problem as long as proper vehicle exit times and locations can be identified (e.g., by predicting shock waves and merging points). Since freeway traffic can be somehow viewed as arterial traffic with an infinite green phase, we investigate a lead vehicle problem for possible freeway adaptations of the proposed algorithms. After generalizing the concept of time geography (Miller, 2005) by allowing finite acceleration and deceleration, we discover a number of elegant properties of these algorithms in the feasibility to the original trajectory design problem and the connection with classic traffic flow models.

We want to note that this Part I paper only focuses on parsimonious algorithms for constructing a stream of feasible trajectories and their theoretical properties. These algorithms will serve as a building block for the comprehensive optimization framework presented in the Part II paper (Ma et al., 2015) that determines the best trajectories under several traffic performance measures, including travel time, fuel consumption, emissions and safety surrogate measures. While this paper also qualitatively discusses optimality issues with visual patterns in trajectory plots, we leave the detailed computational issues and the overall optimization framework to the Part II paper.

\section{$1.2 \quad$ Literature Review}

Freeway traffic smoothing has drawn numerous attentions from both academia and industry in the past several decades. Numerous studies have been conducted in attempts to characterize stop-and-go traffic on freeway (Herman et al., 1958; Chandler et al., 1958; Kuhne, 1987; Bando et al., 1995; Kerner and Rehborn, 1997; Bando et al., 1998; Kerner, 1998; Mauch and Cassidy, 2002; Ahn, 2005; Li and Ouyang, 2011; Laval, 2011a) Herman et al. (1958); Bando et al. (1995); Li and Ouyang (2011). However, probably due to the lack of high resolution trajectory data (Daganzo et al., 1999), no consensus has been formed on fundamental mechanisms of stop-and-go traffic formation and propagation, particularly at the microscopic level (Treiber et al., 2010). To harmonize freeway traffic speed, scholars and practitioners have proposed and tested a number of infrastructure-based control methods mostly targeting at aggregate traffic (rather than individual vehicles), including variable speed limits (Lu and Shladover, 2014), ramp metering(Hegyi et al., 2005), and merging traffic control (Spiliopoulou et al., 2009). While theoretical results show that these speed harmonization methods can drastically improve traffic performance in all major performance measures, e.g., safety, mobility and environmental impacts (Islam et al., 2013; Yang and Jin, 2014), field studies show the 
performances of these methods exhibit quite some discrepancies (Bham et al., 2010). Probably due to limited understanding of the microscopic behavior of highway traffic, these field practices of speed harmonization are mostly based on empirical experiences and trial-and-error approaches without taking full advantage of theoretical models. Also, drivers may not fully comply with the speed harmonization control and their individual responses may be highly stochastic, which further compromises the actual performance of these control strategies. Therefore, these aggregated infrastructure-based traffic smoothing measures may not perform as ideally as theoretical model predictions.

With the advancement of vehicle-based communication (i.e., CV) and control (i.e., AV) technologies, researchers started exploring ways of freeway traffic smoothing by controlling individual vehicles. Schwarzkopf and Leipnik (1977) analytically solved the optimal trajectory of a single vehicle on certain grade profiles with simple assumptions of vehicle characteristics based on Pontryagin's minimum principle. Hooker (1988) instead proposed a simulation approach that capture more realistic vehicle characteristics. Van Arem et al. (2006) finds that traffic flow stability and efficiency at a merge point can be improved by cooperative adapted cruise control (a longitudinal control strategy of CAV) that smooths car-following movements. Liu et al. (2012) solved an optimal trajectory problem for one single vehicle and used this trajectory as a template to control multiple vehicles with variable speed limits. Ahn et al. (2013) proposed a rolling-horizon individual CAV control strategy that minimizes fuel consumption and emission considering roadway geometries (e.g., grades). Yang and Jin (2014) proposed a vehicle speed control strategy to mitigate traffic oscillation and reduce vehicle fuel consumption and emission based on connected vehicle technologies. They found with only a 5 percent compliance rate, this control strategy can reduce traffic fuel consumption by up to 15 percent. Wang et al. (2014a; 2014b) proposed optimal control models based on dynamic programming and Pontryagin's minimum principle that determine accelerations of a platoon of AVs or CAVs to minimize a variety of objective cost functions. Li et al. (2014) revised a classic manual car-following model into one for $\mathrm{CAV}$ following by incorporating CAV features such as faster responding time and shared information. They found the CAV following rules can significantly reduce magnitudes of traffic oscillation, emissions and travel time. Tang et al. (2014a; 2014b) showed similar smoothing effects of CAV on car-following models. Despite relatively homogenous settings and complex algorithms, these adventurous developments have demonstrated a great potential of these advanced technologies in improving freeway mobility, safety and environment.

Despite these fruitful developments on the freeway side, traffic smoothing on interrupted highways (i.e., with at-grade intersections) is a relative recent concept. This concept is probably motivated by recent CAV technologies that allow vehicles' paths to be coordinated with signal controls. The existing traffic smoothing studies for interrupted highways can be in general categorized into two types. The first type assumes that CAVs can communicate with each other to pass an intersection in a self-organized manner (e.g., like a school of fish) even without conventional traffic signals. For example, Dresner and Stone (2008) proposed a heuristic control algorithm that process vehicles as a queuing system. While this development probably performs excellently when traffic is light or moderate, its performance under dense traffic is yet to be investigated. Further, Lee and Park (2012b) proposed a nonlinear optimization model to test the limits of a non-stop intersection control scheme. They showed that ideally, the optimal non-stop intersection control can significantly outperform classic signalized control in both mobility and environmental impacts at different congestion levels. Zohdy and Rakha (2014) integrated an embedded car-following rule and an intersection communication protocol into an nonlinear optimization model that manages a non-stop intersection. This model considers different weather conditions, heterogeneous vehicle characteristics and varying market penetrations. Overall, these developments on non-stop unsignalized control usually only focus on the operations of vehicles in the vicinity of an intersection and require complicated control algorithms and simulation. Implementing this complex mathematical programming model in real-time applications might require further investigations.

The second type of studies for interrupted traffic smoothing considers how to design vehicles trajectories in compliance with existing traffic signal controls at intersections. The basic idea is that a vehicle shall slow down when approaching a red light so that this vehicle might be able to pass the next green light following a relatively smooth trajectory without an abrupt stop. Trayford et al. (1984a; 1984b) tested using speed advice to vehicles approaching an intersection to reduce fuel consumption with computer simulation. 
Later studies extended the speed advice approach to car-following dynamics (Sanchez et al., 2006), in-vehicle traffic light assistance (Iglesias et al., 2008; Wu et al., 2010) multi-intersection corridors (Mandava et al., 2009; Guan and Frey, 2013; De Nunzio et al., 2013), scaled-up simulation (Tielert et al., 2010), and electric vehicles (Wu et al., 2015). These approaches mainly focused on the bulky part of a vehicle's trajectory with constant cruise speeds without much tuning its microscopic acceleration. However, acceleration details significantly affect a vehicle's fuel consumption and emissions (Rakha and Kamalanathsharma, 2011). To address this issue, Kamalanathsharma et al. (2013) proposes an optimization model that considers a more realistic yet more sophisticated fuel-consumption objective function in smoothing a single vehicle trajectory at a signalized intersection. As illustrated in Figure 1(b), the very basic idea of this study is smoothing vehicle trajectories and clustering them to platoons that can just properly occupy the green light windows and pass the intersection at a high speed. Note that a higher passing speed indicates a larger intersection capacity, and thus we see that the CAVs in Figure 1(b) not only have much smoother trajectories but also spend much less travel times compared with the benchmark manual vehicles in Figure 1(a). Further, smoother trajectories imply safer traffic, less fuel consumption and emissions, and better driver experiences. While the research idea is intuitive, the technical development is quite sophisticated, because this study needs to manipulate continuous trajectories that not only individually have infinite control points but also have complex interactions between one another due to the shared right-of-way. In order to overcome these modeling challenges, we first partition each trajectory into a few parabolic sections that each is analytically solvable. This essentially reduces an infinite-dimensional trajectory into a few set of parabolic function parameters. Further, we only use four acceleration and deceleration variables that are nonetheless able to control the overall smoothness of the whole stream of vehicle trajectories while assuring exceptional parsimony and simplicity of the proposed algorithm. With these treatments, we propose an efficient shooting heuristic algorithm that can generate a stream of smooth and properly platooned trajectories that can pass the intersection efficiently and safely yielding minimum environmental impacts. Also, note that this algorithm can be easily adapted to freeway speed harmonization as well because the freeway trajectory design problem is essentially a special case of the investigated problem with an infinite green time. We investigate a lead vehicle problem to study this freeway adaption. After generalizing the concept of time geography (Miller, 2005) by allowing finite acceleration and deceleration, we discover a number of elegant properties of these algorithms in the feasibility to the original trajectory design problem and the connection with classic traffic flow models. section. While such a model captures the advantage from microscopically tuning vehicle acceleration, it requires a complicated numerical solution algorithm that consumes a large amount of computation resources even for a single trajectory.

In summary, there have been increasing interests in vehicle smoothing using advanced vehicle-based technologies in recent years. However, most relevant studies only focus on controlling one or a few individual vehicles. Most studies in the trajectory-based traffic flow control literature either ignore acceleration detail and allow speed jumps to assure the model computational tractability, or capture acceleration in highly sophisticated algorithms that are difficult to implement in real time. Further, few studies investigated theoretical properties of the proposed controls and their relationships with classic traffic flow theories. Without such theoretical insights, we would miss the great opportunity of transferring the vast elegant developments of existing manual traffic in the past few decades to future CAV traffic.

The proposed trajectory optimization framework aims to fill these research gaps. We investigate a general trajectory design problem that optimizes individual trajectories of a long stream of interactive CAVs on a signalized highway segment. Note that the investigated problem assumes that all vehicles are CAV and subjected to centralized control. Although this assumption may not be consistent with the near future scenario where both automated and manual vehicles co-exist in highway traffic and these automated vehicles may not be subject to centralized control, the results from this assumption still provide valuable insights into the best the CAV technologies can achieve and also set an ultimate direction for the improvement of the current traffic with CAVs. This problem is general such that the lead vehicle problem on a freeway can be represented as its special case (e.g., by setting the red light time to zero). This problem is a very challenging infinite-dimension nonlinear optimization problem, and thus it is very hard to solve its exact optimal solution. We instead propose a heuristic shooting algorithm to solve a near-optimum solution to 
this problem. This algorithm can be easily extended to the freeway lead vehicle problem. The proposed shooting heuristic is different from a traditional shooting method for solving a boundary value problem (Stoer and Bulirsch, 2013; Von Stryk and Bulirsch, 1992). The proposed âĂIJshootingâĂİ means trajectory segments are constructed by shooting from both ends of the roadway segment and get tangent with each other at certain points in between. While the traditional shooting solution method implies shooting from initial conditions/guesses for multiple attempts, hoping to approach a given end boundary condition. This method is sensitive to initial guesses and leads to instability at highly dynamical situations in real-time control. However, our SH algorithm always yields a unique solution for a given set of parameters and inputs and is insensitive to initial errors. While the proposed algorithms can flexibly control trajectory shapes by tuning acceleration across a broad range, it is extremely parsimonious: it compresses a trajectory into a very few number of analytical segments, and includes only a few acceleration levels as control variables. With such parsimony and simplicity, these algorithms are suitable for real-time applications and further adaptations. The simple structure of these algorithms also allows us to analyze its theoretical properties. By extending the traditional time-geography theory to a second-order version that considers finite acceleration, we are able to analytically investigate the feasibility of the proposed algorithms and the implication to the feasibility of the original problem. This novel extension also allows us to relate the trajectories solved by the lead vehicle problem algorithm to those generated by classic traffic flow models. This helps us reveal the fundamental commonalities between these two highway traffic management paradigms based on completely different technologies. Note that the investigated trajectory optimization problem is essentially a short-term trajectory planning problem rather than a real-time control problem. While the real-time control can take our problem results as the input of planned trajectories for controlled vehicles to follow, it is a separate problem from the trajectory planning and thus will not be investigated in this study.

This paper is organized as follows. Section 2 states the studied CAV trajectory optimization problem on a signalized highway segment and its variant for the lead vehicle problem on a freeway segment. Section 3 describes the proposed shooting heuristic algorithms for the original problem and its variant, respectively. Section 4 analyzes the theoretical properties of the proposed algorithms based on an extended time-geography theory. Section 5 demonstrates the proposed algorithms and their properties with a few illustrative examples. Section 6 concludes this paper and briefly discusses future research directions.

\section{Problem Statement}

\subsection{Primary Problem}

This section describes the primary problem of trajectory construction on a signalized one-lane highway segment, as illustrated in Figure 2. We assume that this system is controlled by a centralized road side computer with perfect information (e.g., all information can be passed among this computer, the signal and the vehicles in real time with proper relays of V2I and V2V units) and communication delays (e.g., on the order of 1 second) are negligible compared with the trajectory design time horizon (e.g. on the order of 100 seconds). The problem setting is described below.

Roadway Geometry: We consider a single-lane highway segment of length $L$. Location on this segment starts from 0 at the upstream and ends at $L$ at the downstream. We use location set $[0, L]$ to denote this highway segment. Traffic goes from location 0 to $L$ on this segment. A fixed-time traffic light is installed at location $L$. Without loss of generality, a signal cycle is only composed of an effective green phase and an effective red phase, while the yellow time is split into these two phases (Branston and van Zuylen, 1978). The effective green phase starts at time 0 with an duration of $G$, followed by an effective red phase of duration $R$, and this pattern continues all the way. This indicates the signal cycle time is always $C:=R+G$. We denote the set of green time intervals as $\mathcal{G}:=\left\{\left[g_{m}:=m C, r_{m}:=m C+G\right)\right\}_{m \in \mathbb{Z}^{+}}$ where $\mathbb{Z}^{+}$is the non-negative integer set. We define function

$$
G(t)=\min \left\{t^{\prime} \in \mathcal{G}, t^{\prime}>t\right\}, \forall t \in[-\infty, \infty],
$$

which identifies the next closest green time to $t$. Note that $G(t)=t$ if $t \in \mathcal{G}$ or $G(t)>t$ if $t \notin \mathcal{G}$. 


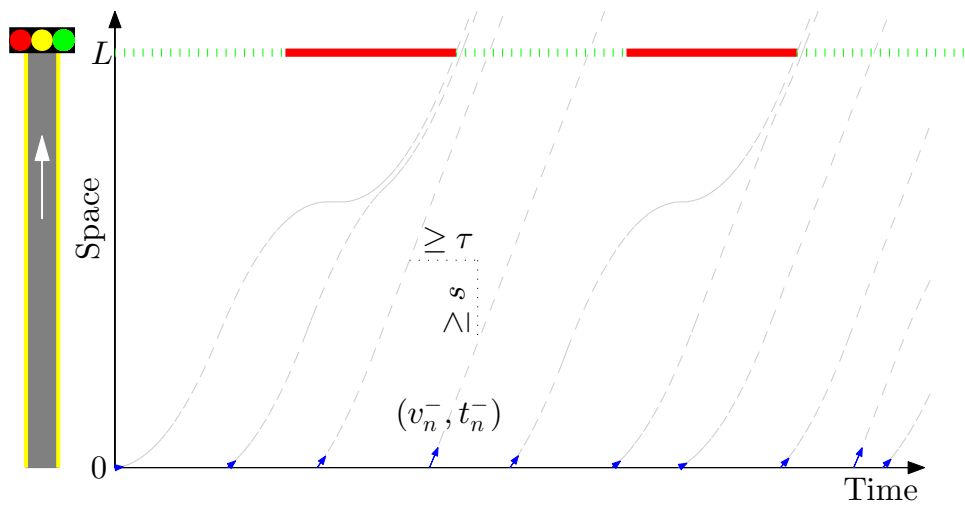

Figure 2: Illustration of the studied problem.

Vehicle Characteristics: We consider a stream of $N$ identical automated vehicles indexed as $n \in \mathcal{N}:=$ $\{1,2, \cdots, N\}$. Each vehicle's acceleration at any time is no less than deceleration limit $\underline{a}<0$ and no greater than acceleration limit $\bar{a}>0$. The speed limit on this segment is $\bar{v}$, and we don't allow a vehicle to back up, thus a vehicle's speed range is $[0, \bar{v}]$.

We want to design a set of trajectories for these vehicles to follow on this highway segment. A trajectory is formally defined below.

Definition 1. A trajectory is defined as a second-order semi-differentiable function $p(t), \forall t \in(-\infty, \infty)$ such that its first order differential (or velocity) $\dot{p}(t)$ is absolutely continuous and its second-order right-differential $\ddot{p}(t)$ (or acceleration) is Riemann integrable over any $t \in(-\infty, \infty)$. We denote the set of all trajectories by $\overline{\mathcal{T}}$. We call the subsection of $p$ between times $t^{-}$and $t^{+}\left(-\infty \leq t^{-}<t^{+} \leq \infty\right)$ a trajectory section, denoted by $p\left(t^{-}: t^{+}\right)$.

We let function $p_{n}$ denote the trajectory of vehicle $n, \forall n \in \mathcal{N}$. At any time $t, p_{n}(t)$ essentially denotes the location of vehicle $n$ 's front bumper. Collectively we denote all vehicle trajectories by trajectory vector $\mathbf{p}:=\left[p_{n}(t)\right]_{n \in \mathcal{N}}$. The trajectories in vector $\mathbf{p}$ shall satisfy the following constraints.

Kinematic Constraints: Trajectory $p$ is kinetically feasible if $\dot{p}(t) \in[0, \bar{v}]$ and $\ddot{p}(t) \in[\underline{a}, \bar{a}], \forall t \in(-\infty, \infty)^{3}$. We denote the set of all kinetically feasible trajectories as

$$
\mathcal{T}:=\{p \in \overline{\mathcal{T}} \mid 0 \leq \dot{p}(t) \leq \bar{v}, \underline{a} \leq \ddot{p}(t) \leq \bar{a}, \forall t \in(-\infty, \infty)\} .
$$

Entry Boundary Condition: Let $t_{n}^{-}$and $v_{n}^{-}$denote the time and the speed when vehicle $n$ arrives at the entry of this segment (or location 0 ), $\forall n \in \mathcal{N}$. We require $t_{1}^{-}<t_{2}^{-}<\cdots<t_{N}^{-}$and separation between $t_{n-1}$ and $t_{n}$ is sufficient for the safety requirement, $\forall n \in \mathcal{N} \backslash\{1\}$. Define the subset of trajectories in $\mathcal{T}$ that are consistent with vehicle $n$ 's entry boundary condition as

$$
\mathcal{T}_{n}^{-}:=\left\{p \in \mathcal{T} \mid p\left(t_{n}^{-}\right)=0, \dot{p}\left(t_{n}^{-}\right)=v_{n}^{-}\right\}, \forall n \in \mathcal{N} .
$$

Exit Boundary Condition: Due to the traffic signal at location $L$, vehicles can only exit this segment during a green light. Denote the set of trajectories in $\mathcal{T}$ that pass location $L$ during a green signal phase by

$$
\mathcal{T}^{+}:=\left\{p \in \mathcal{T} \mid p^{-1}(L) \in \mathcal{G}\right\}, \forall n \in \mathcal{N},
$$

where the generalized inverse function is defined as $p^{-1}(l):=\inf \{t \mid p(t) \geq l\}, \forall l \in[0, \infty), p \in \mathcal{T}$. Note that function $p^{-1}(\cdot)$ shall satisfy the following properties:

\footnotetext{
${ }^{3}$ In reality, the acceleration limit of a vehicle should be a decreasing function of speed. We set it as constant $\bar{a}$ just for the simplicity of the algorithm construction. Note that $\bar{a}$ should be set no more than the acceleration limit at the maximum speed $\bar{v}$ to assure its feasibility across the full speed range $[0, \bar{v}]$.
} 
P1: Function $p^{-1}(l)$ is increasing with $l \in(-\infty, \infty)$;

P2: Due to speed limit $\bar{v}, p^{-1}(l+\delta) \geq p^{-1}(l)+\delta / \bar{v}, \forall l \in(-\infty, \infty), \delta \in[0, \infty)$.

Further, let $\mathcal{T}_{n}$ denote the set of trajectories that satisfy both vehicle $n$ 's entry and exit boundary conditions, i.e., $\mathcal{T}_{n}=\mathcal{T}_{n}^{-} \cap \mathcal{T}^{+}$.

Car-following Safety: We require that the separation between vehicle $n$ 's location at any time and its preceding vehicle $(n-1)$ 's location a time delay $\tau$ ago is no less than a jam spacing $s, \forall n \in \mathcal{N} \backslash\{1\}$. Time $\tau$ is reserved to accommodate the responding delay between two consecutive vehicles; i.e., in case the preceding vehicle makes an emergency stop, it would take at maximum time $\tau$ for the following vehicle to properly respond. Spacing $s$ is composed of the vehicle length and a safety buffer that absorbs control errors. For a genetic trajectory $p \in \mathcal{T}$, a safely following trajectory of $p$ is a trajectory $p^{\prime} \in \mathcal{T}$ such that $p^{\prime}(t-\tau)-p(t) \geq s, \forall t \in(-\infty, \infty)$. We denote the set of all safely following trajectories of $p$ as $\mathcal{F}(p)$, i.e.,

$$
\mathcal{F}(p) \quad:=\quad\left\{p^{\prime} \mid p^{\prime}(t-\tau)-p(t) \geq s, \forall t \in(-\infty, \infty)\right\}, \forall p \quad \in \quad \quad \mathcal{T} .
$$

With this, we define $\mathcal{T}_{n}\left(p_{n-1}\right):=\mathcal{F}\left(p_{n-1}\right) \cap \mathcal{T}_{n}$ that denotes the set of feasible trajectories for vehicle $n$ that are safely following given vehicle $(n-1)$ 's trajectory $p_{n-1}$. We apply traffic safety constraint (4) because it is consistent with classic-crash free traffic flow models such as the kinematic wave model and Newell's simplified car-following model (see Section 4.4) while ensuring car-following safety in a physically sound way.

In summary, a feasible lead vehicle's trajectory $p_{1}$ has to fall in $\mathcal{T}_{1}$, and any feasible following vehicle trajectory $p_{n}$ has to belong to $\mathcal{T}_{n}\left(p_{n-1}\right), \forall n \in \mathcal{N} \backslash\{1\}$. We say a trajectory vector $\mathbf{p}$ is feasible if it satisfies all above-defined constraints. Let $\mathcal{P}$ denote the set of all feasible trajectory vectors, i.e.,

$$
\mathcal{P}:=\left\{\mathbf{p}:=\left[p_{n}\right]_{n \in \mathcal{N}} \mid p_{1} \in \mathcal{T}_{1}, p_{n} \in \mathcal{T}_{n}\left(p_{n-1}\right), \forall n \in \mathcal{N} \backslash 1\right\} .
$$

The investigated primary problem (PP) is to find and analyze feasible solutions to $\mathcal{P}$. This paper mainly focuses on proposing a constructive heuristic algorithm that solves a feasible solution to $\mathcal{P}$ and analyzing theoretical properties of this heuristic algorithm. The following Part II paper will build upon this heuristic algorithm and solve a trajectory optimization problem in the following form. Basically, once a feasible trajectory vector $\mathbf{p} \in \mathcal{P}$ is given, the traffic performance (e.g., in terms of travel time, fuel consumption and safety risks) can be essentially quantified as a function of $\mathbf{p}$, i.e., $M(\mathbf{p})$. Then the complete trajectory optimization problem is to solve the best trajectory vector $\mathbf{p} \in \mathcal{P}$ that optimizes the traffic performance, i.e., $\min _{\mathbf{p} \in \mathcal{P}} M(\mathbf{p})$. Since the theoretical analyses of PP in this paper are essentially stand-alone results and the following optimization problem can be viewed a separate problem just with the proposed heuristic as a component, we leave the detailed systematic description of this optimization problem to the Part II paper (Ma et al., 2015).

Remark 1. Although a realistic vehicle trajectory only has a limited length, we set a trajectory's time horizon to $(-\infty, \infty)$ to make the mathematical presentation convenient without loss of generality. In our study, we are only interested in trajectory sections between locations 0 and $L$, i.e., $p_{n}\left(t_{n}^{-}, p_{n}^{-1}(L)\right)$. Therefore, we can just view $p_{n}\left(-\infty, t_{n}^{-}\right)$as the given trajectory history that leads to the entry boundary condition, and $p_{n}\left(p_{n}^{-1}(L), \infty\right)$ as some feasible yet trivial projection above location $L$ (e.g., accelerating to $\bar{v}$ with rate $\bar{a}$ and then cruising at $\bar{v}$ ). Further, with this extension, safety constraint (4) also ensures that vehicles did not collide before arriving location 0 and will not collide after exiting location $L$.

Remark 2. This study can be trivially extended to deterministic yet time-variant signal timing; i.e., the signal timing plan is pre-determined, yet different cycles could have different green and red durations, e.g., alternating like $G_{1}, R_{1}, G_{2}, R_{2}, \cdots$. In this case, define signal timing switch points $g_{m}=\sum_{i=1}^{m}\left(G_{i}+R_{i}\right)$, $r_{m}=\sum_{i=1}^{m}\left(G_{i}+R_{i}\right)+G_{i}, \forall m \in \mathbb{Z}^{+}$and $\mathcal{G}$ becomes $\left\{\left[g_{m}, r_{m}\right)\right\}_{\forall m=1,2}$ where $r_{0}:=0$, and all the following results shall remain valid. 


\subsection{Problem Variation: Lead-Vehicle Problem}

In the classic traffic flow theory, the lead-vehicle problem (LVP) is a well-known fundamental problem that predicts traffic flow dynamics on one-lane freeway given the lead vehicle's trajectory and the following vehicle's initial states (Daganzo, 2006). We notice that PP (5) can be easily adapted to LVP by relaxing exit boundary condition (3) yet fixing trajectory $p_{1}$. The LVP is officially formulated as follows. Given lead vehicle's trajectory $p_{1} \in \mathcal{T}$, the set of feasible trajectories for LVP is

$$
\mathcal{P}^{\mathrm{LVP}}\left(p_{1}\right):=\left\{\mathbf{p}:=\left[p_{n}\right]_{n \in \mathcal{N}} \mid p_{n} \in \mathcal{F}\left(p_{n-1}\right) \bigcap \mathcal{T}_{n}^{-}, \forall n \in \mathcal{N} \backslash 1\right\}
$$

The LVP investigated in this paper is finding and analyzing feasible solutions to $\mathcal{P}^{\mathrm{LVP}}$.

\section{Shooting Heuristic Algorithms}

This section proposes customized heuristic algorithms to solve feasible trajectory vectors to PP and LVP. Although a trajectory is defined over the entire time horizon $(-\infty, \infty)$, these algorithms only focus on the trajectory sections from the entry time $t_{n}^{-}$for each vehicle $n \in \mathcal{N}$, because the trajectory sections before $t_{n}^{-}$should be trivial given history and do not affect the algorithm results. Therefore, in the following presentation, we view a trajectory and the corresponding trajectory section over time $\left[t_{n}^{-}, \infty\right)$ the same.

\subsection{Shooting Heuristic for PP}

This section presents a shooting heuristic ( $\mathrm{SH}$ ) algorithm that is able to construct a smooth and feasible trajectory vector to solve $\mathcal{P}$ in PP efficiently. Traditional methods for trajectory optimization include analytical approaches that can only solve simple problems with special structures and numerical approaches that can accommodate more complex settings yet may demand enormous computational resources (Von Stryk and Bulirsch, 1992). Since a vehicle trajectory is essentially an infinite-dimensional object along which the state (e.g. location, speed, acceleration) at every point can be varied, it is challenging to even construct one single trajectory, particularly under nonlinear constraints. Note that our problem deals with a large number of trajectories for vehicles in a traffic stream that constantly interact with each other and are subject to complex nonlinear constraints (1)-(5). Therefore we deem that it is very complex and time-consuming to tackle this problem with a traditional approach. Therefore, we opt to devise a new approach that circumvents the need for formulating high-dimensional objects or complex system constraints. This leads to the development of a shooting heuristic ( $\mathrm{SH}$ ) algorithm that can efficiently construct a smooth feasible trajectory vector with only a few control parameters.

Figure 3 illustrates the components in the proposed $\mathrm{SH}$ algorithm. Basically, for each vehicle $n \in \mathcal{N}, \mathrm{SH}$ first constructs a trajectory, denoted by $p_{n}^{\mathrm{f}}$, with a forward shooting process that conforms with kinematic constraint (1), entry boundary constraint (2) and safety constraint (4) (if $n>1$ ). As illustrated in Figure $3(\mathrm{a})$, if trajectory $p_{n}^{\mathrm{f}}$ (dashed blue curve) turns out far enough from preceding trajectory $p_{n-1}$ (red solid curve) such that safety constraint (4) is even not activated (or if $n=1$ and $p_{n}^{\mathrm{f}}$ is already the lead trajectory), $p_{n}^{f}$ basically accelerates from its entry boundary condition $\left(t_{n}^{-}, v_{n}^{-}\right)$at location 0 with a forward acceleration rate of $\bar{a}^{\mathrm{f}} \in(0, \bar{a}]$ until reaching speed limit $\bar{v}$ and then cruises at constant speed $\bar{v}$. Otherwise, as illustrated in Figure 3(b), if trajectory $p_{n}^{\mathrm{f}}$ is blocked by $p_{n-1}$ due to safety constraint (4), we let $p_{n}^{\mathrm{f}}$ smoothly merge into a safety bound (the red dotted curve) translated from $p_{n-1}$ that just keeps spatial separation $s$ and temporal separation $\tau$ from $p_{n}^{\mathrm{f}}$. The transitional segment connecting $p_{n}^{\mathrm{f}}$ with the safety bound decelerates at a forward deceleration rate of $\underline{a}^{\mathrm{f}} \in[\underline{a}, 0)$. If trajectory $p_{n}^{\mathrm{f}}$ from the forward shooting process is found to violate exit boundary constraint (3) (or run into the red light), as illustrated in Figure 3(c), a backward shooting process is activated to revise $p_{n}^{\mathrm{f}}$ to comply with constraint (3). The backward shooting process first shifts the section of $p_{n}^{\mathrm{f}}$ above location $L$ rightwards to the start of the next green phase to be a backward shooting trajectory $p_{n}^{\mathrm{b}}$. Then $p_{n}^{\mathrm{b}}$ shoots backwards from this start point at a backward acceleration rate $\bar{a}^{\mathrm{b}} \in(0, \bar{a}]$ until getting 


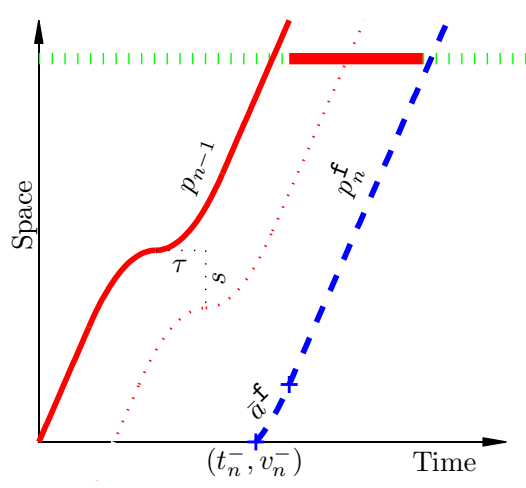

(a)

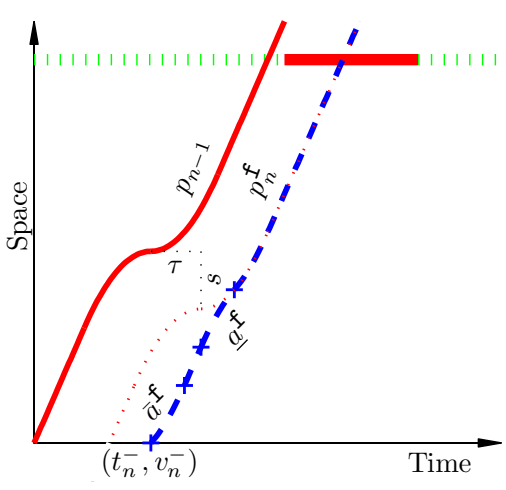

(b)

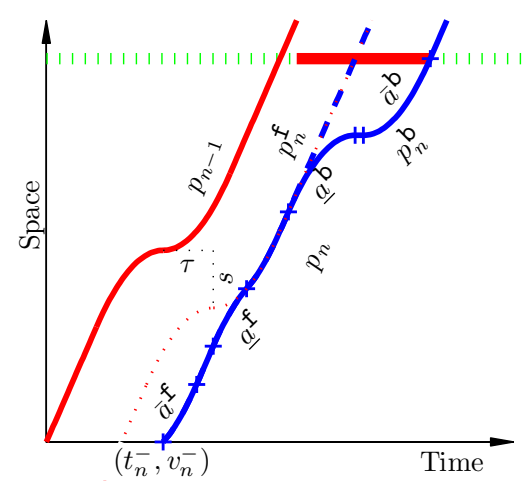

(c)

Figure 3: Components in the proposed shooting heuristic: (a) forward shooting process without activating safety constraint (4); (b) forward shooting process with activating safety constraint (4); and (c) backward shooting process. .

close enough to merge into $p_{n}^{\mathrm{f}}$, which may require $p_{n}^{\mathrm{b}}$ stops for some time if the separation between the backward shooting start point and $p_{n}^{\mathrm{f}}$ is long relative to acceleration rate $\bar{a}^{\mathrm{b}}$. Then, $p_{n}^{\mathrm{b}}$ shoots backwards a merging segment at a backward deceleration rate $\underline{a}^{\mathrm{b}} \in[\underline{a}, 0)$ until getting tangent to $p_{n}^{\mathrm{f}}$. Finally, merging $p_{n}^{\mathrm{f}}$ with $p_{n}^{\mathrm{b}}$ yields a feasible trajectory $p_{n}$ for vehicle $n$. Such forward and backward shooting processes are executed from vehicle 1 through vehicle $N$ consecutively, and then SH concludes with a feasible trajectory vector $\mathbf{p}=\left[p_{n}\right]_{n \in \mathcal{N}} \in \mathcal{P}$. Note that $\mathrm{SH}$ uses only four control variables $\left\{\bar{a}^{\mathrm{f}}, \underline{a}^{\mathrm{f}}, \bar{a}^{\mathrm{b}}, \underline{a}^{\mathrm{b}}\right\}$ that are yet able to control the overall smoothness of all trajectories (so as to achieve certain desired objectives). Further, the constructed trajectories are composed of only a few quadratic (or linear) segments that are all analytically solvable. Therefore, the proposed SH algorithm is parsimonious and simple to implement.

To formally state SH, we first define the following terminologies in Definitions 2-4 with respect to a single trajectory, as illustrated in Figure 4(a).

Definition 2. We define a state point by a three-element tuple $\left(l, v, t^{\prime}\right)$, which represents that at time $t^{\prime}$, the vehicle is at location $l$ and operates at speed $v$. A feasible state point $\left(l, v, t^{\prime}\right)$ should satisfy $v \in[0, \bar{v}]$.

Definition 3. We use a four-element tuple $\left(l, v, a, t^{\prime}\right)$ to denote the quadratic function that passes location $l$ at time $t^{\prime}$ with velocity $v$ and acceleration $a$; i.e., $0.5 a\left(t-t^{\prime}\right)^{2}+v\left(t-t^{\prime}\right)+l$ with respect to time variable $t \in(-\infty, \infty)$. Note that this quadratic function definition also includes a linear function (i.e., $a=0)$. For simplicity, we can use a boldface letter to denote a quadratic function, e.g., $\mathbf{f}:=\left(l, v, a, t^{\prime}\right)$ and $\mathbf{f}(t):=$ $0.5 a\left(t-t^{\prime}\right)^{2}+v\left(t-t^{\prime}\right)+l$.

Definition 4. We use a five-element tuple $\mathbf{s}:=\left(l, v, a, t^{\prime}, t^{\prime \prime}\right)$ to denote a segment of quadratic function $\mathbf{f}=\left(l, v, a, t^{\prime}\right)$ between time $\min \left\{t^{\prime}, t^{\prime \prime}\right\}$ and $\max \left\{t^{\prime}, t^{\prime \prime}\right\}$. We call this tuple a quadratic segment. Define $\mathbf{s}(t):=\mathbf{f}(t), \forall t \in\left[\min \left\{t^{\prime}, t^{\prime \prime}\right\}, \max \left\{t^{\prime}, t^{\prime \prime}\right\}\right]$. If the speed and the acceleration on every point along this segment satisfy constraint (1), we call it a feasible quadratic segment.

Remark 3. In Definitions 2-4, if one or more elements in a tuple are unknown or variable, we use "." to hold their places (e.g., $\left.\left(l, \cdot, t^{\prime}\right),\left(l, v, \cdot, t^{\prime}, \cdot\right)\right)$.

Definition 5. For a trajectory $p \in \overline{\mathcal{T}}$ composed by a vector of consecutive quadratic segments $\mathbf{s}_{k}:=$ $\left[\left(l_{k}, v_{k}, a_{k}, t_{k}, t_{k+1}\right)\right]_{k=1,2, \cdots, \bar{k}}$ with $-\infty=t_{1}<t_{2}<\cdots<t_{\bar{k}+1}=\infty$, we denote $p=\left[\mathbf{s}_{k}\right]_{k=1,2, \cdots, \bar{k}}$. Without loss of generality, we assume that any trajectory in this study can be decomposed into a vector of quadratic segments. 


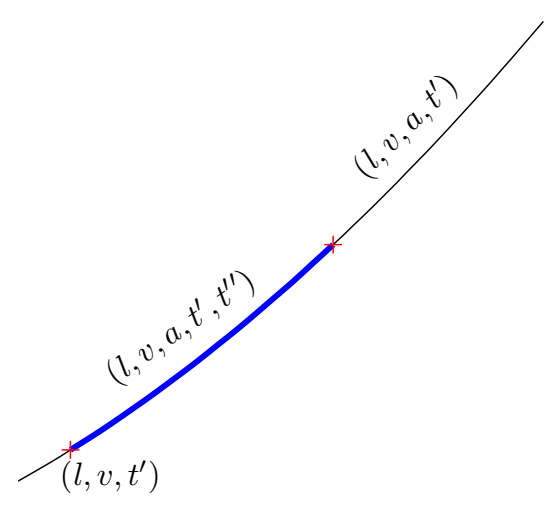

(a)

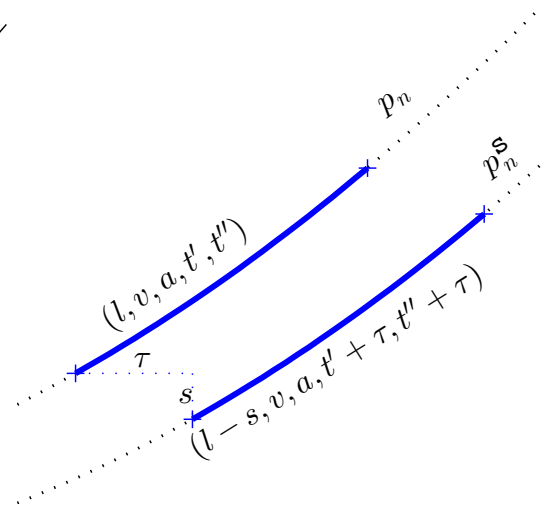

(b)

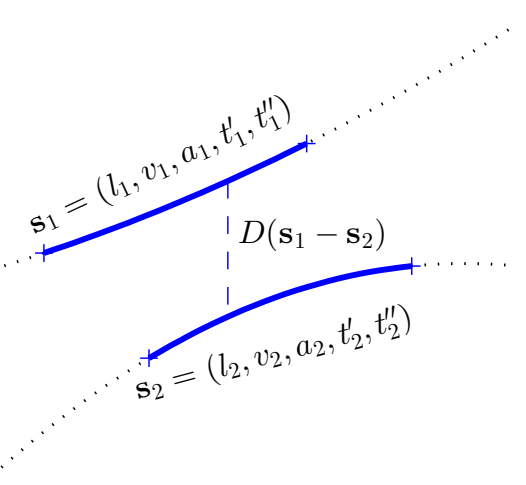

(c)

Figure 4: Illustrations of definitions ( $x$-axis for time elapsing rightwards and $y$-axis for location increasing upwards): (a) state point, quadratic function and segment; (b) shadow trajectory and shadow segment; and (c) segment distance. .

During the forward shooting process, we need to check safety constraints (4) at every move for any vehicle $n \geq 2$. As illustrated in Figure 4(b), we basically create a shadow (or safety bound) of the preceding trajectory $p_{n-1}$ by shifting $p_{n-1}$ downwards by $s$ and rightwards by $\tau$. Then safety constraint (4) is simply equivalent to that $p_{n}$ does not exceed this shadow trajectory at any time. The following definition specifies the shadow trajectory and its elemental segments.

Definition 6. For a trajectory $p_{n}(t), \forall t \in(-\infty, \infty)$, we define its shadow trajectory $p_{n}^{\mathrm{S}}$ as $p_{n}^{\mathrm{S}}(t):=p_{n}(t-\tau)-$ $s, \forall t \in(-\infty, \infty)$. It is obvious that $t^{-}\left(p_{n}^{\mathrm{S}}\right)=t_{n}^{-}+\tau$ and $t^{+}\left(p_{n}^{\mathrm{S}}\right)=\infty$. Note that if the following trajectory $p_{n+1}(t)$ initiated at time $t_{n+1}^{-}$satisfies $p_{n+1}(t) \leq p_{n}^{\mathrm{S}}, \forall t \in(-\infty, \infty)$, then $p_{n}$ and $p_{n+1}$ satisfies safety constraint (4). Further, a shadow segment of $\mathbf{s}:=\left(l, v, a, t^{\prime}, t^{\prime \prime}\right)$ is simply $\mathbf{s}^{\mathrm{S}}:=\left(l-s, v, a, t^{\prime}+\tau, t^{\prime \prime}+\tau\right)$. We also generalize this definition to the $m^{\text {th }}$-order shadow trajectory of $p_{n}$ as $p_{n}^{\mathrm{S}^{m}}(t):=p_{n}(t-m \tau)-s m, \forall t \in(-\infty, \infty)$ and $m^{\text {th }}$-order shadow segment $\mathbf{s}^{\mathbf{S}^{m}}:=\left(l-m s, v, a, t^{\prime}+m \tau, t^{\prime \prime}+m \tau\right)$, which are essentially the results of repeating the shadow operation by $m$ times.

The following definitions specify an analytical function that checks the distance between two segments (e.g., the current segment to be constructed and a reference shadow segment in forward shooting), as illustrated in Figure 4(c).

Definition 7. Given two segments $\mathbf{s}_{1}:=\left(l_{1}, v_{1}, a_{1}, t_{1}^{\prime}, t_{1}^{\prime \prime}\right), \mathbf{s}_{2}:=\left(l_{2}, v_{2}, a_{2}, t_{2}^{\prime}, t_{2}^{\prime \prime}\right)$, define segment distance from $\mathbf{s}_{1}$ to $\mathbf{s}_{2}$ as

$$
D\left(\mathbf{s}_{1}-\mathbf{s}_{2}\right):= \begin{cases}\min _{t \in\left[t^{-}, t^{+}\right]} \mathbf{s}_{1}(t)-\mathbf{s}_{2}(t), & \text { if } t^{-} \leq t^{+} \\ \infty, & \text { otherwise }\end{cases}
$$

where $t^{-}:=\max \left\{\min \left\{t_{1}^{\prime}, t_{1}^{\prime \prime}\right\}, \min \left\{t_{2}^{\prime}, t_{2}^{\prime \prime}\right\}\right\}$ and $t^{+}:=\min \left\{\max \left\{t_{1}^{\prime}, t_{1}^{\prime \prime}\right\}, \max \left\{t_{2}^{\prime}, t_{2}^{\prime \prime}\right\}\right\}$. If $t^{-} \leq t^{+}, D\left(\mathbf{s}_{1}-\mathbf{s}_{2}\right)$ can be solved analytically as

$$
D\left(\mathbf{s}_{1}-\mathbf{s}_{2}\right):= \begin{cases}\mathbf{s}_{1}\left(t^{*}\left(\mathbf{s}_{1}-\mathbf{s}_{2}\right)\right)-\mathbf{s}_{2}\left(t^{*}\left(\mathbf{s}_{1}-\mathbf{s}_{2}\right)\right), \text { if } a_{1}-a_{2}>0 \text { and } t^{*}\left(\mathbf{s}_{1}-\mathbf{s}_{2}\right) \in\left(t^{-}, t^{+}\right) \\ \min \left\{\mathbf{s}_{1}\left(t^{-}\right)-\mathbf{s}_{2}\left(t^{-}\right), \mathbf{s}_{1}\left(t^{+}\right)-\mathbf{s}_{2}\left(t^{+}\right)\right\} & \text {otherwise. }\end{cases}
$$

where

$$
t^{*}\left(\mathbf{s}_{1}-\mathbf{s}_{2}\right):=\frac{v_{1}-a_{1} t_{1}^{\prime}-v_{2}+a_{2} t_{2}^{\prime}}{a_{2}-a_{1}} .
$$


We also extend the distance definition to trajectory sections and trajectories below. Given two trajectory sections $p\left(t^{-}: t^{+}\right)$and $p^{\prime}\left(t^{\prime-}: t^{\prime+}\right)$, the distance from $p\left(t^{-}: t^{+}\right)$to $p^{\prime}\left(t^{\prime-}: t^{\prime+}\right)$ is defined as

$$
D\left(p\left(t^{-}: t^{+}\right)-p^{\prime}\left(t^{\prime-}: t^{\prime+}\right)\right):=\min _{t \in\left[\max \left(t^{-}, t^{\prime}\right), \min \left(t^{+}, t^{+}\right)\right]} p(t)-p^{\prime}(t)
$$

if $\max \left(t^{-}, t^{\prime-}\right) \leq \min \left(t^{+}, t^{\prime+}\right)$ or $D\left(p\left(t^{-}: t^{+}\right)-p^{\prime}\left(t^{\prime-}: t^{\prime+}\right)\right):=\infty$. Suppose these two sections can be partitioned into quadratic segments, i.e., $p\left(t^{-}: t^{+}\right)=\left[\mathbf{s}_{k}\right]_{k=1,2, \cdots, \bar{k}}$ and $p^{\prime}\left(t^{\prime-}: t^{\prime+}\right)=\left[\mathbf{s}_{k^{\prime}}^{\prime}\right]_{k^{\prime}=1,2, \cdots, \bar{k}^{\prime}}$, then

$$
D\left(p\left(t^{-}: t^{+}\right)-p^{\prime}\left(t^{\prime-}: t^{\prime+}\right)\right)=\min _{k=1, \cdots, \bar{k}, k^{\prime}=1, \cdots, \bar{k}^{\prime}} D\left(\mathbf{s}_{k}-\mathbf{s}_{k^{\prime}}^{\prime}\right) .
$$

Note that function $D(\cdot)$ has a transitive relationship; i.e., $D(A-B) \geq D_{A B}$ and $D(B-C) \geq D_{B C}$ indicates $D(A-C) \geq D_{A B}+D_{B C}$.

Definition 8. Next, we define an analytical operation that determines how we take a move in the forward shooting process, as illustrated in Figure 5(a). Given a quadratic segoverallment $\mathbf{s}^{\prime}:=\left(l^{\prime}, v^{\prime}, a^{\prime}, t^{\prime-}, t^{\prime+}\right)$ with $t^{\prime-}<t^{\prime+}$, a feasible state point $\left(l, v, t^{-}\right)$with $t^{-}<t^{\prime+}$, acceleration rate $a^{+} \geq 0$ and deceleration rate $a^{-}<0$ (and $a^{-} \leq a^{\prime}$ ), we want to construct a forward shooting segment $\mathbf{s}:=\left(l, v, a^{+}, t^{-}, t^{\mathrm{m}}\right.$ ) followed by a forward merging segment $\mathrm{s}^{\mathrm{m}}:=\left(l^{\mathrm{m}}, v^{\mathrm{m}}, a^{-}, t^{\mathrm{m}}, t^{+}\right)$where $v^{\mathrm{m}}:=v+a^{+}\left(t^{\mathrm{m}}-t^{-}\right), l^{\mathrm{m}}:=p+v\left(t^{\mathrm{m}}-t^{-}\right)+$ $0.5 a^{+}\left(t^{\mathrm{m}}-t^{-}\right)^{2}$ with $t^{+} \geq t^{\mathrm{m}} \geq t^{-}$in the following way. We basically select $t^{-}$and $t^{m}$ values to make $\mathbf{s}$ and $\mathbf{s}^{\mathrm{m}}$ satisfy the following conditions. First, we want to keep $\mathbf{s}^{\prime}$ above $\mathbf{s}$ and the segment extended from $\mathrm{s}^{\mathrm{m}}$ to time $\infty$, i.e.,

$$
D\left[\mathbf{s}^{\prime}-\mathbf{s}\right] \geq 0, \text { and } D\left[\mathbf{s}^{\prime}-\left(p^{\mathrm{m}}, v^{\mathrm{m}}, a^{-}, t^{\mathrm{m}}, \infty\right)\right] \geq 0 .
$$

Further, the exact values of $t^{\mathrm{m}}$ and $t^{+}$shall be determined in the following three cases: (I) if no $t^{\mathrm{m}} \in\left[t^{-}, \infty\right)$ can be found to satisfy constraint (8), this shooting operation is infeasible and return $t^{m}=t^{+}=-\infty$; (II) otherwise, we try to find $t^{+} \in\left[\max \left\{t^{\prime-}, t^{-}\right\}, t^{\prime+}\right]$ and $t^{\mathrm{m}} \in\left[t^{-}, t^{+}\right]$such that $\mathbf{s}^{\prime}$ and $\mathbf{s}^{\mathrm{m}}$ get tangent at time $t^{+}$; and (III) if this trial fails, set $t^{\mathrm{m}}=t^{+}=\infty$. Fortunately, since these segments are all simple quadratic segments, $t^{\mathrm{m}}$ and $t^{+}$can be solved analytically in the following forward shooting operation (FSO) algorithm, where the final solutions to $t^{\mathrm{m}}$ and $t^{+}$are denoted by $t^{\mathrm{mf}}\left(\mathbf{s}^{\prime},\left(l, v, t^{-}, a^{+}\right), a^{-}\right)$and $t^{+\mathrm{f}}\left(\mathbf{s}^{\prime},\left(l, v, t^{-}, a^{+}\right), a^{-}\right)$, respectively.

FSO-1: If $D\left(\mathbf{s}^{\prime}-\overline{\mathbf{s}}\right)<0$ where $\overline{\mathbf{s}}:=\left(l, v, a^{-}, t^{-}, \infty\right)$, there is no feasible solution, and we just set $t^{\mathrm{m}}=$ $t^{+}=-\infty$ (Case I). Go to Step FSO-3.

FSO-2: Shift the origin to time $t^{-}$and denote $\hat{t}^{\prime-}:=t^{\prime-}-t^{-}, \hat{t}^{\prime+}:=t^{\prime+}-t^{-}, \hat{t}^{\mathrm{m}}:=t^{\mathrm{m}}-t^{-}, \hat{t}^{+}:=$ $t^{+}-t^{-}$and $\hat{t}^{-}:=\max \left\{\hat{t}^{\prime-}, 0\right\}$. Then get a quadratic function $\mathbf{q}$ by subtracting $\left(l^{\mathrm{m}}, v^{\mathrm{m}}, a^{-}, t^{\mathrm{m}}\right)$ from $\left(l^{\prime}, v^{\prime}, a^{\prime}, t^{\prime-}\right)$, i.e., $\mathbf{q}:=\left(\hat{l}, \hat{v}, a^{\prime}-a^{-}, 0\right)$ where

$$
\hat{l}:=0.5 a^{\prime}\left(\hat{t}^{\prime-}\right)^{2}+0.5\left(a^{+}-a^{-}\right)\left(\hat{t}^{\mathrm{m}}\right)^{2}-v^{\prime} \hat{t}^{\prime-}+l^{\prime}-l,
$$

and

$$
\hat{v}=v^{\prime}-v-a^{\prime} \hat{t}^{\prime-}-\left(a^{+}-a^{-}\right) \hat{t}^{\mathrm{m}} .
$$

FSO-2-1: If $a^{\prime}=a^{-}$, test whether we can make $\mathbf{q}(t)=0, \forall t \in\left[\hat{t}^{-}, \infty\right)$, i.e., whether $\hat{l}=0$ with $\hat{t}^{\mathrm{m}}=$ $\left(v^{\prime}-v-a^{\prime} \hat{t}^{\prime}\right) /\left(a^{+}-a^{-}\right)$. If yes and $\hat{t}^{\mathrm{m}} \in\left[\hat{t}^{-}, \hat{t}^{\prime+}\right]$ (Case II), set $t^{\mathrm{m}}=t^{-}+\hat{t}^{\mathrm{m}}$ and $t^{+}=t^{\prime+}$. Otherwise (Case III), set $t^{+}=t^{\mathrm{m}}=\infty$. Go to Step FSO-3.

FSO-2-2: If $a^{\prime}>a^{-}$,then $\mathbf{q}$ is a convex quadratic function, and we need to solve $\alpha\left(\hat{t}^{\mathrm{m}}\right)^{2}+\beta \hat{t}^{\mathrm{m}}+\gamma=0$ where $\alpha:=\left(a^{+}-a^{-}\right)\left(a^{+}-a^{\prime}\right), \beta:=-2\left(a^{+}-a^{-}\right)\left(v^{\prime}-v-a^{\prime} \hat{t}^{\prime}\right)$ and $\gamma:=\left(v^{\prime}-v-a^{\prime} \hat{t}^{-}\right)^{2}-\left(a^{\prime}-\right.$ $\left.a^{-}\right)\left(a^{\prime}\left(\hat{t}^{\prime-}\right)^{2}-2 v^{\prime} \hat{t}^{\prime-}+2 l^{\prime}-2 l\right)$. In case of $\alpha=\beta=0$ (Case III), set $t^{+}=t^{\mathrm{m}}=\infty$, and go to 


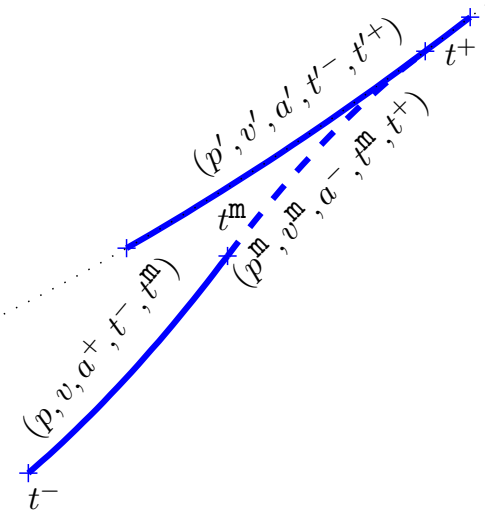

(a)

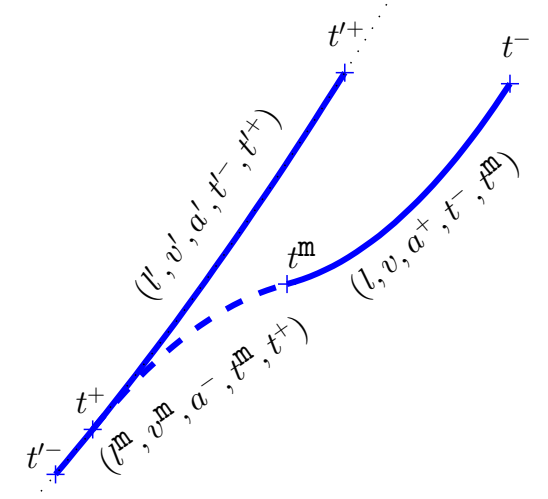

(b)

Figure 5: Illustrations of shooting operations: (a) a forward shooting operation, and (b) a backward shooting operation. .

Step FSO-3. Otherwise, we need to try candidate solutions to $\hat{t}^{\mathrm{m}}$ and $\hat{t}^{+}$, denoted by $\hat{t}^{\mathrm{mc}}$ and $\hat{t}^{+\mathrm{c}}$, respectively. In case of $\alpha=0$ but $\beta \neq 0$, solve $\hat{t}^{\mathrm{mc}}=-\gamma / \beta$ and

$$
\hat{t}^{+\mathrm{C}}=\frac{v^{\prime}-v-a^{\prime} \hat{t}^{\prime-}-\left(a^{+}-a^{-}\right) \hat{t}^{\mathrm{mc}}}{a^{-}-a^{\prime}} .
$$

Otherwise, we should have $\alpha \neq 0$, and then solve both candidate solutions

$$
\hat{t}^{\mathrm{mc}}=\frac{-\beta \pm \sqrt{\beta^{2}-4 \alpha \gamma}}{2 \alpha}
$$

and the corresponding $\hat{t}^{+\mathrm{C}}$ with equation(9). If the candidate solutions are not real numbers, then we just set $\hat{t}^{+\mathrm{c}}=\hat{t}^{\mathrm{mc}}=\infty$. Otherwise, try both sets of solutions and select the set satisfying $\hat{t}^{+\mathrm{c}} \geq \hat{t}^{\mathrm{mc}} \geq 0$. For either of these two cases, if $\hat{t}^{+\mathrm{c}} \in\left[\hat{t}^{-}, \hat{t}^{\prime+}\right]$ (Case II), we set $t^{\mathrm{m}}=t^{-}+\hat{t}^{\mathrm{mc}}$ and $\hat{t}^{+}=\hat{t}^{+\mathrm{c}}$. Otherwise, set $t^{+}=t^{\mathrm{m}}=\infty$ (Case III). Go to Step FSO-3.

FSO-3: Finally, we return $t^{\mathrm{mf}}\left(\mathbf{s}^{\prime},\left(l, v, t^{-}, a^{+}\right), a^{-}\right)=t^{\mathrm{m}}$ and $t^{+\mathrm{f}}\left(\mathbf{s}^{\prime},\left(l, v, t^{-}, a^{+}\right), a^{-}\right)=t^{+}$.

Definition 9. We extend one forward shooting operation to the following forward shooting process (FSP) that generates a whole trajectory. Given a shadow trajectory $p^{\mathrm{S}}:=\left[\mathbf{s}_{h}^{\mathrm{S}}:=\left(l_{h}^{\mathrm{S}}, v_{h}^{\mathrm{S}}, a_{h}^{\mathrm{S}}, t_{h}^{\mathrm{S}}, t_{h+1}^{\mathrm{S}}\right)\right]_{h=1, \cdots, \bar{h}}$. (with $t_{1}^{\mathrm{S}}=-\infty$ and $t_{\bar{h}+1}^{\mathrm{S}}=\infty$ ) and a feasible entry state point $\left(l, v, t^{-}\right)$, we want to construct a forward shooting trajectory $p^{\mathrm{f}}\left(\left(l, v, t^{-}\right), p^{\mathrm{S}}\right)$ that starts from $\left(l, v, t^{-}\right)$and maintains acceleration $\bar{a}^{\mathrm{f}}$ or speed $\bar{v}$ until being bounded by $p^{\mathrm{S}}$. We consider a template trajectory starting at $\left(l, v, t^{-}\right)$and composed by these two candidate segments $\mathbf{s}^{\mathrm{a}}:=\left(l, v, \bar{a}^{\mathrm{f}}, t^{-}, t^{\mathrm{a}}:=t^{-}+(\bar{v}-v) / \bar{a}^{\mathrm{f}}\right)$ (which accelerates from $v$ to $\bar{v}$ given $v<\bar{v}$ ) and $\mathbf{s}^{\infty}:=\left(l^{\mathrm{a}}:=l+0.5\left(\bar{v}^{2}-v^{2}\right) / \bar{a}^{\mathrm{f}}, \bar{v}, 0, t^{\mathrm{a}}, \infty\right)$ (which maintains maximum speed $\bar{v}$ all the way), i.e.,

$$
p^{\mathrm{t}}:= \begin{cases}{\left[\mathbf{s}^{\mathrm{a}}, \mathbf{s}^{\infty}\right],} & \text { if } v<\bar{v} \\ {\left[\mathbf{s}^{\infty}\right],} & \text { if } v=\bar{v} .\end{cases}
$$

Then $p^{\mathrm{f}}\left(\left(l, v, t^{-}\right), p^{\mathrm{S}}\right)$, if bounded by $p^{\mathrm{S}}$, shall first follow $p^{\mathrm{t}}$ and then merge into $p^{\mathrm{S}}$ with a merging segment $\left(p^{\mathrm{t}}\left(t^{\mathrm{m}}\right), \dot{p}^{\mathrm{t}}\left(t^{\mathrm{m}}\right), \underline{a}^{\mathrm{f}}, t^{\mathrm{m}}, t^{+}\right)$. This can be solved analytically with the following FSP algorithm.

FSP-1: Initiate $h=1, t^{+}=t^{\mathrm{m}}=\infty, p^{\mathrm{f}}=\emptyset$ and iterate through the segments in $p^{\mathrm{S}}$ as follows. 
FSP-2: If $v<\bar{v}$, apply the FSO algorithm to solve candidate time points $t^{\mathrm{mc}}:=t^{\mathrm{mf}}\left(\mathrm{s}_{h}^{\mathrm{s}},\left(l, v, \bar{a}^{\mathrm{f}}, t^{-}\right), \underline{a}^{\mathrm{f}}\right)$ and $t^{+\mathrm{c}}:=t^{+\mathrm{f}}\left(\mathbf{s}_{h}^{\mathrm{s}},\left(l, v, \bar{a}^{\mathrm{f}}, t^{-}\right), \underline{a}^{\mathrm{f}}\right)$. If $t^{\mathrm{mc}}>t^{\mathrm{a}}$, revise $t^{\mathrm{mc}}:=\left(t^{\mathrm{mf}_{\mathbf{s}_{h}}},\left(l^{\mathrm{a}}, \bar{v}, 0, t^{\mathrm{a}}\right), \underline{a}^{\mathrm{f}}\right)$ and $t^{+\mathrm{c}}:=$ $t^{+\mathrm{f}}\left(\mathbf{s}_{h}^{\mathrm{S}},\left(l^{\mathrm{a}}, \bar{v}, 0, t^{\mathrm{a}}\right), \underline{a}^{\mathrm{f}}\right)$. If $v=\bar{v}$, solve $t^{\mathrm{mc}}:=t^{\mathrm{mf}}\left(\mathbf{s}_{h}^{\mathrm{S}},\left(l, \bar{v}, 0, t^{-}\right), \underline{a}^{\mathrm{f}}\right)$ and $t^{+\mathrm{c}}:=\left(t^{+\mathrm{f}} \mathbf{s}_{h}^{\mathrm{S}},\left(l, \bar{v}, 0, t^{-}\right), \underline{a}^{\mathrm{f}}\right)$. If $t^{\mathrm{mc}}=-\infty$, the algorithm cannot find a feasible solution and return $p^{\mathrm{f}}\left(\left(l, v, t^{-}\right), p^{\mathrm{S}}\right)=\emptyset, t^{\mathrm{mf}}\left(\left(l, v, t^{-}\right), p^{\mathrm{S}}\right)=$ $-\infty$ and $t^{+\mathrm{f}}\left(\left(p, v, t^{-}\right), p^{\mathrm{S}}\right)=-\infty$. If $t^{+\mathrm{c}} \in\left[t_{h}^{\mathrm{S}}, t_{h+1}^{\mathrm{S}}\right]$, set $t^{\mathrm{m}}=t^{\mathrm{mc}}$ and $t^{+}=t^{+\mathrm{c}}$, and go to the Step FSP-3. Otherwise, $t^{+\mathrm{c}}$ shall be $\infty$. Then if $h<\bar{h}$, set $h=h+1$ and repeat this step.

FSP-3: If $v<\bar{v}$ and $t^{\mathrm{a}}>t^{-}$, append segment $\left(l, v, \bar{a}^{\mathrm{f}}, t^{-}, \min \left(t^{\mathrm{m}}, t^{\mathrm{a}}\right)\right)$ to $p^{\mathrm{f}}$ (appending means adding this segment as the last element of $\left.p^{\mathrm{f}}\right)$. If $t^{\mathrm{m}}>t^{\mathrm{a}}$, append $\left(l^{\mathrm{a}}, \bar{v}, 0, t^{\mathrm{a}}, t^{\mathrm{m}}\right)$ to $p^{\mathrm{f}}$. If $t^{+}<t_{\bar{h}+1}^{\mathrm{S}}$, which indicates $t^{+} \in\left[t_{h}^{\mathrm{S}}, t_{h+1}^{\mathrm{S}}\right]$, we first append segment $\left(l_{h}^{\mathrm{S}}+v_{h}^{\mathrm{S}}\left(t^{+}-t_{h}^{\mathrm{S}}\right)+0.5 a_{h}^{\mathrm{S}}\left(t^{+}-t_{h}^{\mathrm{S}}\right)^{2}, v_{h}^{\mathrm{S}}+a_{h}^{\mathrm{S}}\left(t^{+}-\right.\right.$ $\left.\left.t_{h}^{\mathrm{S}}\right), a_{h}^{\mathrm{S}}, t^{+}, t_{h+1}^{\mathrm{S}}\right)$ to $p^{\mathrm{f}}$, and then append all segments $\left[\mathbf{s}_{h^{\prime}}^{\mathrm{S}}\right]_{h^{\prime}=h+1, \cdots, \bar{h}}$ to $p^{\mathrm{f}}$.

FSP-4: Finally, return $p^{\mathrm{f}}\left(\left(l, v, t^{-}\right), p^{\mathrm{S}}\right)=p^{\mathrm{f}}, t^{\mathrm{mf}}\left(\left(l, v, t^{-}\right), p^{\mathrm{S}}\right)=t^{\mathrm{m}}$ and $t^{+\mathrm{f}}\left(\left(p, v, t^{-}\right), p^{\mathrm{S}}\right)=t^{+}$.

Definition 10. This definition further specifies how we take a symmetric move in the backward shooting, as illustrated in Figure 5(b). Given a quadratic segment $\mathbf{s}^{\prime}:=\left(l^{\prime}, v^{\prime}, a^{\prime}, t^{\prime-}, t^{\prime+}\right)$ with $t^{\prime-}<t^{\prime+}$ (e.g., a segment generated from the forward shooting process), a feasible state point $\left(l, v, t^{-}\right)$with $t^{-}>t^{\prime-}$, acceleration rate $a^{+} \geq 0$ and deceleration rate $a^{-}<0$ (and $a^{-} \leq a^{\prime}$ ), we construct a backward shooting segment $\mathbf{s}:=\left(l, v, a^{+}, t^{-}, t^{\mathrm{m}}\right)$ preceded by a backward merging segment $\mathrm{s}^{\mathrm{m}}:=\left(l^{\mathrm{m}}, v^{\mathrm{m}}, a^{-}, t^{\mathrm{m}}, t^{+}\right)$where again $v^{\mathrm{m}}:=v+a^{+}\left(t^{\mathrm{m}}-t^{-}\right), l^{\mathrm{m}}:=l+v\left(t^{\mathrm{m}}-t^{-}\right)+0.5 a^{+}\left(t^{\mathrm{m}}-t^{-}\right)^{2}$, and $t^{+} \leq t^{\mathrm{m}} \leq t^{-}$, such that condition (8) is satisfied (and thus $\mathbf{s}^{\prime}$ is above $\mathbf{s}$ and $\mathbf{s}^{\mathrm{m}}$ ). And again there are three cases in determining $t^{-}$and $t^{\mathrm{m}}$ values: (I) if no $t^{\mathrm{m}} \in\left(-\infty, t^{-}\right.$can be found to satisfy constraint (8), this shooting operation is infeasible and return $t^{m}=t^{+}=\infty$; (II ) otherwise we try to find $t^{+} \in\left[t^{\prime+}, \min \left\{t^{-}, t^{\prime}\right\}\right]$ and $t^{\mathrm{m}} \in\left[t^{+}, t^{-}\right]$such that $\mathbf{s}^{\prime}$ and $\mathbf{s}^{\mathrm{m}}$ get tangent at time $t^{+}$(as Figure 5(b) indicates); and (III) if no such $t^{+}$is found, set $t^{m}=t^{+}=-\infty$. Solutions $t^{\mathrm{m}}$ and $t^{-}$are denoted as functions $t^{\mathrm{mb}}\left(\mathbf{s}^{\prime},\left(l, v, t^{-}\right), a^{+}, a^{-}\right)$and $t^{-\mathrm{b}}\left(\mathbf{s}^{\prime},\left(l, v, t^{-}\right), a^{+}, a^{-}\right)$, respectively, and they can be solved analytically in the following backward shooting operation (BSO) algorithm.

BSO-1: If $D\left[\mathbf{s}^{\prime}-\left(l, v, a^{-}, t^{-},-\infty\right)\right]<0$, there is no feasible solution, and we just return $t^{\mathrm{m}}=t^{+}=-\infty$ (Case I). Go to Step BSO-3.

BSO-2: Again shift the origin point to $t^{-}$and denote $\hat{t}^{\prime-}:=t^{\prime-}-t^{-}, \hat{t}^{\prime+}:=t^{\prime+}-t^{-}, \hat{t}^{\mathrm{m}}:=t^{\mathrm{m}}-t^{-}$, $\hat{t}^{+}:=t^{+}-t^{-}$and $\hat{t}^{-}:=\min \left\{\hat{t}^{\prime+}, 0\right\}$. And obtain $\mathbf{q}$ by subtracting $\left(l^{\mathrm{m}}, v^{\mathrm{m}}, a^{-}, t^{\mathrm{m}}\right)$ from $\left(l^{\prime}, v^{\prime}, a^{\prime}, t^{\prime-}\right)$, which is formulated the same as that in Step FSO-2.

BSO-2-1: If $a^{\prime}=a^{-}$, test whether $\hat{l}=0$ with $\hat{t}^{\mathrm{m}}=\left(v^{\prime}-v-a^{\prime} \hat{t}^{\prime-}\right) /\left(a^{+}-a^{-}\right)$. If yes and $\hat{t}^{\mathrm{m}} \in\left[\hat{t}^{-}, \hat{t}^{\prime-}\right]$ (Case II),

set $t^{\mathrm{m}}=t^{-}+\hat{t}^{\mathrm{m}}$ and $t^{+}=t^{\prime-}$; Otherwise, return $\hat{t}^{\mathrm{m}}=\hat{t}^{+}=-\infty$ (Case III). Go to BSO-3.

BSO-2-2: If $a^{\prime}>a^{-}$, we need to again solve $\alpha\left(\hat{t}^{\mathrm{m}}\right)^{2}+\beta \hat{t}^{\mathrm{m}}+\gamma=0$ formulated in Step FSO-2-2. In case of $\alpha=\beta=0$ (Case III), set $t^{+}=t^{\mathrm{m}}=-\infty$, and go to Step FSO-3. Otherwise, we again try candidate solutions $\hat{t}^{\mathrm{mc}}$ and $\hat{t}^{+\mathrm{c}}$. In case of $\alpha=0$ but $\beta \neq 0$, solve $\hat{t}^{\mathrm{mc}}=-\gamma / \beta$ and $\hat{t}^{+\mathrm{c}}$ with equation (9). In case of $\alpha \neq 0$, then we solve two sets of solutions $\hat{t}^{\mathrm{mc}}$ and $\hat{t}^{+\mathrm{c}}$ with equations (10) and (9) respectively. if the candidate solutions are not real numbers, then we just set $\hat{t}^{+\mathrm{c}}=\hat{t}^{\mathrm{mc}}=\infty$. Otherwise, try both sets of solutions and select the set satisfying $\hat{t}^{+\mathrm{c}} \leq \hat{t}^{\mathrm{mc}} \leq 0$. With this, if we obtain $\hat{t}^{+\mathrm{C}} \in\left[\hat{t}^{-}, \hat{t}^{\prime-}\right]$ (Case II), we set $t^{\mathrm{m}}=t^{-}+\hat{t}^{\mathrm{mc}}$ and $\hat{t}^{+}=\hat{t}^{+\mathrm{c}}$. Otherwise, set $t^{+}=t^{\mathrm{m}}=\infty$. Go to Step BSO-3.

BSO-3: Finally, we return $t^{\mathrm{mb}}\left(\mathbf{s}^{\prime},\left(l, v, t^{-}, a^{+}\right), a^{-}\right)=t^{m}$ and $t^{+\mathrm{b}}\left(\mathbf{s}^{\prime},\left(l, v, t^{-}, a^{+}\right), a^{-}\right)=t^{+}$.

Definition 11. Symmetric to Definition 9, we extend one backward move BSO to the following backward shooting process (BSP). We consider a backward shooting template trajectory starting at a feasible entry 
state point $\left(l, v, t^{-}\right)$composed by one or both of $\mathbf{s}^{-\mathrm{a}}:=\left(l, v, \bar{a}^{\mathrm{f}}, t^{-}, t^{-\mathrm{a}}:=t^{-}-v / \bar{a}^{\mathrm{b}}\right)$ (which decelerates backward from $v$ to 0 given $v>0)$ and $\mathbf{s}^{-\infty}:=\left(l^{-\mathrm{a}}:=l-0.5 v^{2} / \bar{a}^{\mathrm{b}}, 0,0, t^{-\mathrm{a}},-\infty\right)$ (which denotes the vehicle is stopped prior to time $\left.t^{\text {-a }}\right)$, i.e.,

$$
p^{\mathrm{t}}:= \begin{cases}{\left[\mathbf{s}^{-\infty}, \mathbf{s}^{-\mathrm{a}}\right],} & \text { if } v>0 \\ {\left[\mathbf{s}^{-\infty}\right],} & \text { if } v=0\end{cases}
$$

Further, we are given the original trajectory (e.g., those generated from FSP)

$$
p^{\mathrm{f}}:=\left\{\mathbf{s}_{h}:=\left\{l_{h}, v_{h}, a_{h}, t_{h}, t_{h+1}\right\}\right\}_{h=1, \cdots, \bar{h}} .
$$

We will find a backward shooting trajectory section $p^{\mathrm{b}}\left(\left(l, v, t^{-}\right), p\right)$ to merge into $p^{\mathrm{f}}$ with a merging segment $\left(p^{\mathrm{t}}\left(t^{\mathrm{m}}\right), \dot{p}\left(t^{\mathrm{m}}\right), \underline{a}^{\mathrm{b}}, t^{\mathrm{m}}, t^{+}\right)$and does not exceed (i.e., going left of) $p^{\mathrm{f}}$ at any time. This can be solved analytically with the following BSP algorithm.

BSP-1: Initiate $h$ being the largest segment index of $p^{\mathrm{f}}$ such that $t_{h}<t^{-}$, set $t^{+}=t^{\mathrm{m}}=-\infty, p^{\mathrm{b}}=\emptyset$ and iterate through the segments in $p^{\mathrm{f}}$.

BSP-2: If $v>0$, apply BSO to solve $t^{\mathrm{mc}}:=t^{\mathrm{mb}}\left(\mathbf{s}_{h},\left(l, v, \bar{a}^{\mathrm{b}}, t^{-}\right), \underline{a}^{\mathrm{b}}\right)$ and $t^{+\mathrm{c}}:=t^{\mathrm{mf}}\left(\mathbf{s}_{h},\left(l, v, \bar{a}^{\mathrm{b}}, t^{-}\right), \underline{a}^{\mathrm{b}}\right)$. If $t^{\mathrm{mc}}<t^{-\mathrm{a}}$, revise $t^{\mathrm{mc}}:=t^{\mathrm{mb}}\left(\mathrm{s}_{h},\left(l^{-\mathrm{a}}, 0,0, t^{-\mathrm{a}}\right), \underline{a}^{\mathrm{b}}\right)$ and $t^{+\mathrm{c}}:=t^{+\mathrm{b}}\left(\mathrm{s}_{h},\left(l^{-\mathrm{a}}, 0,0, t^{-\mathrm{a}}\right), \underline{a}^{\mathrm{b}}\right)$ with BSO. If $v=0$, directly solve $t^{\mathrm{mc}}:=t^{\mathrm{mb}}\left(\mathbf{s}_{h},\left(l, 0,0, t^{-}\right), \underline{a}^{\mathrm{b}}\right)$ and $t^{+\mathrm{c}}:=t^{+\mathrm{b}}\left(\mathbf{s}_{h},\left(l, 0,0, t^{-}\right), \underline{a}^{\mathrm{b}}\right)$ with BSO. If $t^{\mathrm{mc}}=\infty$, the algorithm cannot find a feasible solution (because any trajectory through $\left(l, v, t^{-}\right)$will go above $\left.p^{\mathrm{f}}\right)$ and thus returns $p^{\mathrm{b}}\left(\left(l, v, t^{-}\right), p\right)=p^{\mathrm{eb}}\left(\left(l, v, t^{-}\right), p^{\mathrm{f}}\right)=\emptyset$. If $t^{+\mathrm{c}}=-\infty$ and $h=1$, the algorithm cannot find a feasible backward trajectory that can touch $p^{\mathrm{f}}$ and thus return $p^{\mathrm{b}}\left(\left(l, v, t^{-}\right), p\right)=p^{\mathrm{eb}}\left(\left(l, v, t^{-}\right), p^{\mathrm{f}}\right)=\emptyset$. If $t^{+\mathrm{c}} \in\left[t_{h}^{\mathrm{S}}, t_{h+1}^{\mathrm{S}}\right]$, set $t^{\mathrm{m}}=t^{\mathrm{mc}}$ and $t^{+}=t^{+\mathrm{c}}$ and go to Step BSP-3. Otherwise, if $h>1$, set $h=h-1$ and repeat this step.

BSP-3: If $v>0$ and $t^{-\mathrm{a}}<t^{-}$, insert segment $\left(l^{-\mathrm{ma}}, v^{-\mathrm{ma}}, \bar{a}^{\mathrm{b}}, t^{-\mathrm{ma}}, t^{-}\right)$to $p^{\mathrm{b}}$ (inserting means adding this segment before the head of $\left.p^{\mathrm{b}}\right)$, where $t^{-\mathrm{ma}}:=\max \left(t^{\mathrm{m}}, t^{-\mathrm{a}}\right), v^{-\mathrm{ma}}:=v-\bar{a}^{\mathrm{b}}\left(t^{-}-t^{-\mathrm{ma}}\right)$ and $l^{-\mathrm{ma}}:=l-v\left(t^{-}-t^{-\mathrm{ma}}\right)+0.5 \bar{a}^{\mathrm{b}}\left(t^{-}-t^{-\mathrm{ma}}\right)^{2}$. If $t^{\mathrm{m}}<t^{-\mathrm{a}}$, insert $\left(l^{-\mathrm{a}}, 0,0, t^{\mathrm{m}}, t^{-\mathrm{a}}\right)$ to $p^{\mathrm{b}}$. Then we insert merging segment $\left(l^{\mathrm{m}}, v^{\mathrm{m}} \bar{a}^{\mathrm{b}}, t^{+}, t^{\mathrm{m}}\right)$ to $p^{\mathrm{b}}$ where $l^{\mathrm{m}}:=l_{h}+v_{h}\left(t^{+}-t_{h}^{\mathrm{s}}\right)+0.5 a_{h}\left(t^{+}-t_{h}^{\mathrm{S}}\right)^{2}$, and $v^{\mathrm{m}}:=v_{h}+a_{h}\left(t^{+}-t_{h}\right)$.

BSP-4: Now we have obtained a backward shooting trajectory section $p^{\mathrm{b}}\left(\left(l, v, t^{-}\right), p^{\mathrm{f}}\right)=p^{\mathrm{b}}\left(t^{\mathrm{m}}: t^{-}\right)\left(\right.$or $p^{\mathrm{b}}$ for simplicity). We further extend $p^{\mathrm{b}}\left(t^{\mathrm{m}}: t^{-}\right)$by inserting $p^{\mathrm{f}}\left(t_{1}: t^{\mathrm{m}}\right)$ and appending $p^{\mathrm{f}}\left(\left(l, v, t^{-}\right), p^{\mathrm{f}}\right)$ generated from an auxiliary FSP, and construct the extended backward shooting trajectory $p^{\mathrm{eb}}\left(\left(l, v, t^{-}\right), p^{\mathrm{f}}\right)$ $:=\left[p^{\mathrm{f}}\left(t_{1}: t^{\mathrm{m}}\right), p^{\mathrm{b}}\left(t^{\mathrm{m}}: t^{-}\right), p^{\mathrm{f}}\left(\left(l, v, t^{-}\right), p^{\mathrm{f}}\right)\right]$. Return $p^{\mathrm{b}}\left(\left(l, v, t^{-}\right), p^{\mathrm{f}}\right)$ and $p^{\mathrm{eb}}\left(\left(l, v, t^{-}\right), p^{\mathrm{f}}\right)$.

Now we are ready to present the proposed shooting algorithm that yields a trajectory vector $P\left(\bar{a}^{\mathrm{f}}, \underline{a}^{\mathrm{f}}, \bar{a}^{\mathrm{b}}, \bar{a}^{b}\right)$ as a functional of these four acceleration rates.

SH-1: Initialize control variables $\bar{a}^{\mathrm{f}}, \underline{a}^{\mathrm{f}}, \bar{a}^{\mathrm{b}}$ and $\underline{a}^{\mathrm{b}}$. Set $n=1$, trajectory vector $P=\emptyset$. 
SH-2: Apply the FSP to obtain $p_{n}^{\mathrm{f}}=\left[\mathbf{s}_{n k}=\left(l_{n k}, v_{n k}, a_{n k}, t_{n k}, t_{n(k+1)}\right)\right]_{k=1, \cdots, \bar{k}_{n}}=p^{\mathrm{f}}\left(\left(0, v_{n}^{-}, t_{n}^{-}\right), p_{n-1}^{\mathrm{S}}\right)$ (define $p_{0}^{\mathrm{S}}:=\emptyset$ ). We call this process the primary FSP (to differentiate from the auxiliary FSP in the $\mathrm{BSP})$. If $p_{n}^{\mathrm{f}}=\emptyset$, which means that this algorithm cannot find a feasible solution for trajectory platoon $P$, set $P\left(\bar{a}^{\mathrm{f}}, \underline{a}^{\mathrm{f}}, \bar{a}^{\mathrm{b}}, \bar{a}^{b}\right):=P=\emptyset$ and return.

SH-3: This steps checks the need for the BSP. Find the segment index $k_{n}^{L}$ such that $L \in\left[l_{n k^{L}}, l_{n\left(k^{L}+1\right)}\right)$ and solve the time $t_{n}^{L}$ when vehicle $n$ passes location $L$ as follows

$$
t_{n}^{L}:=t_{n k^{L}}+ \begin{cases}\frac{-v_{n k} L}{}+\sqrt{v_{n k}^{2}+2 a_{n k}{ }^{2}\left(L-l_{n k}\right)} & \text { if } a_{n k^{L}} \neq 0 ; \\ \frac{L-l_{n k} L}{v_{n k} L}, & \text { if } a_{n k^{L}}=0 .\end{cases}
$$

If $t_{n}^{L}=G\left(t_{n}^{L}\right)$, which means that $p_{n}^{\mathrm{f}}$ does not violate the exit boundary constraint (3) (or does not run into the red light), we set $p_{n}=p_{n}^{\mathrm{f}}$ and go to SH4. Otherwise, $p_{n}^{\mathrm{f}}$ violates constraint (3) and we need to apply BSP to revise it in the following step. Set $v_{n}^{L}:=v_{n k^{L}}+a_{n k^{L}}\left(t_{n}^{L}-t_{n k^{L}}\right)$, apply the BSP to solve $p_{n}^{\mathrm{b}}=p^{\mathrm{b}}\left(\left(L, v_{n}^{L}, G\left(t_{n}^{L}\right)\right), p_{n}^{\mathrm{f}}\right)$. If the start location of obtain $p_{n}^{\mathrm{b}}$ is no less than 0 , set $p_{n}=p^{\mathrm{eb}}\left(\left(L, v_{n}^{L}, G\left(t_{n}^{L}\right)\right), p_{n}^{\mathrm{f}}\right)$. Otherwise, $p_{n}^{\mathrm{b}}$ can not meet $p_{n}^{\mathrm{f}}$ on this highway segment, and return $P\left(\bar{a}^{\mathrm{f}}, \underline{a}^{\mathrm{f}}, \bar{a}^{\mathrm{b}}, \bar{a}^{b}\right):=\emptyset$.

SH-4: Append $p_{n}$ to $P$. Return $P^{\mathrm{SH}}\left(\underline{a}^{\mathrm{f}}, \bar{a}^{\mathrm{f}}, \underline{a}^{\mathrm{b}}, \bar{a}^{\mathrm{b}}\right):=P$ if $n=N$, or otherwise set $n=n+1$ and go to SH-2.

Although FSO (Definition 8) and BSO (Definition 10) do not explicitly impose speed limits to the generated trajectory segments, as long as a non-empty trajectory vector $P$ is returned by the $\mathrm{SH}$ algorithm, $P$ shall satisfy all constraints defined in Section 2 ( or $P \in \mathcal{P}$ ), as proven in the following proposition.

Proposition 1. If the SH algorithm successfully generates a vector of trajectories $P\left(\underline{a}^{f}, \bar{a}^{f}, \underline{a}^{b}, \bar{a}^{b}\right)$ with $\underline{a}^{f}, \underline{a}^{b} \in[\underline{a}, 0)$ and $\bar{a}^{f}, \bar{a}^{b} \in(0, \bar{a}]$, they shall fall in the feasible trajectory vector set $\mathcal{P}$ defined in $(5)$.

Proof. Since the acceleration of each segment generated from the SH algorithm is either explicitly specified within $[\underline{a}, \bar{a}]$ (i.e., one of $\underline{a}^{\mathrm{f}}, \bar{a}^{\mathrm{f}}, \underline{a}^{\mathrm{b}}, \bar{a}^{\mathrm{b}}$ and 0 ) or just following a shadow trajectory's acceleration that shall fall in $[\underline{a}, \bar{a}]$ as well. So the constraint with respect to acceleration in (1) is satisfied.

Then, we will use mathematical induction to exam the remaining constraints in (1)-(4). First for vehicle 1, FSP can generate $p_{1}$ with at maximum 2 segments, which apparently falls in $\mathcal{T}_{1}^{-}$(and thus both constraints (1)-(2). are satisfied). If BSP is not needed, exit constraint (3) is automatically satisfied and thus $p_{1} \in \mathcal{T}_{1}$. Otherwise, the new segments generated from BSP below $L$ start from a speed no greater than $\bar{v}$, decelerate backwards to a value no less than 0 , and then increase the speed and merge into the forward shooting trajectory at a speed no greater than $\bar{v}$. During this process, the speed shall always stay within $[0, \bar{v}]$ and therefore constraint (1) remains valid. The auxiliary FSP is similar to the primary FSP and thus will not violate constraint (1) as well. Further, the BSP step SH3 does not affect the entry boundary condition (2) and makes exit condition (3) feasible in addition. Therefore we obtain $p_{1} \in \mathcal{T}_{1}$.

Then we assume that $p_{n-1} \in \mathcal{T}_{n-1}, \forall n=2, \cdots, N$, and we will prove that $p_{n} \in \mathcal{T}_{n}\left(p_{n-1}\right)$. If $p_{n}$ is not blocked by $p_{n-1}$ during the FSP, then the construction of $p_{n}$ is similar to that of $p_{1}$ and thus $p_{n}$ should automatically satisfy constraints (1)-(3) and thus $p_{n} \in \mathcal{T}_{n}$. Further, $p_{n}$ shall be always below $p_{n-1}^{\mathrm{s}}$ and therefore $p_{n}$ shall satisfy safety constraint (4), i.e., $p_{n} \in \mathcal{T}_{n}\left(p_{n-1}\right)$. Otherwise, if $p_{n}$ is blocked by $p_{n-1}$, the construction of $p_{n}$ would generate some more segments that merge the forward trajectory into $p_{n-1}^{\mathrm{S}}$ (e.g., as Figure 5(a) illustrates) and then follow $p_{n-1}^{\mathrm{S}}$, as compared with the construction of $p_{1}$. Due to the induction assumption, the segments following $p_{n-1}^{\mathrm{S}}$ shall satisfy kinematic constraint (1) the same as the corresponding segments in $p_{n-1}$. For the merging segment, since it starts from a forward shooting segment and ends at a 
shadow segment and therefore its speed range should be bounded by $[0, \bar{v}]$. Therefore, we obtain $p_{n} \in \mathcal{T}$. Again, the primary FSP ensures that $p_{n}$ satisfies the entry boundary (2) and BSP ensures that $p_{n}$ satisfy exit constraint (3). This yields $p_{n} \in \mathcal{T}_{n}$. Further we see that any segment generated from FSP shall be either below or on $p_{n-1}^{\mathrm{S}}$. If the BSP generates new segments, they shall be strictly right to the forward shooting trajectory. Therefore, $p_{n}$ shall fall in $\mathcal{T}_{n}\left(p_{n-1}\right)$. This proves that $P\left(\underline{a}^{\mathrm{f}}, \bar{a}^{\mathrm{f}}, \underline{a}^{\mathrm{b}}, \bar{a}^{\mathrm{b}}\right) \in \mathcal{P}$.

\subsection{Shooting Heuristic for the Lead Vehicle Problem}

The above proposed SH algorithm can be easily adapted to solve LVP (6). The only modifications are to fix $p_{1}$ and to set $\mathcal{G}=[-\infty, \infty]$ (and therefore no BSP is needed). We denote this adapted SH for LVP by SHL. More importantly, we further find that SHL can be alliteratively implemented in a parallel manner. Each trajectory can be calculated directly from the input parameters without its preceding trajectory's information. We denote this parallel alternative of SHL with PSHL. Apparently, PSHL allows further improved computational efficiency with parallel computing. This section describes PSHL and validates that PSHL indeed solves LVP. We first introduce an extended forward shooting operation (EFSO) that merges two feasible trajectories.

EFSO-1: Given two feasible trajectories $p:=\left[\mathbf{s}_{j}:=\left(l_{j}, v_{j}, a_{j}, t_{j}, t_{j+1}\right)\right]_{j=1,2, \cdots, J}$ and $p^{\prime}=\mathbf{s}_{k}^{\prime}:=\left[\left(l_{k}^{\prime}, v_{k}^{\prime}, a_{k}^{\prime}\right.\right.$, $\left.\left.t_{k}^{\prime}, t_{k+1}^{\prime}\right)\right]_{k=1,2, \cdots, K} \in \mathcal{T}$, and deceleration rate $a^{-}<0$. Set iterators $j=1$ and $k=1$.

EFSO-2: Solve $t_{j k}^{\mathrm{m}}:=t^{\mathrm{mf}}\left(\mathbf{s}_{k}^{\prime},\left(l_{j}, v_{j}, t_{j}, a_{j}\right), a^{-}\right)$and $t_{j k}^{+}=t^{+\mathrm{f}}\left(\mathbf{s}_{k}^{\prime},\left(l_{j}, v_{j}, t_{j}, a_{j}\right), a^{-}\right)$. If $t_{j k}^{\mathrm{m}}=-\infty$, return $t^{\mathrm{m}}\left(p^{\prime}, p, a^{-}\right)=t^{+}\left(p^{\prime}, p, a^{-}\right)=-\infty$. If $t_{j k}^{\mathrm{m}}<\infty$ and $t_{j k}^{\mathrm{m}} \in\left[t_{j}, t_{j+1}\right]$, return $t^{\mathrm{m}}\left(p^{\prime}, p, a^{-}\right)=t_{j k}^{\mathrm{m}}$, $t^{+}\left(p^{\prime}, p, a^{-}\right)=t_{j k}^{+}$. If $k<K$, set $k=k+1$ and repeat this step; otherwise, go to the next step.

EFSO-2: If $j<J$, set $j=j+1$ and $k=1$ and go to Step EFSO-2. Otherwise, return $t^{\mathrm{m}}\left(p^{\prime}, p, a^{-}\right)=$ $t^{+}\left(p^{\prime}, p, a^{-}\right)=\infty$.

Based on EFSO, we devise the following extended forward shooting process (EFSP) that generates a forward shooting trajectory constrained by a series of upper bound trajectories.

EFSP-1: Given a feasible state point $(l, v, t)$, and a set of trajectories $\left\{p_{m} \in \mathcal{T}\right\}_{m=1, \cdots, M}$. Initiate $m=1$ and $p=p^{\mathrm{f}}((l, v, t), \emptyset)$ with FSP.

EFSP-2: Call EFSO to solve $t^{\mathrm{m}}:=t^{\mathrm{m}}\left(p_{m}, p, \underline{a}^{\mathrm{f}}\right)$ and $t^{+}:=t^{+}\left(p_{m}, p, \underline{a}^{\mathrm{f}}\right)$. If $t^{\mathrm{m}}=-\infty$, return $p^{\mathrm{f}}\left((l, v, t),\left\{p_{m}\right\}_{m=1, \cdots, M}\right)=\emptyset$. If $t^{\mathrm{m}}<\infty$, revise $p:=\left[p\left(t^{-}(p): t^{\mathrm{m}}\right),\left(p\left(t^{\text {mincluding their solution feasibility and } \mathrm{r}}\right.\right.\right.$ $\left.p_{m}\left(t^{+}: \infty\right)\right]$.

EFSP-3: If $m<M$, set $m=m+1$ and go to PSFP-2; otherwise return $p^{\mathrm{f}}\left((l, v, t),\left\{p_{m}\right\}_{m=1, \cdots, M}\right)=p$.

Then we are ready to present the PSHL algorithm as follows.

PSHL-1: Given lead trajectory $p_{1} \in \mathcal{T}$ and boundary condition $\left[v_{n}^{-}, t_{n}^{-}\right]_{n \in \mathcal{N} \backslash\{1\}}$. Initialize acceleration parameters $\bar{a}^{\mathrm{f}}$ and $\underline{a}^{\mathrm{f}}$. Set $\bar{p}_{1}:=p_{1}, \bar{p}_{n}:=p^{\mathrm{f}}\left(\left(0, v_{n}^{-}, t_{n}^{-}\right), \emptyset\right), \forall n \in \mathcal{N} \backslash\{1\}$. Set initial trajectory platoon $P=\left[p_{1}\right], n=2$.

PSHL-2: Solve $p_{n}:=p^{\mathrm{f}}\left(\left(0, v_{n}^{-}, t_{n}^{-}\right),\left\{\bar{p}_{m}^{\mathrm{S}^{n-m}}\right\}_{m=1, \cdots, n-1}\right)$ with EFSP.

PSHL-3: If $p_{n}=\emptyset$, return $P^{\mathrm{PSH}}\left(\underline{a}^{\mathrm{f}}, \bar{a}^{\mathrm{f}}\right):=\emptyset$. Otherwise, append $p_{n}$ to $P$. If $n=N$, return $P^{\mathrm{PSH}}\left(\underline{a}^{\mathrm{f}}, \bar{a}^{\mathrm{f}}\right):=$ $P$; otherwise, set $n=n+1$ and go to PSHL-2. 
Proposition 2. $P^{P S H}\left(\underline{a}^{f}, \bar{a}^{f}\right)$ obtained from $P S H L$ is identical to $P^{\mathrm{SH}}\left(\underline{a}^{\mathrm{f}}, \bar{a}^{\mathrm{f}}, \underline{a}^{\mathrm{b}}, \bar{a}^{\mathrm{b}}\right)$ when $p_{1} \in \mathcal{T}$ is fixed and $\mathcal{G}=(-\infty, \infty)$.

Proof. We first consider the cases where $P^{\mathrm{PSH}}\left(\underline{a}^{\mathrm{f}}, \bar{a}^{\mathrm{f}}\right) \neq \emptyset$. We will prove this proposition via induction with the iterator being vehicle index $n$. Let $\left[p_{1}, p_{2}, \cdots, p_{N}\right]$ denote the trajectories in $P^{\mathrm{PSH}}\left(\underline{a}^{\mathrm{f}}, \bar{a}^{\mathrm{f}}\right)$ and $\left[p_{1}^{\prime}, p_{2}^{\prime}, \cdots, p_{N}^{\prime}\right]$ denote the trajectories in $P^{\mathrm{SH}}\left(\underline{a}^{\mathrm{f}}, \bar{a}^{\mathrm{f}}, \underline{a}^{\mathrm{b}}, \bar{a}^{\mathrm{b}}\right)$. Apparently, when $n=1, p_{1}^{\prime}=p_{1}$ since the lead vehicle's trajectory is fixed. Assume that when $n=k, p_{k}^{\prime}=p_{k}$. Based on the definition of SH, $p_{k+1}^{\prime}=\left[\bar{p}_{k+1}\left(t_{k+1}^{-}: t^{\mathrm{m}}\right),\left(\bar{p}_{k+1}\left(t^{\mathrm{m}}\right), \dot{\bar{p}}_{k+1}\left(t^{\mathrm{m}}\right), \underline{a}^{\mathrm{f}}, t^{\mathrm{m}}, t^{+}\right), p_{k}^{\prime \mathrm{s}}\left(t^{+}: \infty\right)\right]$ where $\bar{p}_{k+1}:=$ $p^{\mathrm{f}}\left(\left(0, v_{k+1}^{-}, t_{k+1}^{-}\right), \emptyset\right), p_{k}^{\prime \mathrm{S}}(t):=p_{k}^{\prime}(t-\tau)-s, t^{\mathrm{m}}:=t^{\mathrm{m}}\left(p_{k}^{\prime \mathrm{s}}, \bar{p}_{k+1}, \underline{a}^{\mathrm{f}}\right), t^{+}:=t^{+}\left(p_{k}^{\prime \mathrm{s}}, \bar{p}_{k+1}, \underline{a}^{\mathrm{f}}\right)$. Based on the induction assumption, $p_{k}^{\mathrm{S}}=p_{k}^{\mathrm{S}}$ can be obtained by repeatedly calling EFSO to merge $\left\{\bar{p}_{m}^{\mathrm{S}^{k+1-m}}\right\}_{m=1, \cdots, k}$ as in PSHL-2. Therefore, $p_{k+1}^{\prime}$ is obtained by merging $\left\{\bar{p}_{m}^{\mathrm{s}^{k+1-m}}\right\}_{m=1, \cdots, k+1}$ and thus $p_{k+1}^{\prime}=p_{k+1}$.

When $P^{\mathrm{PSH}}\left(\underline{a}^{\mathrm{f}}, \bar{a}^{\mathrm{f}}\right)=\emptyset$, from the above discussion that shows the equivalence of generating $p_{k}$ and $p_{k}^{\prime}$, it is obvious that $P^{\mathrm{SH}}\left(\underline{a}^{\mathrm{f}}, \bar{a}^{\mathrm{f}}\right)=\emptyset$ as well. This completes the proof.

Corollary 1. Given lead trajectory $p_{1} \in \mathcal{T}, P S H L$ yields $P^{P S H}\left(\underline{a}^{f}, \bar{a}^{f}\right) \in \mathcal{P}^{L V P}\left(p_{1}\right)$ if $\underline{a}^{f}, \bar{a} f \in[\underline{a}, \bar{a}]$.

\section{Theoretical Property Analysis}

This section analyzes theoretical properties of the proposed SH algorithms, including their solution feasibility and relationships with the classic traffic flow theory. It is actually challenging to analyze such properties because the original problem defined in Section 2 involves infinite-dimensional trajectory variables and highly nonlinear constraints. Fortunately, the concept of time geography (Miller, 2005) is found related to the bounds to feasible trajectory ranges. This concept has been used to investigate macroscopic transportation network problems (Tang et al., 2015; Tong et al., 2015) but seldom applied to microscopic traffic flow. We generalize this concept to enable the following theoretical analysis.

\subsection{Quadratic Time Geography}

Following the idea of Kuijpers et al. (2011), we first generalize the time geography theory considering acceleration range $[\bar{a}, \underline{a}]$ in additional to speed range $[0, \bar{v}]$. These generalized theory, referred to as the quadratic time geography (QTG) theory, are illustrated in Figure 6 and discussed in detail in this subsection.

Definition 12. We call the set of feasible trajectories (i.e., in $\mathcal{T}$ ) passing a common feasible state point $\left(l, v, t^{-}\right)$the quadratic cone of $\left(l, v, t^{-}\right)$, denoted by $\mathcal{C}_{l v t^{-}}$, illustrated as the shaded area in Figure 6(a) and formulated below:

$$
\mathcal{C}_{l v t^{-}}=\left\{p \mid p \in \mathcal{T}, p\left(t^{-}\right)=l, \dot{p}\left(t^{-}\right)=v, \forall t \in(-\infty, \infty)\right\},
$$

where the upper bound trajectory $\bar{p}_{l v t^{-}}$of $\left(l, v, t^{-}\right)$, illustrated as the top boundary of the shade in Figure $6(\mathrm{a})$, is formulated as

$$
\bar{p}_{l v t^{-}}(t):= \begin{cases}l-\frac{v^{2}}{2 \bar{a}}, & \text { if } t \in\left(-\infty, t^{-}-\frac{v}{\bar{a}}\right] ; \\ l+v\left(t-t^{-}\right)+0.5 \bar{a}\left(t-t^{-}\right)^{2}, & \text { if } t \in\left[t^{-}-\frac{v}{\bar{a}}, t^{-}+\frac{\bar{v}-v}{\bar{a}}\right] ; \\ l+\bar{v}\left(t-t^{-}\right)-\frac{(\bar{v}-v)^{2}}{2 \bar{a}}, & \text { if } t \in\left[t^{-}+\frac{\bar{v}-v}{\bar{a}}, \infty\right),\end{cases}
$$

and the lower bound trajectory $\underline{p}_{l v t^{-}}$of $\left(l, v, t^{-}\right)$, illustrated as the bottom boundary of the shade in Figure 6(a), is formulated as 


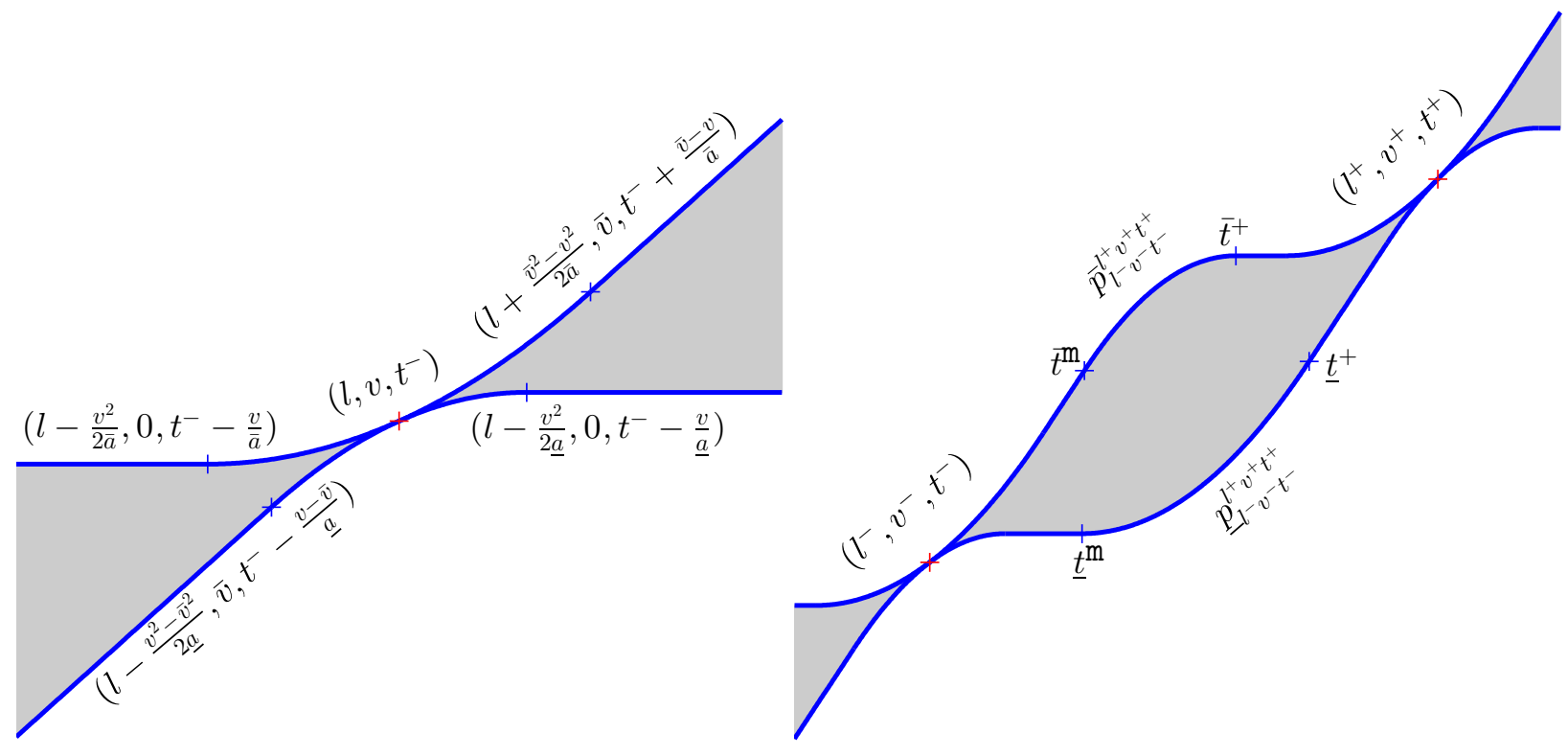

(a)

(b)

Figure 6: Illustrations of the generalized time geography theory in : (a) quadratic cone; and (b) quadratic prism.

$$
\underline{p}_{l v t^{-}}(t)= \begin{cases}l+\bar{v}\left(t-t^{-}\right)-\frac{(\bar{v}-v)^{2}}{2 \underline{a}}, & \text { if } t \in\left(-\infty, t^{-}-\frac{v-\bar{v}}{\underline{a}}\right] ; \\ l+v\left(t-t^{-}\right)+0.5 \underline{a}\left(t-t^{-}\right)^{2}, & \text { if } t \in\left[t^{-}-\frac{v-\bar{v}}{\underline{a}}, t^{-}+\frac{-v}{\underline{a}}\right] ; \\ l+\frac{-v^{2}}{2 \underline{a}}, & \text { if } t \in\left[t^{-}+\frac{-v}{\underline{a}}, \infty\right) .\end{cases}
$$

In other words, $\bar{p}_{l v t^{-}}$is composed of quadratic segments $\left(l-\frac{v^{2}}{2 \bar{a}}, 0,0, t^{-}-\frac{v}{\bar{a}},-\infty\right),\left(l-\frac{v^{2}}{2 \bar{a}}, 0, \bar{a}, t^{-}-\frac{v}{\bar{a}}, t^{-}+\frac{\bar{v}-v}{\bar{a}}\right)$ and $\left(l+\frac{\bar{v}^{2}-v^{2}}{2 \bar{a}}, \bar{v}, 0, t^{-}+\frac{\bar{v}-v}{\bar{a}}, \infty\right)$, and $\bar{p}_{l v t^{-}}$is comprised of quadratic segments $\left(l-\frac{v^{2}-\bar{v}^{2}}{2 \underline{a}}, \bar{v}, 0, t^{-}-\frac{v-\bar{v}}{\underline{a}},-\infty\right)$, $\left(l-\frac{v^{2}-\bar{v}^{2}}{2 \underline{a}}, \bar{v}, \bar{a}, t^{-}-\frac{v-\bar{v}}{\underline{a}}, t^{-}+\frac{-v}{\underline{a}}\right)$ and $\left(l+\frac{-v^{2}}{2 \underline{a}}, 0,0, t^{-}+\frac{-v}{\underline{a}}, \infty\right)$. Note that $\bar{p}_{l v t^{-}}=p^{\mathrm{f}}\left(\left(l, v, t^{-}\right), \emptyset\right)$ from the FSP with $\bar{a}^{\mathrm{f}}=\bar{a}$ and $\underline{a}^{\mathrm{f}}=\underline{a}, \bar{p}_{l v t^{-}}\left(t^{-}\right)=\underline{p}_{l v t^{-}}\left(t^{-}\right)=l$, and $\bar{p}_{l v t^{-}}(t) \geq \underline{p}_{l v t^{-}}(t), \forall t \neq t^{-}$. Further, $\mathcal{C}_{l v t^{-}}$ is always non-empty as long as $\left(l, v, t^{-}\right)$is feasible.

Definition 13. We call the set of trajectories in $\mathcal{T}$ passing two feasible state points $\left(l^{-}, v^{-}, t^{-}\right)$and $\left(l^{+}, v^{+}, t^{+}\right)$with $t^{-}<t^{+}, l^{-} \leq l^{+}$a quadratic prism, denoted by $\mathcal{P}_{l^{-} v^{-} t^{+}}^{l^{+}}$, as illustrated in Figure 6(b) and formulated below:

$$
\mathcal{P}_{l^{-} v^{-} t^{+} t^{+}}^{l^{-}}:=\left\{p \in \mathcal{T} \mid p\left(t^{-}\right)=l^{-}, \dot{p}\left(t^{-}\right)=v^{-}, p\left(t^{+}\right)=l^{+}, \dot{p}\left(t^{+}\right)=v^{+}\right\}=\mathcal{C}^{p^{-} v^{-} t^{-}} \bigcap \mathcal{C}^{p^{+} v^{+} t^{+}} .
$$

The upper bound of this $\mathcal{P}_{l^{-} v^{-} t^{+}}^{l^{+}}$(as the top boundary of the shade in 6(b)), denoted by $\bar{p}_{l^{-} v^{+} t^{+} t^{+}}$, should be composed by $\bar{p}_{l^{-} v^{-} t^{-}}\left(\infty: \bar{t}^{\mathrm{m}}\right)$, merging segment $\overline{\mathbf{s}}^{\mathrm{m}}\left(\bar{p}_{l^{-} v^{-} t^{-}}\left(\bar{t}^{\mathrm{m}}\right), \dot{\bar{p}}_{l^{-} v^{-} t^{-}}\left(\bar{t}^{\mathrm{m}}\right), \bar{a}, \bar{t}^{\mathrm{m}}, \bar{t}^{+}\right)$and $\bar{p}_{l^{+} v^{+} t^{+}}\left(\bar{t}^{+}: \infty\right)$, where we can actually apply FSP with $\bar{a}^{\mathrm{f}}=\bar{a}$ and $\underline{a}^{\mathrm{f}}=\underline{a}$ to obtain connection points $\bar{t}^{\mathrm{m}}:=t^{\mathrm{mf}}\left(\left(l^{-}, v^{-}, t^{-}\right), \bar{p}_{l^{+} v^{+} t^{+}}\right)$and $\bar{t}^{+}:=t^{+\mathrm{f}}\left(\left(l^{-}, v^{-}, t^{-}\right), \bar{p}_{l^{+} v^{+} t^{+}}\right)$. The lower bound of this prism (as the bottom boundary of the shade in $6(\mathrm{~b}))$, denoted by $\underline{p}_{l^{-} v^{+} t^{+}}^{l^{+}}$, is composed by section $\underline{p}_{l^{-} v^{-} t^{-}}\left(\infty: \underline{t}^{\mathrm{m}}\right)$, merging segment $\overline{\mathrm{s}}^{\mathrm{m}}\left(\underline{p}_{l^{-} v^{-} t^{-}}\left(\underline{t}^{\mathrm{m}}\right), \underline{\dot{p}}_{l^{-} v^{-} t^{-}}\left(\underline{t}^{\mathrm{m}}\right), \bar{a}, \underline{t}^{\mathrm{m}}, \underline{t}^{+}\right)$and $\underline{p}_{l^{+} v^{+} t^{+}}\left(\underline{t}^{+}: \infty\right)$, where we can apply FSP in a transformed coordinate system to solve $\underline{t}^{\mathrm{m}}$ and $\underline{t}^{+}$. We shift the first shift the origin to $\left(l^{+}, t^{+}\right)$ 
and rotate the whole coordinate system by 180 degree. Then state points $\left(l^{+}, v^{+}, t^{+}\right)$and $\left(l^{-}, v^{-}, t^{-}\right)$ transfer into $\left(0, v^{+}, 0\right)$ and $\left(l^{+}-l^{-}, v^{-}, t^{+}-t^{-}\right)$, respectively, and $\underline{p}_{l^{-} v^{-} t^{-}}$transfers into $\underline{\hat{p}}_{l^{-} v^{-} t^{-}}$defined as $\underline{\hat{p}}_{l^{-} v^{-} t^{-}}(t):=l^{+}-\underline{p}_{l^{-} v^{-} t^{-}}\left(2 t^{+}-\left(t^{-}+t\right)\right)$. Then we solve $\underline{\hat{t}}^{\mathrm{m}}:=t^{\mathrm{mf}}\left(\left(0, v^{+}, 0\right), \underline{\hat{p}}_{l^{-} v^{-} t^{-}}\right)$and $\underline{\hat{t}}^{+}:=t^{+\mathrm{f}}\left(\left(0, v^{+}, 0\right), \underline{\hat{p}}_{l^{-} v^{-} t^{-}}\right)$with $\mathrm{FSP}$ with $a^{\mathrm{f}}=-\underline{a}$ and $\underline{a}^{\mathrm{f}}=-\bar{a}$. Then we obtain $\underline{t}^{+}=t^{+}-\underline{\hat{t}}^{\mathrm{m}}$ and $\underline{t}^{\mathrm{m}}=t^{+}-\underline{\hat{t}}^{+}$.

Note that the feasibility of $\mathcal{P}_{l^{-} v^{+} t^{+} t^{+}}$depends on the values of $\left(l^{-}, v^{-}, t^{-}\right)$and $\left(l^{+}, v^{+}, t^{+}\right)$, as discussed in the following propositions.

Proposition 3. Given two feasible state points $\left(l^{-}, v^{-}, t^{-}\right)$and $\left(l^{+}, v^{+}, t^{+}\right)$, quadratic cone $\mathcal{P}_{l^{-} v^{-} t^{+}}^{t^{+}}$is not empty if and only if $D\left(\bar{p}_{l^{+} v^{+} t^{+}}-\underline{p}_{l^{-} v^{-} t^{-}}\right) \geq 0$ and $D\left(\bar{p}_{l^{-} v^{-} t^{-}}-\underline{p}_{l^{+} v^{+} t^{+}}\right) \geq 0$.

Proof. We first prove the necessity. If there exists a feasible trajectory $p \in \mathcal{P}_{l^{-} v^{-} t^{+}}^{l^{+}}$, then we know the

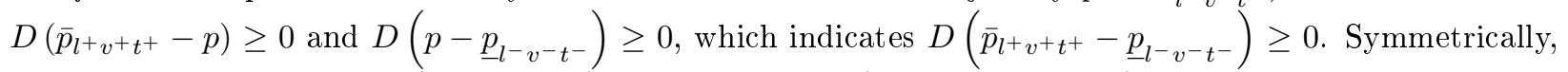
$D\left(\bar{p}_{l^{-} v^{-} t^{-}}-p\right) \geq 0$ and $D\left(p-\underline{p}_{l^{+} v^{+} t^{+}}\right) \geq 0$ indicates $D\left(\bar{p}_{l^{-} v^{-} t^{-}}-\underline{p}_{l^{+} v^{+} t^{+}}\right) \geq 0$.

Then we investigate the sufficiency. Given $D\left(\bar{p}_{l^{+} v^{+} t^{+}}-\underline{p} \underline{l}^{-} v^{-} t^{-}\right) \geq 0$ and $\left.D\left(\bar{p}_{l^{-} v^{-} t^{-}}\right) \underline{p}_{l^{+} v^{+} t^{+}}\right) \geq$ 0 , we can first obtain that $l^{-}<l^{+}$and $t^{-}<t^{+}$. Further we know that there exists a point $\bar{t}^{*} \in$ $\left[t^{-}, t^{+}\right]$such that $\bar{p}_{l^{+} v^{+} t^{+}}(t) \geq \bar{p}_{l^{-} v^{-} t^{-}}(t), \forall t \in\left[-\infty, \bar{t}^{*}\right], \bar{p}_{l^{+} v^{+} t^{+}}\left(\bar{t}^{*}\right)=\bar{p}_{l^{-} v^{-} t^{-}}\left(\bar{t}^{*}\right)$ and $\bar{p}_{l^{+} v^{+} t^{+}}(t) \leq$ $\bar{p}_{l^{-} v^{-} t^{-}}(t), \forall t \in\left[\bar{t}^{*}, \infty\right]$. Then we can obtain a trajectory $p^{\mathrm{m}} \in \mathcal{C}_{l^{-} v^{-} t^{-}}$composed by $\bar{p}_{l v t^{-}}\left(t^{-}: \hat{t}^{\mathrm{m}}\right), \mathbf{s}^{\mathrm{m}}:=$ $\left(\bar{p}_{l v t^{-}}\left(\hat{t}^{\mathrm{m}}\right), \dot{\bar{p}}_{l v t^{-}}\left(\hat{t}^{\mathrm{m}}\right), \underline{a}, \hat{t}^{\mathrm{m}}, \bar{t}^{\mathrm{m}}\right), \bar{p}_{l^{+} v^{+} t^{+}}\left(\bar{t}^{\mathrm{m}}: \infty\right)$ satisfying $t^{-} \leq \hat{t}^{\mathrm{m}} \leq \bar{t}^{\mathrm{m}}<\infty$ and $D\left(\bar{p}_{l^{+} v^{+} t^{+}}-p^{\mathrm{m}}\right) \geq$ 0 . Next, we will prove $\bar{t}^{\mathrm{m}} \leq t^{+}$by contradiction. If $\bar{t}^{\mathrm{m}}>t^{+}$, then $\bar{p}_{l^{+} v^{+} t^{+}}\left(\bar{t}^{\mathrm{m}}\right)>l^{+}$and $\dot{\bar{p}}_{l^{+} v^{+} t^{+}}\left(\bar{t}^{\mathrm{m}}\right)>$ $v^{+}$. Since segment $\mathbf{s}^{\mathrm{m}}$ decelerates at $\underline{a}$, then $D\left(\underline{p}_{l^{+} v^{+} t^{+}}-\mathbf{s}^{\mathrm{m}}\right)>0$, which however is contradictory to $D\left(\bar{p}_{l^{-} v^{-} t^{-}}-\underline{p}_{l^{+} v^{+} t^{+}}\right) \geq 0$ because the start point of $\mathrm{s}^{\mathrm{m}}$ is on $\bar{p}_{l^{-} v^{-} t^{-}}$. This proves that $t^{-} \leq \hat{t}^{\mathrm{m}} \leq \bar{t}^{\mathrm{m}} \leq t^{+}$. Therefore, $p^{\mathrm{m}} \in \mathcal{C}_{l^{-} v^{-} t^{-}} \cap \mathcal{C}_{l^{+} v^{+} t^{+}}=\mathcal{P}_{l^{-} v^{-} t^{+}}$. This completes the proof.

Proposition 4. Given any $\delta \geq 0$ and two feasible state points $\left(l^{-}, v^{-}, t^{-}\right)$and $\left(l^{+}, v^{+}, t^{+}\right)$with $D\left(\bar{p}_{l^{-}, v^{-}, t^{-}}\right.$ $\left.\underline{p}_{l^{+}, v^{+}, t^{+}}\right) \geq 0$, if quadratic prism $\mathcal{P}_{l^{-} v^{-} t^{-}}^{l^{+} v^{+}\left(t^{+}+\delta\right)}$ is not empty, then $\mathcal{P}_{l^{-} v^{-} t^{+}}^{l^{+} t^{+}}$is not empty and $D\left(\bar{p}_{l^{-} v^{-} t^{-}}^{l^{+} v^{+}}-p\right) \geq$ $0, \forall p \in \mathcal{P}_{l^{-} v^{-} t^{-}}^{l^{+} v^{+}\left(t^{+}+\delta\right)}$.

Proof. If $\mathcal{P}_{l^{-} v^{-} t^{-}}^{\left.l^{+} t^{+}+\delta\right)}$ is not empty, Proposition 3 indicates that $D\left(\bar{p}_{l^{+} v^{+}\left(t^{+}+\delta\right)}-\underline{p}_{l^{-} v^{-} t^{-}}\right) \geq 0$. Further, apparently $D\left(\bar{p}_{l+v^{+} t^{+}}-\bar{p}_{l^{+} v^{+}\left(t^{+}+\delta\right)}\right) \geq 0$ and thus due to the transitive property of function $D$, we obtain $D\left(\bar{p}_{l^{+} v^{+} t^{+}}-\underline{p}_{l^{-} v^{-} t^{-}}\right) \geq 0$, which combined with the given condition $D\left(\bar{p}_{l^{-}, v^{-}, t^{-}}-\underline{p}_{l^{+}, v^{+}, t^{+}}\right) \geq 0$ indicates that $\mathcal{P}_{l^{-} v^{+} v^{+} t^{+}}$is not empty based on Proposition 3 .

Further, for any $p \in \mathcal{P}_{l^{-} v^{-} t^{-}}^{l^{+} v^{+}\left(t^{+}+\delta\right)}$, we have $D\left(\bar{p}_{l^{-} v^{-} t^{-}}^{l^{+} v^{+}\left(t^{+}+\delta\right)}-p\right)>0$. Since $D\left(\bar{p}_{l^{+} v^{+} t^{+}}-\bar{p}_{l^{+} v^{+}\left(t^{+}+\delta\right)}\right) \geq$ 0 , we also have $D\left(\bar{p}_{l^{+} v^{+} t^{+}}-p\right) \geq 0$ due to the transitive property of function $D$. This implies that $p \in$ $\mathcal{P}_{l^{-} v^{-} t^{-}}^{l^{+}} t^{+}$and thus $D\left(\bar{p}_{l^{-} v^{-} t^{+}}^{+}-p\right) \geq 0$. This completes the proof.

Remark 4. Note that as $\bar{a} \rightarrow \infty$ and $\underline{a} \rightarrow-\infty$, every smooth speed transition segment on the borders of a quartic cone or prism reduces into a vertex, and the QTG concept converges to the classic time geography (Miller, 2005). Besides, when the spatiotemporal range of the studied problem is far greater than that where acceleration $\bar{a}$ and deceleration $\underline{a}$ is discernible, neither is QTG much different from the classic time geography. 


\subsection{Relationship Between QTG and SH}

As preparing for the investigation of the feasibility and optimality of the SH solution, we now discuss the relationships between trajectories generated from FSP and BSP and the borders of the corresponding quadratic cones and prisms. For uniformity, this subsection only considers FSP and BSP with the extreme acceleration control variable values, i.e., $\left[\bar{a}^{\mathrm{f}}, \underline{a}^{\mathrm{f}}, \bar{a}^{\mathrm{b}}, \underline{a}^{\mathrm{b}}\right]=[\bar{a}, \underline{a}, \bar{a}, \underline{a}]$.

Proposition 5. The forward shooting trajectory $p^{f}\left(\left(l, v, t^{-}\right), \emptyset\right)$ generated from FSP overlaps $\bar{p}_{l v t^{-}}\left(t^{-}: \infty\right)$.

Proposition 6. Given two feasible state points $\left(l^{-}, v^{-}, t^{-}\right)$and $\left(l^{+}, v^{+}, t^{+}\right)$with $l^{+}>l^{-}$and $t^{+}>t^{-}$such that quadratic prism $\mathcal{P}_{l^{-} v^{+} t^{+} t^{+}} \neq \emptyset$, the extended backward shooting trajectory $p^{e b}\left(\left(l^{+}, v^{+}, t^{+}\right), p^{f}\left(\left(l^{-}, v^{-}, t^{-}\right), \emptyset\right)\right)$ generated from BSP overlaps $\bar{p}_{l^{-} v^{+} t^{+}} t^{+}\left(t^{-}: \infty\right)$.

These two propositions obviously hold based on the definitions of FSP and BSP and thus we omit the proofs. These properties can be extended to cases where the current trajectory is bounded by one or multiple preceding trajectories from the top.

Definition 14. Given a set of trajectories $\mathbf{q}=\left\{q_{1}, q_{2}, \cdots, q_{M} \in \mathcal{T}\right\}$, we define $u(\mathbf{q}, t):=\min _{m \in\{1, \cdots, M\}} q_{m}(t), \forall t$ and we call function $u(\mathbf{q}, \cdot)$ a quasi-trajectory and denote it with $u(\mathbf{q})$ for simplicity. Let $\mathcal{U}$ denote the set of all quasi-trajectories. Note that distance function $D$ can be easily extended to $\mathcal{U}$, i.e., $D\left(u-u^{\prime}\right):=$ $\min _{t \in(\infty, \infty)}\left(u(t)-u^{\prime}(t)\right), \forall u, u^{\prime} \in \mathcal{U}$. We can also denote EFSP result $p^{\mathrm{f}}((l, v, t), \mathbf{q})$ as $p^{\mathrm{f}}((l, v, t), u(\mathbf{q}))$. Further, let $p(\mathbf{q})$ denote the trajectory generated by merging all trajectories in $\mathbf{q}$ with EFSO, and we can also denote BSP result $p^{\mathrm{b}}((l, v, t), p(\mathbf{q}))$ and $p^{\mathrm{eb}}((l, v, t), p(\mathbf{q}))$ as $p^{\mathrm{b}}((l, v, t), u(\mathbf{q}))$ and $p^{\mathrm{eb}}((l, v, t), u(\mathbf{q}))$, respectively, for simplicity.

Definition 15. Given a feasible state point $\left(l, v, t^{-}\right)$and a quasi-trajectory $u \in \mathcal{U}$, we define $\mathcal{C}_{l v t^{-}}^{u}:=$ $\left\{p \mid p \in \mathcal{C}_{l v t^{-}}, D(u-p) \geq 0\right\}$, which we call a bounded cone of $\left(l, v, t^{-}\right)$with respect to $u$.

Definition 16. Given two feasible state points $\left(l^{-}, v^{-}, t^{-}\right),\left(l^{+}, v^{+}, t^{+}\right)$and a quasi-trajectory $u \in \mathcal{U}$, we define $\mathcal{P}_{l^{-} v^{-} t^{-}}^{l^{+} v^{+}}:=\left\{p \mid p \in \mathcal{P}_{l^{-} v^{-} t^{+}}^{l^{+} t^{+}}, D(u-p) \geq 0\right\}$, which we call a bounded prism of $\left(l^{-}, v^{-}, t^{-}\right)$and $\left(l^{+}, v^{+}, t^{+}\right)$with respect to $u$.

Apparently, bounded cones and prisms shall satisfy the following properties.

Proposition 7. Given any two feasible state points $\left(l^{-}, v^{-}, t^{-}\right),\left(l^{+}, v^{+}, t^{+}\right)$and two quasi-trajectories

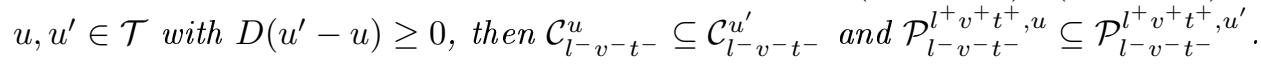

Then we will prove less intuitive properties for bounded cones and prisms.

Proposition 8. Given a feasible state point $\left(l, v, t^{-}\right)$and a trajectory $p^{\prime} \in \mathcal{T}$, then trajectory $p^{f}\left(\left(l, v, t^{-}\right), p^{\prime}\right)$ obtained from FSP is not empty $\Leftrightarrow \mathcal{C}_{l v t^{-}}^{p^{\prime}} \neq \emptyset \Leftrightarrow D\left(p^{\prime}-\underline{p}_{l v t^{-}}\right) \geq 0$.

Proof. It is apparent that $p^{\mathrm{f}}:=p^{\mathrm{f}}\left(\left(l, v, t^{-}\right), p^{\prime}\right) \neq \emptyset$ leads to $\mathcal{C}_{l v t^{-}}^{p^{\prime}} \neq \emptyset$ because $p^{\mathrm{f}} \in \mathcal{C}_{l v t^{-}}^{p^{\prime}}$. Further if we can find a feasible trajectory in $p \in \mathcal{C}_{l v t^{-}}^{p^{\prime}}$, we know that $D\left(p^{\prime}-p\right) \geq 0$ and $D\left(p-\underline{p}_{l v t^{-}}\right) \geq 0$, which yields $D\left(p^{\prime}-\underline{p}_{l v t^{-}}\right) \geq 0$ since $D$ is transitive. Now we only need to prove that $D\left(p^{\prime}-\underline{p}_{l v t^{-}}\right) \geq 0$ leads to $p^{\mathrm{f}}\left(\left(l, v, t^{-}\right), p^{\prime}\right) \neq \emptyset$. Now we are given $p^{\prime}(t) \geq \underline{p}_{l v t^{-}}(t), \forall t \in\left[t^{-}, \infty\right)$. If $p^{\prime}(t) \geq \bar{p}_{l v t^{-}}(t), \forall t \in\left[t^{-}, \infty\right)$, then Proposition 5 indicates $p^{\mathrm{f}}\left(\left(l, v, t^{-}\right), p^{\prime}\right)=p^{\mathrm{f}}\left(\left(l, v, t^{-}\right), \emptyset\right)$ that overlaps $\bar{p}_{l v t^{-}}\left(t^{-}: \emptyset\right)$. Therefore $p^{\mathrm{f}}\left(\left(l, v, t^{-}\right), p^{\prime}\right)$ should be always non-empty. Otherwise, it should be that $p^{\prime}(t) \geq \bar{p}_{l v t^{-}}(t), \forall t \in\left[t^{-}, t^{*}\right), p^{\prime}\left(t^{*}\right)=\bar{p}_{l v t^{-}}\left(t^{*}\right)$ and $p^{\prime}(t)<\bar{p}_{l v t^{-}}(t), \forall t \in\left(t^{*}, \infty\right)$ for some $t^{*} \in\left[t^{-}, \infty\right)$. We first define a continuous function of time $\hat{t} \in\left[t^{-}, \infty\right)$ as follows. We construct a trajectory denoted by $\underline{p}_{\hat{t}}$ composed by maximally accelerating section $\bar{p}_{l v t^{-}}\left(t^{-}: \hat{t}\right)$

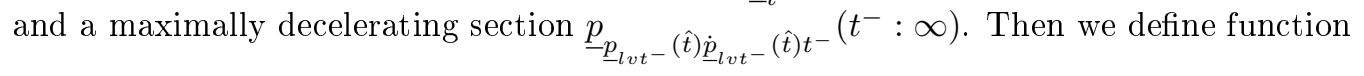

$$
d(\hat{t}):=D\left(p^{\prime}-\underline{p}_{\hat{t}}\right)=\min _{t \in\left[t^{-}, \infty\right)} p^{\prime}(t)-\underline{p}_{\hat{t}}(t) .
$$


Note that as $\hat{t}$ increases continuously, $\underline{p}_{\hat{t}}(t)$ increases continuously at every $t \in\left[t^{-}, \infty\right)$. Then we can see that function $d(\hat{t})$ shall continuously decrease with $\hat{t}$. Note that $\underline{p}_{\hat{t}}$ is identical to $\underline{p}_{l v t^{-}}$when $\hat{t}=0$. Then since $p^{\prime}(t) \geq \underline{p}_{l v t^{-}}(t), \forall t \in\left[t^{-}, \infty\right)$, we obtain $d(0) \geq 0$. Further, as $\hat{t}$ increases to $t^{*}$, then $\underline{p}_{\hat{t}}(t)$ and $p^{\prime}(t)$ shall intersection at $t^{*}$, which indicates that $d\left(t^{*}\right) \leq 0$. Due to Bolzano's Theorem (Apostol, 1969), we can always find a $\hat{t}^{\mathrm{m}} \in\left[t^{-}, t^{*}\right]$ such that $d\left(\hat{t}^{\mathrm{m}}\right)=0$, which indicates that $p^{\prime}$ and $\underline{p}_{\hat{t} \mathrm{~m}}$ get tangent at a time $\bar{t}^{\mathrm{m}} \in\left[\hat{t}^{\mathrm{m}}, \infty\right)$. Then a trajectory $p^{\mathrm{f}}$ can be obtained by concatenating $\bar{p}_{l v t^{-}}\left(t^{-}: \hat{t}^{\mathrm{m}}\right),\left(\bar{p}_{l v t^{-}}\left(\hat{t}^{\mathrm{m}}\right), \dot{\bar{p}}_{l v t^{-}}\left(\hat{t}^{\mathrm{m}}\right), \underline{a}, \hat{t}^{\mathrm{m}}, t^{\mathrm{m}}\right)$, $p^{\prime}\left(t^{\mathrm{m}}: \infty\right)$. Note that $p^{\mathrm{f}}$ is exactly $p^{\mathrm{f}}\left(\left(l, v, t^{-}\right), p^{\prime}\right)$ and thus $p^{\mathrm{f}}\left(\left(l, v, t^{-}\right), p^{\prime}\right)$ is not empty. This completes the proof.

We can further extend this result to a quasi-trajectory as an upper bound.

Corollary 2. Given a feasible state point $\left(l, v, t^{-}\right)$and a quasi-trajectory $u \in \mathcal{U}$, then trajectory $\left.p_{(}\left(l, v, t^{-}\right), u\right)$ obtained from the EFSP is not empty $\Leftrightarrow \mathcal{C}_{l v t^{-}}^{u} \neq \emptyset \Leftrightarrow D\left(u-\underline{p}_{l v t^{-}}\right) \geq 0$.

Proposition 9. Given a feasible state point $\left(l, v, t^{-}\right)$, a quasi-trajectory $u \in \mathcal{U}$ and scalars $\delta, \delta^{\prime} \geq 0$, if $\mathcal{C}_{l v t}^{u} \neq \emptyset$, then $\mathcal{C}_{(l-\delta) v\left(t^{-}+\delta^{\prime}\right)}^{u} \neq \emptyset$ and we obtain $D\left(p^{f}\left(\left(l, v, t^{-}\right), u\right)-p\right) \geq 0, \forall p \in \mathcal{C}_{(l-\delta) v\left(t^{-}+\delta^{\prime}\right)}^{u}$.

Proof. We first prove if $\mathcal{C}_{l v t}^{u} \neq \emptyset$, then $\mathcal{C}_{(l-\delta) v\left(t^{-}+\delta^{\prime}\right)}^{u} \neq \emptyset$. Due to Corollary 2, we know $D\left(u-\underline{p}_{l v t^{-}}\right) \geq 0$. Since apparently $D\left(\underline{p}_{l v t^{-}}-\underline{p}_{(l-\delta) v\left(t^{-}+\delta^{\prime}\right)}\right) \geq 0$, we obtain $D\left(u-\underline{p}_{(l-\delta) v\left(t^{-}+\delta^{\prime}\right)}\right) \geq 0$ due to the transitive property of operator $D$. This yields $\mathcal{C}_{(l-\delta) v\left(t^{-}+\delta^{\prime}\right)}^{u} \neq \emptyset$ based on Corollary 2.

Then we prove the remaining part of this proposition. In case that $D\left(u-\bar{p}_{l v t^{-}}\right) \geq 0, p^{\mathrm{f}}:=p^{\mathrm{f}}\left(\left(l, v, t^{-}\right), u\right)$ shall be identical to $\bar{p}_{l v t^{-}}$and thus $D\left(p^{\mathrm{f}}-p\right) \geq 0$ obviously holds $\forall p \in \mathcal{C}_{l v t^{-}}^{u}=\mathcal{C}_{l v t^{-}}$. Otherwise, we know that $p^{\mathrm{f}}=\left[\bar{p}_{l v t^{-}}\left(t^{-}: t^{\mathrm{m}}\right), \mathrm{s}^{\mathrm{m}}:=\left(\bar{p}_{l v t^{-}}\left(\hat{t}^{\mathrm{m}}\right), \dot{\bar{p}}_{l v t^{-}}\left(\hat{t}^{\mathrm{m}}\right), \underline{a}, \hat{t}^{\mathrm{m}}, \bar{t}^{\mathrm{m}}\right), p^{\prime}\left(t^{+}: \infty\right)\right]$ for some $t^{\mathrm{m}}$ and $t^{+}$ satisfying $t^{-} \leq t^{\mathrm{m}} \leq t^{+} \leq \infty$, where $p^{\prime}$ is the lower-bound trajectory merged by all elements in $u$ with EFSO. If $\exists p \in \mathcal{C}_{l v t^{-}}^{u}$ such that $D\left(p^{\mathrm{f}}-p\right)<0$, then there much exist a $\tilde{t} \in\left(t^{\mathrm{m}}, t^{+}\right)$such that $p(\tilde{t})$ is strictly above $\mathbf{s}^{\mathrm{m}}$. Since $D\left(\bar{p}_{l v t^{-}}-p\right) \geq 0$ and $D\left(p-\underline{p}_{p(\tilde{t}) \dot{p}(\tilde{t}) \tilde{t}}\right) \geq 0$, thus we have $D\left(\bar{p}_{l v t^{-}}-\underline{p} p(\tilde{t}) \dot{p}(\tilde{t}) \tilde{t}\right) \geq 0$, which indicates $\underline{p}_{p(\tilde{t}) \dot{p}(\tilde{t}) \tilde{t}}$ and $\mathbf{s}^{\mathrm{m}}$ have to intersect at a time $t^{\prime} \in\left[\hat{t}^{\mathrm{m}}, \tilde{t}\right)$. However, sine $D(u-p) \geq 0$, thus $\underline{p}_{p(\tilde{t}) \dot{p}(\tilde{t}) \tilde{t}}$ needs to intersect with $\mathbf{s}^{\mathrm{m}}$ at another time $t^{\prime \prime} \in\left(\tilde{t}, t^{\mathrm{m}}\right]$. This is contradictory to the fact that $\mathbf{s}^{\mathrm{m}}$ has already decelerated at the extreme deceleration rate $\underline{a}$. This contradiction proves that $D\left(p^{\mathrm{f}}-p\right) \geq 0, \forall p \in \mathcal{C}_{l v t^{-}}^{p^{\prime}}$. Further, given $\delta, \delta^{\prime} \geq 0$, for any $p \in \mathcal{C}_{(l-\delta) v\left(t^{-}+\delta^{\prime}\right)}^{u}$, we can find a $p^{\prime} \in \mathcal{C}_{l v t^{-}}^{p^{\prime}}$ satisfying $D\left(p^{\prime}-p\right) \geq 0$ with a similar argument. This proves that $D\left(p^{\mathrm{f}}-p^{\prime}\right) \geq 0, \forall p^{\prime} \in \mathcal{C}_{(l-\delta) v\left(t^{-}+\delta^{\prime}\right)}^{u}, \delta, \delta^{\prime} \geq 0$.

Symmetrically, we can obtain similar properties for BSP as well.

Corollary 3. Given a feasible state point $\left(l, v, t^{+}\right)$and a quasi-trajectory $u \in \mathcal{U}$, trajectory $p^{e b}\left(\left(l, v, t^{+}\right), u\right)$ obtained from BSP is not empty $\Leftrightarrow \mathcal{C}_{l v t^{+}}^{u} \neq \emptyset \Leftrightarrow D\left(u-\underline{p}_{p v t^{+}}\right) \geq 0$. If $\mathcal{C}_{l v t^{+}}^{u} \neq \emptyset$, we obtain $\mathcal{C}_{(l-\delta) v\left(t^{+}+\delta^{\prime}\right)}^{u} \neq \emptyset$ and $D\left(p^{e b}-p\right) \geq 0, \forall p \in \mathcal{C}_{(l-\delta) v\left(t^{+}+\delta^{\prime}\right)}^{u}$.

Combining Corollaries 2 and 3 leads to the following property with respect to a quadratic prism.

Corollary 4. Given two feasible state point $\left(l^{-}, v^{-}, t^{-}\right),\left(l^{+}, v^{+}, t^{+}\right)$satisfying $D\left(\bar{p}_{l^{-} v^{-} t^{-}}-\underline{p}_{l^{+} v^{+} t^{+}}\right) \geq 0$ and $D\left(\bar{p}_{l^{+} v^{+} t^{+}}-\underline{p}_{l^{-} v^{-} t^{-}}\right) \geq 0$, and a quasi-trajectory $u \in \mathcal{U}$, then $p^{f}\left(\left(l^{-}, v^{-}, t^{-}\right), u\right) \neq \emptyset$ and $p^{e b}\left(\left(l^{+}, v^{+}, t^{+}\right), u\right) \neq$ $\emptyset \Leftrightarrow \mathcal{P}_{l^{-} v^{-} t^{-}}^{l^{+}, u} \neq \emptyset \Leftrightarrow D\left(u-\underline{p}_{l^{-} v^{-} t^{-}}\right) \geq 0$ and $D\left(u-\underline{p}_{l^{+} v^{+} t^{+}}\right) \geq 0$. Whenever $\mathcal{P}_{l^{-} v^{-} t^{+}}^{l^{+} t^{+}, u} \neq \emptyset$, we obtain $D\left(p^{e b}-p\right) \geq 0, \forall p \in \mathcal{P}_{\left(l^{-}-\epsilon\right) v^{-}\left(t^{-}+\epsilon^{\prime}\right)}^{\left(l^{+}-\delta\right) v^{+}\left(t^{+}+\delta^{\prime}\right),}, \delta, \delta^{\prime}, \epsilon, \epsilon^{\prime} \geq 0$. 


\subsection{Feasibility Properties}

Although the proposed heuristic algorithms are essentially heuristics that may not explore the entire feasible region of the original problem, it can be used as a touchstone for the feasibility of the original problems under certain mild conditions. This section will discuss the relationships between the feasibility of the SH solutions and that of the original problems. Note that with different acceleration values, the $\mathrm{SH}$ algorithm yields different solutions. For uniformity, this section only investigates the representative SH algorithm with $\bar{a}^{\mathrm{f}}=\bar{a}^{\mathrm{b}}=\bar{a}$ and $\underline{a}^{\mathrm{f}}=\underline{a}^{\mathrm{b}}=\underline{a}$.

The following analysis investigates LVP, i.e., the feasibility of $\mathcal{P}^{\mathrm{LVP}}$ and that of $P^{\mathrm{PSH}}(\underline{a}, \bar{a})$.

Definition 17. Entry boundary condition $\left[v_{n}^{-}, t_{n}^{-}\right]_{n \in \mathcal{N}}$ is proper if $v_{n}^{-} \in[0, \bar{v}], \forall n \in \mathcal{N}$ and

$$
D\left(\bar{p}_{(m s-n s) v_{m}^{-}\left(t_{m}^{-}+n \tau-m \tau\right)}-\underline{p}_{0 v_{n}^{-} t_{n}^{-}}\right) \geq 0, \forall m<n \in \mathcal{N}
$$

Proposition 10. $\mathcal{P} \neq \emptyset \Rightarrow\left[v_{n}^{-}, t_{n}^{-}\right]_{n \in \mathcal{N}}$ is proper.

Proof. When $\mathcal{P} \neq \emptyset$, let $P=\left[p_{n}\right]_{n \in \mathcal{N}}$ denote a generic feasible trajectory vector in $\mathcal{P}$. For any given $m<n \in \mathcal{N}$, based on safety constraint (4), we obtain $D\left(p_{m}^{s}-p_{m+1}\right) \geq 0, D\left(p_{m+1}^{s}-p_{m+2}\right) \geq 0, \cdots$, $D\left(p_{n-1}^{s}-p_{n}\right) \geq 0$. This can be translated as $D\left(p_{m}^{s^{n-m}}-p_{m+1}^{s^{n-m-1}}\right) \geq 0, D\left(p_{m+1}^{s^{n-m-1}}-p_{m+2}^{s^{n-m-2}}\right) \geq 0, \cdots$, $D\left(p_{n-1}^{s}-p_{n}\right) \geq 0$, which indicates that $D\left(p_{m}^{s^{n-m}}-p_{n}\right) \geq 0$ due to the transitive property of function $D(\cdot)$. Further, since $D\left(\bar{p}_{(m s-n s) v_{m}^{-}\left[t_{m}^{-}+(n-m) \tau\right]}-p_{m}^{s^{n-m}}\right) \geq 0$ and $D\left(p_{n}-\underline{p}_{0 v_{n}^{-}} t_{n}^{-}\right) \geq 0$, we obtain $D\left(\bar{p}_{(m s-n s) v_{m}^{-}\left[t_{m}^{-}+(n-m) \tau\right]}-\underline{p}_{0 v_{n}^{-} t_{n}^{-}}\right) \geq 0, \forall m<n \in \mathcal{N}$. This completes the proof.

Theorem 1. $\mathcal{P}^{L V P} \neq \emptyset \Leftrightarrow P^{\mathrm{PSH}}(\underline{a}, \bar{a}) \neq \emptyset \Leftrightarrow\left[v_{n}^{-}, t_{n}^{-}\right]_{n \in \mathcal{N}}$ is proper.

Proof. When $\left[v_{n}^{-}, t_{n}^{-}\right]_{n \in \mathcal{N}}$ is proper, based on Corollary 3, we know that every EFSP step will generate a feasible trajectory. Thus $P^{\mathrm{PSH}}(\underline{a}, \bar{a})$ is feasible. Since $P^{\mathrm{PSH}}(\underline{a}, \bar{a}) \in \mathcal{P}^{\mathrm{LVP}}$, we see that $\mathcal{P}^{\mathrm{LVP}}$ is feasible, too. Further, Proposition 10 indicates that when $\mathcal{P}^{\mathrm{LVP}}$ is feasible, which means $\mathcal{P}$ too is feasible, $\left[v_{n}^{-}, t_{n}^{-}\right]_{n \in \mathcal{N}}$ is proper. This completes the proof.

Now we add back the signal control and investigate the feasibility of $\mathcal{P}$ under milder conditions with the SH solution. We consider a special subset of $\mathcal{P}$ where every trajectory has the maximum speed of $\bar{v}$ at the exit location $L$, i.e,

$$
\hat{\mathcal{P}}:=\left\{\left[p_{n}\right]_{n \in \mathcal{N}} \in \mathcal{P} \mid \dot{p}_{n}\left(p_{n}^{-1}(L)\right)=\bar{v}, \forall n \in \mathcal{N}\right\} .
$$

This subset is not too restrictive, because in order to assure a high traffic throughput rate, the exist speed of each vehicle should be high. The following analysis investigates the relationship between $\hat{\mathcal{P}}$ and $P^{\mathrm{SH}}(\underline{a}, \bar{a}, \underline{a}, \bar{a})$.

Proposition 11. When $L \geq \bar{v}^{2} /(2 \bar{a})$, if $P^{S H}(\underline{a}, \bar{a}, \underline{a}, \bar{a})=\left[p_{n}\right]_{n \in \mathcal{N}}$ is feasible, then $\dot{p}_{n}(t)=\bar{v}, \forall t \geq$ $p_{n}^{-1}(L), n \in \mathcal{N}$.

Proof. We use induction to prove this proposition. The induction assumption is , $p_{n}(t)=\bar{v}, \forall t \geq p_{n}^{-1}(L)$. If the BSP is not needed, Proposition 5 indicates that $p_{1}=p_{1}^{\mathrm{f}}=\bar{p}_{0 v_{1}^{-} t_{1}^{-}}\left(t_{1}^{-}: \infty\right)$ and thus since $L \geq \bar{v}^{2} /(2 \bar{a})$, we obtain $\dot{p}_{1}(t)=\dot{\bar{p}}_{0 v_{1}^{-} t_{1}^{-}}(t)=\bar{v}, \forall t \geq p_{1}^{-1}(L)=\bar{p}_{0 v_{1}^{-} t_{1}^{-}}^{-1}(L)$. Otherwise, if BSP is activated, it shoots backwards from the state point $\left(L, \bar{p}_{0 v_{1}^{-} t_{1}^{-}}\left(\bar{p}_{0 v_{1}^{-} t_{1}^{-}}^{-1}(L)\right)=\bar{v}, G\left(\bar{p}_{0 v_{1}^{-} t_{1}^{-}}^{-1}(L)\right)\right)$, and thus Proposition 6 indicates that

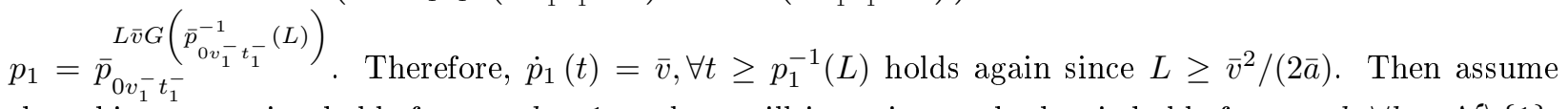
that this assumption holds for $n=k-1$, and we will investigate whether it holds for $n=k, \forall k \in \mathcal{N} \backslash\{1\}$. 
If the primary FSP is not blocked by $p_{k-1}^{\mathrm{S}}$, apparently $p_{k}=\bar{p}_{0 v_{k}^{-}} t_{k}^{-}$if the BSP is not needed, or $p_{k}=$

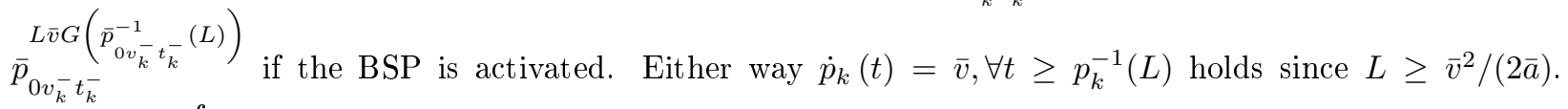
Otherwise, $p_{k}^{\mathrm{f}}$ merges into $p_{k-1}^{\mathrm{S}}$ before reaching $L$, and thus based on the induction assumption we obtain $\dot{p}_{k}^{\mathrm{f}}\left(p_{k}^{\mathrm{f}-1}(L)\right)=\dot{p}_{k-1}^{\mathrm{S}}\left(p_{k-1}^{\mathrm{S}-1}(L)\right)=\dot{p}_{k-1}\left(p_{k-1}^{-1}(L+s)\right)=\bar{v}$. Note that again the BSP could only shift segments in parallel and thus $\dot{p}_{k}\left(p_{k}^{-1}(L)\right)=\dot{p}_{k}^{\mathrm{f}}\left(p_{k}^{\mathrm{f}-1}(L)\right)=\bar{v}$. The induction assumption also indicates that the segments of $p_{k-1}$ used in the auxiliary FSP for $p_{k}$ is at constant speed $\bar{v}$. This means that the auxiliary FSP for $p_{k}$ will not be blocked by $p_{k-1}$, and thus we obtain $\dot{p}_{k}(t)=\bar{v}, \forall t \geq p_{k}^{-1}(L)$. This completes the proof.

Theorem 2. When $L \geq \bar{v}^{2} /(2 \bar{a}), \hat{\mathcal{P}} \neq \emptyset \Leftrightarrow P^{S H}(\underline{a}, \bar{a}, \underline{a}, \bar{a}) \neq \emptyset$.

Proof. The proof of the sufficiency is simple. Again, we can write $P^{\mathrm{SH}}(\underline{a}, \bar{a}, \underline{a}, \bar{a})$ as $\left[p_{n}\right]_{n \in \mathcal{N}}$. When $P^{\mathrm{SH}}(\underline{a}, \bar{a}, \underline{a}, \bar{a}) \neq \emptyset$, Proposition 11 indicates that $P \in \hat{\mathcal{P}}$ and thus $\hat{\mathcal{P}} \neq \emptyset$.

Then we only need to prove the necessity. When there exists $P^{\prime}=\left[p_{n}^{\prime}\right]_{n \in \mathcal{N}} \in \hat{\mathcal{P}}$ that is not empty, we will show that $\left[p_{n}\right]_{n \in \mathcal{N}}$ too is not empty with the following induction. For the notation convenience, we denote $t_{n}^{\prime+}=\left(p_{n}^{\prime}\right)^{-1}(L)$ and $t_{n}^{+}:=p_{n}^{-1}(L)$. The induction assumption is that $p_{n}$ exists and $D\left(p_{n}-p_{n}^{\prime}\right) \geq 0$. When $n=1$, if BSP is not activated in constructing $p_{1}$, then $p_{1}=p^{\mathrm{f}}\left(\left(0, v_{1}^{-}, t_{1}^{-}\right), \emptyset\right)=\bar{p}_{0 v_{1}^{-} t_{1}^{-}}\left(t^{-}: \infty\right)$ based on Proposition 5. Therefore, $p_{1}$ exists and $D\left(p_{1}-p_{1}^{\prime}\right) \geq 0$. Otherwise, denote $\bar{t}_{1}^{+}=\bar{p}_{0 v_{1}^{-} t_{1}^{-}}^{-1}(L)$ and $t_{1}^{+}:=G\left(\bar{t}_{1}^{+}\right)$. Note that since $L \geq \bar{v}^{2} /(2 \bar{a}), \dot{\bar{p}}_{0 v_{1}^{-} t_{1}^{-}}\left(\bar{p}_{0 v_{1}^{-} t_{1}^{-}}(L)\right)=\bar{v}$. Apparently, $D\left(\bar{p}_{0 v_{1}^{-} t_{1}^{-}}-\underline{p}_{L \bar{v} \bar{t}_{1}^{+}}\right) \geq 0$ and $D\left(\underline{p}_{L \bar{v} \bar{t}_{1}^{+}}-\underline{p}_{L \bar{v} t_{1}^{+}}\right) \geq 0$, which indicates $D\left(\bar{p}_{0 v_{1}^{-} t_{1}^{-}}-\underline{p}_{L \bar{v} t_{1}^{+}}\right) \geq 0$. Further since $L \geq \bar{v}^{2} /(2 \bar{a})$, we know $D\left(\bar{p}_{L \bar{v} t_{1}^{+}}-\underline{p}_{0 v_{1}^{-} t_{1}^{-}}\right) \geq 0$. Then we obtain $p_{1}=\bar{p}_{0 v_{1}^{-} t_{1}^{-}}$based on Proposition 6. Further, apparently $t_{1}^{+} \geq \bar{t}_{1}^{+}$, and then $t_{1}^{\prime+}=G\left(t_{1}^{\prime}\right) \geq G\left(\bar{t}_{1}^{+}\right)=t_{1}^{+}$since function $G(\cdot)$ is increasing. Then Proposition 4 indicates that $D\left(p_{1}-p_{1}^{\prime}\right) \geq 0$.

Then assume that the induction assumption holds for $n=k-1, \forall k \in \mathcal{N} \backslash\{1\}$. Then for $n=k$, Since

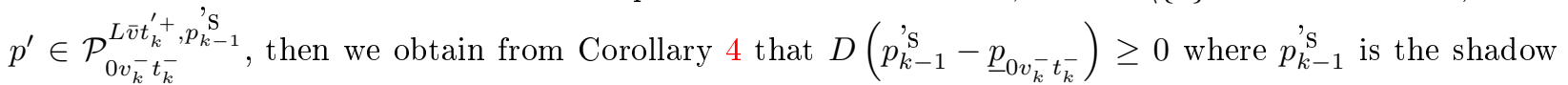
trajectory of $p_{k-1}^{\prime}$. And the induction assumption tells that $D\left(p_{k-1}^{\mathrm{s}}-p_{k-1}^{\mathrm{s}}\right) \geq 0$ where $p_{k-1}^{\mathrm{S}}$ is the shadow trajectory of $p_{k-1}$, which further indicates that $D\left(p_{k-1}^{\mathrm{S}}-\underline{p}_{0 v_{k}^{-}} t_{k}^{-}\right) \geq 0$ based on the transitive property of $D$. Then Proposition 8 indicate that $p^{\mathrm{f}}\left(\left(0, v_{k}^{-}, t_{k}^{-}\right), p_{k-1}^{\mathrm{S}}\right) \neq \emptyset$. For the simplicity of presentation, we denote $p^{\mathrm{f}}\left(\left(0, v_{k}^{-}, t_{k}^{-}\right), p_{k-1}^{\mathrm{s}}\right)$ by $p_{k}^{\mathrm{f}}$. If BSP is not used in constructing $p_{k}$, then $p_{k}=p_{k}^{\mathrm{f}}$ exists and Proposition 9 indicates $D\left(p_{k}-p_{k}^{\prime}\right) \geq 0$. Otherwise, let $\bar{t}_{k}^{+}=p_{k}^{\mathrm{f}-1}(L)$ and $t_{k}^{+}=G\left(\bar{t}_{k}^{+}\right)$. Then if $p_{k}$ exists, then $p_{k}^{-1}(L)=t_{k}^{+}$and $\dot{p}_{k}\left(t_{k}^{+}\right)=\bar{v}$ since $L \geq \bar{v}^{2} /(2 \bar{a})$. Again, it is easy to see that $D\left(\bar{p}_{0 v_{k}^{-} t_{k}^{-}}-\underline{p}_{L \bar{v} t_{k}^{+}}\right) \geq 0$ and $D\left(\bar{p}_{L \bar{v} t_{k}^{+}}-\underline{p}_{0 v_{k}^{-}} t_{k}^{-}\right) \geq 0$, and therefore Corollary 4 indicates that $p_{k}$ exists. Also, apparently $t_{k}^{\prime+} \geq \bar{t}_{k}^{+}$, and then $t_{k}^{\prime+}=G\left(t_{k}^{+}\right) \geq G\left(\bar{t}_{k}^{+}\right)=t_{k}^{+}$since function $G(\cdot)$ is increasing. Note that $p_{k}=p^{\mathrm{eb}}\left(\left(L, \bar{v}, t_{k}^{+}\right), p_{k}^{\mathrm{f}}\right)$ from BSP with $\bar{a}^{\mathrm{f}}=\bar{a}^{\mathrm{b}}=\bar{a}$ and $\underline{a}^{\mathrm{f}}=\underline{a}^{\mathrm{b}}=\underline{a}$. Then Corollary 4 further indicates that $D\left(p_{k}-p_{k}^{\prime}\right) \geq 0$. This completes the proof.

Further, we will show that when $L$ is sufficiently long, the feasibility of $P^{\mathrm{SH}}(\underline{a}, \bar{a}, \underline{a}, \bar{a})$ is equivalent to the feasibility of $\mathcal{P}$.

Theorem 3. When $L \geq \frac{\bar{v}^{2}}{2 \bar{a}}+\frac{\bar{v}^{2}}{-2 \underline{a} b}+\frac{\bar{v}^{2}}{-2 \underline{a} b}+s(N-1), P^{S H}(\underline{a}, \bar{a}, \underline{a}, \bar{a}) \neq \emptyset \Leftrightarrow \mathcal{P} \neq \emptyset \Leftrightarrow\left[v_{n}^{-}, t_{n}^{-}\right]_{n \in \mathcal{N}}$ is proper. 
Proof. When $P^{\mathrm{SH}}(\underline{a}, \bar{a}, \underline{a}, \bar{a}) \neq \emptyset$, it is trivial to see that $\mathcal{P} \neq \emptyset$ since $P^{\mathrm{SH}}(\underline{a}, \bar{a}, \underline{a}, \bar{a}) \in \mathcal{P}$. Proposition 10 indicates that $\mathcal{P} \neq \emptyset$ leads to that $\left[v_{n}^{-}, t_{n}^{-}\right]_{n \in \mathcal{N}}$ is proper. Then we only need to prove that when $\left[v_{n}^{-}, t_{n}^{-}\right]_{n \in \mathcal{N}}$ is proper, $P^{\mathrm{SH}}(\underline{a}, \bar{a}, \underline{a}, \bar{a}) \neq \emptyset$. We denote the trajectories in $P^{\mathrm{SH}}(\underline{a}, \bar{a}, \underline{a}, \bar{a})$ with $\left[p_{n}\right]_{n \in \mathcal{N}}$. Note that for each trajectory $p_{n}$, if BSP in SH3 is activated, then it shall be always successfully completed within highway segment $\left[\hat{L}:=L-\bar{v}^{2} /\left(-2 \bar{a}^{\mathrm{b}}\right)-\bar{v}^{2} /\left(-2 \bar{a}^{\mathrm{b}}\right), L\right]$. Therefore, BSP neither causes infeasibility nor affects the shape of $p_{\mathrm{n}}$ within $\left[0, \hat{L}_{\mathrm{n}}:=L-\bar{v}^{2} /\left(-2 \bar{a}^{\mathrm{b}}\right)-\bar{v}^{2} /\left(-2 \bar{a}^{\mathrm{b}}\right)-s(n-1)\right]$. Even if we consider the backward wave propagation caused by the previous trajectories, this indicates $p_{n}\left(t_{n}^{-}: \dot{p}_{n}^{-1}\left(\hat{L}_{\mathrm{n}}\right)\right)=$ $p_{n}^{\mathrm{f}}\left(t_{n}^{-}: \dot{p}_{n}^{-1}\left(\hat{L}_{\mathrm{n}}\right)\right)$, and if the feasibility check of $p_{n}$ fails in $\mathrm{SH}$, it must happen in this section. Note that Theorem 1 shows that if $\left[v_{n}^{-}, t_{n}^{-}\right]_{n \in \mathcal{N}}$ is proper, $P^{\mathrm{PSH}}(\underline{a}, \bar{a})$ is not empty. We denote $P^{\mathrm{PSH}}(\underline{a}, \bar{a})$ with $\left[\hat{p}_{n}\right]_{n \in \mathcal{N}}$. Then to prove this theorem, we only need to show that $p_{n}\left(t_{n}^{-}: p_{n}^{-1}\left(\hat{L}_{n}\right)\right)=\hat{p}_{n}\left(t_{n}^{-}: p_{n}^{-1}\left(\hat{L}_{n}\right)\right), \forall n \in$ $\mathcal{N}$ from the following induction.

The induction assumption is $p_{n}\left(t_{n}^{-}: p_{n}^{-1}\left(\hat{L}_{n}\right)\right)=\hat{p}_{n}\left(t_{n}^{-}: p_{n}^{-1}\left(\hat{L}_{n}\right)\right)$ and $\dot{p}_{n}(t)=\bar{v}, \forall t \in\left[p_{n}^{-1}\left(\hat{L}_{n}\right), p_{n}^{-1}(\hat{L})\right]$. When $n=1$, the trajectory generated from FSP satisfies $p_{1}^{\mathrm{f}}:=p_{1}^{\mathrm{f}}\left(\left(0, v_{1}^{-}, t_{1}^{-}\right), \emptyset\right)=\hat{p}_{n}$. Since BSP will not affect the shape of $p_{1}$ below $\hat{L}_{1}$, thus $p_{1}\left(t_{1}^{-}: p_{1}^{-1}\left(\hat{L}_{1}\right)\right)=\hat{p}_{1}\left(t_{1}^{-}: p_{1}^{-1}\left(\hat{L}_{1}\right)\right)$. Since $p_{1}^{\mathrm{f}}$ shall finish accelerating to $\bar{v}$ before reaching location $\hat{L}$ based on the value of $\hat{L}, \dot{p}_{1}(t)=\dot{\hat{p}}_{1}(t)=\bar{v}, \forall t \in\left[p_{n}^{-1}\left(\hat{L}_{n}\right), p_{n}^{-1}(\hat{L})\right]$. Then we assume that the induction assumption holds for $n=k-1, \forall k \in \mathcal{N} \backslash\{1\}$. When $n=k$, the forward shooting trajectory is $p_{k}^{\mathrm{f}}:=p^{\mathrm{f}}\left(\left(0, v_{k}^{-}, t_{k}^{-}\right), p_{k-1}^{\mathrm{S}}\right)$. If $D\left(p_{k-1}^{\mathrm{S}}-\bar{p}_{0 v_{k}^{-}} t_{k}^{-}\right) \geq 0$, then apparently $p_{k}^{\mathrm{f}}=\hat{p}_{k}=p^{\mathrm{f}}\left(\left(0, v_{k}^{-}, t_{k}^{-}\right), \emptyset\right)$, and thus $p_{k}\left(t_{k}^{-}: p_{k}^{-1}\left(\hat{L}_{k}\right)\right)=p_{k}^{\mathrm{f}}\left(t_{k}^{-}: p_{k}^{-1}\left(\hat{L}_{k}\right)\right)=\hat{p}_{k}\left(t_{k}^{-}: p_{k}^{-1}\left(\hat{L}_{k}\right)\right)$ and $\dot{p}_{k}(t)=\bar{v}, \forall t \in\left[p_{k}^{-1}\left(\hat{L}_{k}\right), p_{k}^{-1}(\hat{L})\right]$. Otherwise, note that $p_{k}^{\mathrm{f}}=p^{\mathrm{f}}\left(\left(0, v_{k}^{-}, t_{k}^{-}\right), p_{k-1}^{s}\right)=p^{\mathrm{f}}\left(\left(0, v_{k}^{-}, t_{k}^{-}\right), \hat{p}_{k-1}^{s}\right)=\hat{p}_{k}$ and thus the existence of $\hat{p}_{k}$ indicates that $p_{k}^{\mathrm{f}}$ exists as well. The induction assumption of $\dot{p}_{k-1}(t)=\bar{v}, \forall t \in$ $\left[p_{k-1}^{-1}\left(\hat{L}_{k-1}\right), p_{k-1}^{-1}(\hat{L})\right]$ indicates that $\dot{p}_{k-1}^{\mathrm{S}}(t)=\bar{v}, \forall t \in\left[p_{k-1}^{\mathrm{S}-1}\left(\hat{L}_{k}=\hat{L}_{k-1}-s\right), p_{k-1}^{\mathrm{S}-1}(\hat{L}-s)\right]$, and therefore, $p_{k}^{\mathrm{f}}$ shall merge with $p_{k-1}^{\mathrm{S}}$ at a location before $\hat{L}$ because the merging speed has to be less than $\bar{v}$, and therefore $\dot{p}_{k}(t)=\dot{p}_{k}^{\mathrm{f}}(t)=\bar{v}, \forall t \in\left[p_{k}^{-1}\left(\hat{L}_{k}\right), p_{k}^{-1}(\hat{L})\right]$. Therefore, $p_{k}$ and $\hat{p}_{k}$ overlap over highway segment $\left[0, \hat{L}_{n}\right]$, or $p_{k}\left(t_{k}^{-}: p_{k}^{-1}\left(\hat{L}_{k}\right)\right)=\hat{p}_{k}\left(t_{k}^{-}: p_{k}^{-1}\left(\hat{L}_{k}\right)\right)$. This completes the proof.

\subsection{Relationship to Classic Traffic Flow Models}

Evolution of highway traffic has been traditionally investigated with various microscopic models (e.g., car following (Brackstone and McDonald, 1999), cellular automata (Nagel and Schreckenberg, 1992)) and macroscopic kinematic models (kinemetic models (Lighthill and Whitham, 1955; Richards, 1956) and cell transmission (Daganzo, 1994)). Daganzo (2006) proves the equivalence between the kinematic wave model with the triangular fundamental diagram (KWT) (Newell, 1993), Newell's lower-order model (Newell, 2002) and the linear cellular automata model (Nagel and Schreckenberg, 1992). We will just show the relevance of the the SHL solution to Newell's lower-order model (or Newell's model), and this relevance can be easily transferred to other models based on their equivalence. Given the first vehicle's trajectory $q_{1}=p_{1} \in \mathcal{T}$, Newell's model specifies a rule to construct vehicle $n$ 's trajectory, denoted by $q_{n}$, with vehicle $n^{\prime} s$ entry condition $\left(0, \cdot, t_{n}^{-}\right)$ and preceding trajectory $q_{n-1}$, as formulated below

$$
q_{n}(t)=\min \left\{\bar{v}\left(t-t_{n}^{-}\right), q_{n-1}(t-\tau)-s\right\}, \forall n \in \mathcal{N} \backslash\{1\}, t \in\left[t_{n}^{-}, \infty\right) .
$$

For notation convenience, we denote this equation with $q_{n}=q^{\mathrm{KWT}}\left(\left(0, \cdot, t_{n}^{-}\right), q_{n-1}\right)$. This section analyzes the relationship between the SHL solution with Newell's solution for the same LVP setting. We denote the SHL trajectory vector with $P^{\mathrm{LVP}}\left(\underline{a}^{\mathrm{f}}, \bar{a}^{\mathrm{f}}\right)=\left[p_{n}\right]_{n \in \mathcal{N}}$ and that from Newell's model by $Q=\left[q_{n}\right]_{n \in \mathcal{N}}$ . Without loss of generality, we only investigate the case when $\left[v_{n}^{-}, t_{n}^{-}\right]_{n \in \mathcal{N}}$ is proper or $P^{\mathrm{LVP}}\left(\underline{a}^{\mathrm{f}}, \bar{a}^{\mathrm{f}}\right)$ is 
feasible.

Daganzo (2006) showed that Newell's model has a contraction property; i.e., the result of Newell's model is insensitive to small input errors, as stated in the following proposition.

Proposition 12. Given two trajectories $q, q^{\prime} \in \overline{\mathcal{T}}$ satisfying $\max _{t \in(-\infty, \infty)}\left|q(t)-q^{\prime}(t)\right| \leq \epsilon$ for some $\epsilon>$ 0 , then $\max _{t \in(-\infty, \infty)}\left|q^{K W T}\left(\left(0, \cdot, t^{-}\right), q^{s}\right)-q^{K W T}\left(\left(\delta, \cdot, t^{-}\right), q^{\prime} s\right)\right| \leq \epsilon$ for any $|\delta|<\epsilon$ and $t^{-}$yielding $q^{\mathrm{KWT}}\left(\left(0, \cdot, t^{-}\right), q^{\mathrm{S}}\right) \neq \emptyset$ and $q^{\mathrm{KWT}}\left(\left(\delta, \cdot, t^{-}\right), q^{\prime \mathrm{S}}\right) \neq \emptyset$.

We now show that $P^{\mathrm{LVP}}\left(\underline{a}^{\mathrm{f}}, \bar{a}^{\mathrm{f}}\right)$ has the same contraction property.

Theorem 4. Given two feasible trajectories $p, p^{\prime} \in \mathcal{T}$ satisfying $\max _{t \in(-\infty, \infty)}\left|p(t)-p^{\prime}(t)\right| \leq \epsilon$, then

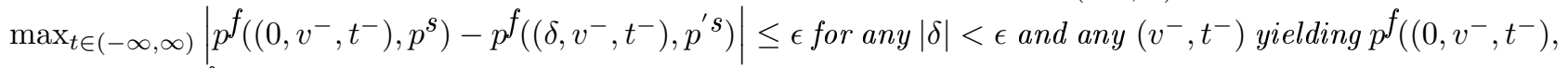
$\left.p^{s}\right) \neq \emptyset$ and $\left.p_{(}\left(\delta, v^{-}, t^{-}\right), p^{\prime} s\right) \neq \emptyset$.

Proof. Since $\max _{t \in(-\infty, \infty)}\left|p(t)-p^{\prime}(t)\right| \leq \epsilon$, we obtain $\max _{t \in(-\infty, \infty)}\left|p^{\mathrm{S}}(t)-p^{\prime} \mathrm{s}(t)\right| \leq \epsilon$. Further, define $p^{\mathrm{S}+\epsilon}(t):=p^{\mathrm{S}}(t)+\epsilon, p^{\mathrm{S}-\epsilon}(t):=p^{\mathrm{S}}(t)-\epsilon, p^{\mathrm{f}+\epsilon}:=p^{\mathrm{f}}\left(\left(\epsilon, v^{-}, t^{-}\right), p^{\mathrm{S}+\epsilon}\right)$ and $p^{\mathrm{f}_{-\epsilon}}:=p^{\mathrm{f}}\left(\left(-\epsilon, v^{-}, t^{-}\right), p^{\mathrm{S}-\epsilon}\right)$. Note that $p(t)=p^{+\epsilon}(t)-\epsilon=p^{-\epsilon}(t)+\epsilon, \forall t \in(-\infty, \infty)$. Since $D\left(p^{s+\epsilon}-p^{\mathbf{S}}\right)=\epsilon$ and $\max _{t \in(-\infty, \infty)}\left|p(t)-p^{\prime}(t)\right| \leq \epsilon$,

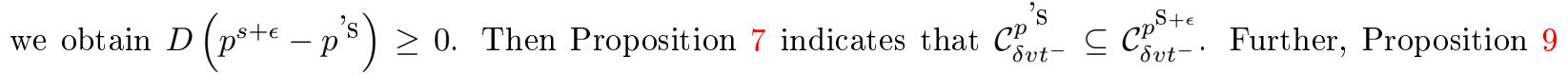
indicates $D\left(p^{\mathrm{f}+\epsilon}-\hat{p}\right) \geq 0, \forall \hat{p} \in \mathcal{C}_{\delta v t^{-}}^{p^{\mathrm{S}+\epsilon}}$. We denote $p^{\mathrm{f}}\left(\left(0, v^{-}, t^{-}\right), p^{\mathrm{S}}\right)$ and $p^{\mathrm{f}}\left(\left(\delta, v^{-}, t^{-}\right), p^{\prime \mathrm{s}}\right)$ as $p^{\mathrm{f}}$ and $p^{\prime \mathrm{f}}$ for short, respectively. Since $p^{\prime \mathrm{f}} \in \mathcal{C}_{\delta v t^{-}}^{p^{\prime} \mathrm{s}} \subseteq \mathcal{C}_{\delta v t^{-}}^{p^{\mathrm{S}+\epsilon}}$, we obtain $D\left(p^{\mathrm{f}+\epsilon}-p^{\prime \mathrm{f}}\right) \geq 0$. On the other hand, we obtain $D\left(p^{\text {'s }}-p^{s-\epsilon}\right) \geq 0$. Then Proposition 7 indicates $\mathcal{C}_{-\epsilon v t^{-}}^{p^{\mathrm{S}-\epsilon}} \subseteq \mathcal{C}_{-\epsilon v t^{-}}^{p^{\prime} \mathrm{s}}$. Further, Proposition 9 indicates $D\left(p^{\prime}-\hat{p}\right) \geq 0, \forall \hat{p} \subseteq \mathcal{C}_{-\epsilon v t^{-}}^{p^{\prime} \mathrm{s}}$. Since $p^{\mathrm{f}-\epsilon} \in \mathcal{C}_{-\epsilon v t^{-}}^{p^{\mathrm{S}-\epsilon}} \subseteq \mathcal{C}_{-\epsilon v t^{-}}^{p^{\prime} \mathrm{s}}$, we obtain $D\left(p^{\prime \mathrm{f}}-p^{\mathrm{f}-\epsilon}\right) \geq 0$. Combining $D\left(p^{\mathrm{f}+\epsilon}-p^{\prime \mathbf{f}}\right) \geq 0$ and $D\left(p^{\prime \mathbf{f}}-p^{\mathrm{f}-\epsilon}\right) \geq 0$ yields $\max _{t \in(-\infty, \infty)}\left|p^{\mathrm{f}}(t)-p^{\prime \mathbf{f}}(t)\right| \leq \epsilon$.

Theorem 4 impliesthat $P^{\mathrm{LVP}}\left(\underline{a}^{\mathrm{f}}, \bar{a}^{\mathrm{f}}\right)$ is not sensitive to small input errors as well. However, we shall note that the solution to the SH algorithm for a signalized highway segment may be sensitive to small errors because the exit time of a trajectory, if close to the start of a red phase, could be pushed back to the next green phase due to a small input perturbation. Nonetheless, this kind of "jump" only affects a limited number of trajectories that are close to a red phase, and most other trajectories will not be much affected.

The following analysis investigates the difference between $P^{\mathrm{LVP}}\left(\underline{a}^{\mathrm{f}}, \bar{a}^{\mathrm{f}}\right)$ and $Q$.

Proposition 13. $Q=\left\{q_{n}\right\}_{n \in \mathcal{N}}$ formulated in (11) can be solved as

$$
q_{n}(t)=\min \left\{p_{1}^{s^{n-1}}(t), \bar{v}\left(t-t_{n}^{-}\right)\right\}, \forall t \in\left[t_{n}^{-}, \infty\right), n \in \mathcal{N}
$$

where $p_{1}^{s^{k}}(t):=p_{1}(t-k \tau)-k s, \forall k$.

Proof. We prove this proposition with induction. Apparently, equation (12) holds for $n=1$ since $p_{1}^{\mathrm{S}^{0}}=p_{1} \in$ $\mathcal{T}$ and thus $\bar{v}\left(t-t_{1}^{-}\right) \geq p_{1}^{\mathrm{S}^{0}}(t), \forall t \in\left[t_{1}^{-}, \infty\right)$. Assume that equation (12) holds for $n=k-1$. When $n=k$, equation (11) indicates $q_{k}(t)=\min \left\{\bar{v}\left(t-t_{k}^{-}\right), q_{k-1}(t-\tau)-s\right\}$. Then plug the induction assumption into the above equation and we obtain $q_{k}(t)=\min \left\{\bar{v}\left(t-t_{k}^{-}\right), p_{1}^{\mathrm{S}^{k-2}}(t-\tau)-s, \bar{v}\left(t-t_{k-1}^{-}-\tau\right)-s\right\}=\min \left\{p_{1}^{\mathrm{S}^{k-1}}(t), \bar{v}(t-\right.$ $\left.\left.\max \left\{t_{k}^{-}, t_{k-1}^{-}+\tau+\bar{v} / s\right\}\right)\right\}$. Since $\left[v_{n}^{-}, t_{n}^{-}\right]_{n \in \mathcal{N}}$ is proper, we obtain $\max \left\{t_{k}^{-}, t_{k-1}^{-}+\tau+\bar{v} / s\right\}=t_{k}^{-} \mathrm{KWT}$. This completes the proof. 
Theorem 5. If $P^{L V P}\left(\underline{a}^{f}, \bar{a}^{f}\right) \neq \emptyset$ and $Q \neq \emptyset$, then $D\left(q_{n}-p_{n}\right)=0$ and

$$
D\left(p_{n}-q_{n}\right) \geq \min \left\{-0.5 \bar{v}^{2} / \bar{a}^{f}, 0.5 \bar{v}^{2} / \underline{a}^{f}\right\}, \forall n \in \mathcal{N} \backslash\{1\} .
$$

Proof. We compare parallel formulations from $P^{\mathrm{LVP}}\left(\underline{a}^{\mathrm{f}}, \bar{a}^{\mathrm{f}}\right)$ from PSHL in Section 3.2 with equation (12) for $Q$. We only need to prove this theorem for a generic $n \in \mathcal{N} \backslash\{1\}$. From PSHL, we see that $p_{n}$ is essentially obtained by smoothing quasi-trajectory $u_{n}:=u\left(\left\{\bar{p}_{m}^{\mathrm{S}^{n-m}}\right\}_{m=1, \cdots, n}\right)$ with tangent segments at constant decelerating rate $\underline{a}$. Define $\bar{q}_{n}(t):=\bar{v}\left(t-t_{n}^{-}\right), \forall t \in\left[t_{n}^{-},+\infty\right), \underline{q}_{n}:=p^{\mathrm{f}}\left(\left(0,0, \underline{t}_{n}\right), \emptyset\right)$. Note that $D\left(\underline{q}_{n}-\bar{q}_{n}\right)=-0.5 \bar{v}^{2} / \bar{a}^{\mathrm{f}}$, and $q_{n}(t)=\min \left\{\bar{q}_{n}(t), p_{1}^{\mathrm{S}^{n-1}}(t)\right\}$. Note that $D\left(q_{n}-u_{n}\right) \geq 0$ since $\bar{q}_{n} \geq \bar{p}_{n}$. Also, $D\left(u_{n}-p_{n}\right) \geq 0$ since the merging segments generated from EFSO-2 are all below $u_{n}$. This indicates $D\left(q_{n}-p_{n}\right) \geq 0$. Further since $q_{n}$ and $p_{n}$ always meet at the initial point $\left(0, t_{n}^{-}\right)$, we obtain $D\left(q_{n}-p_{n}\right)=0$. Then we will prove $D\left(p_{n}-q_{n}\right) \geq \min \left\{-0.5 \bar{v}^{2} / \bar{a}^{\mathrm{f}}, 0.5 \bar{v}^{2} / \underline{a}^{\mathrm{f}}\right\}$. We first examine $p_{n}^{\prime}:=p^{\mathrm{f}}\left(\left(0, v_{n}^{-}, t_{n}^{-}\right)\right.$, $\left.\left\{\bar{p}_{m}^{\mathrm{S}^{n-m}}\right\}_{m=2, \cdots, n-1}\right), \forall n \in \mathcal{N} \backslash\{1\}$, and claim $D\left(p_{n}^{\prime}-\underline{q}_{n}\right) \geq 0$. Basically, $p_{n}^{\prime}$ is composed by some shooting segments from $\left\{\bar{p}_{m}^{\mathrm{s}^{n-m}}\right\}_{m=2, \cdots, n-1}$ and intermediate tangent merging segments. Apparently, $D\left(\bar{p}_{m}^{\mathrm{S}^{n-m}}-\underline{q}_{n}\right) \geq$ 0 . Note that every merging segment shall be above $\underline{p}_{n}$ or it will not catch up with the next shooting segment that accelerates at the maximum rate $\bar{a}^{\mathrm{f}}$ before reaching speed $\bar{v}$. Thus this claim holds and thus $D\left(p_{n}^{\prime}-\bar{q}_{n}\right) \geq-0.5 \bar{v}^{2} / \bar{a}^{\mathrm{f}}$. Therefore, $D\left(p_{n}^{\prime}-q_{n}\right) \geq-0.5 \bar{v}^{2} / \bar{a}^{\mathrm{f}}$. Note that $p_{n}$ is obtained by merging $p_{n}^{\prime}$ and $\bar{p}_{1}^{\mathrm{S}^{n-1}}$ with a tangent segment denoted by $\hat{s}_{n}$. Apparently, $D\left(\bar{p}_{1}^{\mathrm{s}^{n-1}}-q_{n}\right) \geq 0$ since $q_{n}(t)$ is defined as $\min \left\{\bar{q}_{n}(t), p_{1}^{\mathrm{s}^{n-1}}(t)\right\}$. Further note that $D\left(\hat{s}_{n}-\bar{p}_{1}^{\mathrm{s}^{n-1}}\right) \geq 0.5 \bar{v}^{2} / \underline{a}^{\mathrm{f}}$, and since $D\left(\bar{p}_{1}^{\mathrm{s}^{n-1}}-q_{n}\right) \geq 0$, we obtain $D\left(\hat{s}_{n}-q_{n}\right) \geq 0.5 \bar{v}^{2} / \underline{a}^{\mathrm{f}}$. Therefore $D\left(p_{n}-q_{n}\right) \geq \min \left\{D\left(p_{n}^{\prime}-q_{n}\right), D\left(\bar{p}_{1}^{\mathrm{s}^{n-1}}-q_{n}\right), D\left(\hat{s}_{n}-q_{n}\right)\right\} \geq$ $\min \left\{-0.5 \bar{v}^{2} / \bar{a}^{\mathrm{f}}, 0.5 \bar{v}^{2} / \underline{a}^{\mathrm{f}}\right\}$. This completes the proof.

The above theorem reveals that a trajectory generated from $P^{\mathrm{LVP}}\left(\underline{a}^{\mathrm{f}}, \bar{a}^{\mathrm{f}}\right)$ should be always below the counterpart trajectory in $Q$ and the difference is attributed to smoothed accelerations instead of speed jumps. One elegant finding from this theorem is that this difference does not accumulate much across vehicles but is bounded by a constant. Further, the lower bound to this difference is actually tight and we can find instances where $D\left(p_{n}-q_{n}\right)$ for every vehicle $n \in \mathcal{N} \backslash\{1\}$ is exactly identical to $\min \left\{-0.5 \bar{v}^{2} / \bar{a}^{\mathrm{f}}, 0.5 \bar{v}^{2} / \underline{a}^{\mathrm{f}}\right\}$. One such instance can be specified by setting $p_{1}(t)=L, \forall t$ for a sufficiently long $L$, and $v_{n}^{-}=0, \forall n \in \mathcal{N} \backslash\{1\}$. This theorem also leads to the following asymptotic relationship.

Corollary 5. If $\bar{a} f \rightarrow \infty$ and $\underline{a}^{f} \rightarrow-\infty$, theKWTn we have $P^{L V P}\left(\underline{a}^{f}, \bar{a}^{f}\right) \rightarrow Q$.

This relationship indicates that SHL can be viewed as a generalization of Newell's model as well as other equivalent models, including Newell's lower order model and the linear cellular automata model. Essentially, the SH solution can be viewed as a smoothed version of these classic models that circumvents a common problem of these classic models, i.e., infinite acceleration/deceleration or "speed jumps". Such speed jumps would cause unrealistic evaluation of traffic performance measures, particularly those on safety and environmental impacts. Further, SHL inherits the simple structure of these models and thus can be solved efficiently. One commonality between SHL and Newell's solution is that at stationary states, i.e., when each trajectory moves at a constant speed, both the SHL solution and Newell's solution are consistent with a triangular fundamental diagram Newell 1993, as stated below.

Theorem 6. If solutions $P^{L V P}\left(\underline{a}^{f}, \bar{a}^{f}\right)$ and $Q$ are both feasible and each $\dot{p}_{n}(t)$ or $\dot{q}_{n}(t)$ remains constant $\forall t \geq t_{n}^{-}$, then there exists some $V \in[0, \bar{v}]$ such that 


$$
p_{n}(t)=q_{n}(t)=V\left(t-t_{n}^{-}\right), \dot{p}_{n}(t)=\dot{q}_{n}(t)=V, \forall t \in\left[t_{n}^{-}, \infty\right), n \in \mathcal{N}
$$

Further, if $V=\bar{v}$,

$$
t_{n}^{-}-t_{n-1}^{-} \geq \tau+V / s, \forall n \in \mathcal{N}
$$

If $V<\bar{v}$, then

$$
t_{n}^{-}=(n-1)(\tau+V / s)-t_{1}^{-}, \forall n \in \mathcal{N} \backslash\{1\} .
$$

Further, define the traffic density as $K:=\frac{N-1}{\sum_{n=2}^{N}\left(p_{n-1}(t)-p_{n}(t)\right)}=\frac{N-1}{\sum_{n=2}^{N}\left(q_{n-1}(t)-q_{n}(t)\right)}$ for any $t \geq t_{n}^{-}$and traffic volume as $O:=\frac{N-1}{p_{N}^{-1}(l)-p_{1}^{-1}(l)}=\frac{N-1}{q_{N}^{-1}(l)-q_{1}^{-1}(l)}$ for any $l \geq 0$, then we will always have

$$
\begin{gathered}
\begin{cases}K \leq 1 /(s+V \tau), & \text { if } V=\bar{v} ; \\
K=1 /(s+V \tau) & \text { if } V<\bar{v} .\end{cases} \\
O=K V=\min \{K \bar{v},(1-s K) / \tau\} \in[0, \bar{v} /(s+\bar{v} \tau)] .
\end{gathered}
$$

Proof. We fist investigate the case when every $\dot{p}_{n}(t)$ is constant. If there exist two consecutive trajectories not parallel with each other, then they will either intersect or depart from each other to an infinite spacing since they both are straight lines. The former is impossible because of safety constraints (4). Neither is the latter possible since the following trajectory has to accelerate when their spacing exceeds the shadow spacing and runs at a speed lower than the preceding vehicle. Therefore $\dot{p}_{n}(t)$ values are identical to a $V \in[0, \bar{v}]$ across $n \in \mathcal{N}$. Therefore, $p_{n}(t)=V\left(t-t_{n}^{-}\right), \dot{p}_{n}(t)=V, \forall t \in\left[t_{n}^{-}, \infty\right), n \in \mathcal{N}$.

Since $P^{\operatorname{LVP}}\left(\underline{a}^{\mathrm{f}}, \bar{a}^{\mathrm{f}}\right)$ is feasible, we know from Theorem 1 that $\left[v_{n}^{-}, t_{n}^{-}\right]_{n \in \mathcal{N}}$ is proper, indicating that equation (15) holds either $V=\bar{v}$ or $V<\bar{v}$. Further, when $V<\bar{v}$, equation (15) becomes a strict equality (or otherwise the following trajectory needs to accelerate). Thus equation (16) holds in this case.

Then we will show that $q_{n}=p_{n}$. Since all trajectories in $P^{\mathrm{LVP}}\left(\underline{a}^{\mathrm{f}}, \bar{a}^{\mathrm{f}}\right)$ are at speed $V$, we know that $v_{n}^{-}=V, \forall n \in \mathcal{N}$ and the lead trajectory is given as $p_{1}(t)=V\left(t-t_{1}^{-}\right)$. If $V=\bar{v}$, apparently no trajectory blocks its following trajectory, and thus $q_{n}(t)=V\left(t-t_{n}^{-}\right)=p_{n}(t), \forall t \in\left[t_{n}^{-}, \infty\right), n \in \mathcal{N}$. Otherwise if $V<\bar{v}$, due to equation (16), $q_{n}$ has no room to accelerate and thus has to stay at speed $V$. Therefore, equation (14) holds. Then we will prove equations (17) and (18), which quantify the triangular fundamental diagram. First, since $\left[v_{n}^{-}, t_{n}^{-}\right]_{n \in \mathcal{N}}$ is proper, then we obtain $p_{n-1}\left(t_{n}^{-}\right)-p_{n}\left(t_{n}^{-}\right) \geq V \tau+s, \forall n \in \mathcal{N} \backslash\{1\}$, and thus $K \geq 1 /(V \tau+s)$ holds. When $V<\bar{v}$, due to (16), then $p_{n-1}\left(t_{n}^{-}\right)-p_{n}\left(t_{n}^{-}\right)=V \tau+s, \forall n \in$ $\mathcal{N} \backslash\{1\}$, and thus $K=1 /(V \tau+s)$ holds. This proves equation (17). Further note that $p_{n}^{-1}(0)-p_{n-1}^{-1}(0)=$ $\left(p_{n-1}\left(t_{n}^{-}\right)-p_{n}\left(t_{n}^{-}\right)\right) / V, \forall n \in \mathcal{N} \backslash\{1\}$, then $O=\frac{N-1}{\sum_{n=2}^{N}\left(p_{n}^{-1}(0)-p_{n-1}^{-1}(0)\right)}=\frac{N-1}{\sum_{n=2}^{N}\left(p_{n-1}\left(t_{n}^{-}\right)-p_{n}\left(t_{n}^{-}\right)\right) / V}=K V$. When $V=\bar{v}$, plugging inequality $K \leq 1 /(s+V \tau)$ in equation (17) into the previous equation, we obtain $K V=K \bar{v} \leq \bar{v} /(s+\bar{v} \tau)$, and $K \leq 1 /(s+V \tau)$ can be rearranged into to $(1-s K) / \tau \geq K V$. Therefore, equation (18) holds in this case. When $V<\bar{v}$, apparently, $K V<K \bar{v}$. Further, equality $K=1 /(s+V \tau)$ in equation (17) can be rearranged as $K V=(1-s K) / \tau$. Note that $K=1 /(s+V \tau)>1 /(s+\bar{v} \tau)$, and plugging this inequality into $K V=(1-s K) / \tau$ yields $K V=(1-s K) / \tau<(1-s /(s+\bar{v} \tau)) / \tau=\bar{v} /(s+\bar{v} \tau)$. This indicates that equation (18) holds in the case too. This completes the proof.

\section{$5 \quad$ Illustrative Examples}

This section presents a few illustrative examples that help visualize results of the algorithms and related theoretical properties. In the following examples, we set the parameters to their default values unless stated otherwise. These default parameter values are $L=1000 \mathrm{~m}, N=50, \underline{a}=-5 \mathrm{~m} / \mathrm{s}^{2}, \bar{a}=2 \mathrm{~m} / \mathrm{s}^{2}, \bar{v}=25 \mathrm{~m} / \mathrm{s}$, 
$G=R=25 \mathrm{~s}, s=7 \mathrm{~m}$, and the default boundary condition is generated as follows. We first set the arrival times as

$$
t_{1}^{-}=0, t_{n}^{-}=t_{n-1}^{-}+(\tau+s / \bar{v})\left[1+\left(\frac{C}{G f^{\mathrm{S}}}-1\right)\left(1-\alpha+\alpha \epsilon_{n}\right)\right], \forall n \in \mathcal{N} \backslash\{1\},
$$

where $f^{\mathrm{S}} \in(0, C / G]$ is the traffic saturation rate (or the ratio of the arrival traffic volume to the intersection's maximum capacity), $\alpha \in[0,1]$ is the dispersion factor of the headway distribution, and $\left\{\epsilon_{n}\right\}_{n \in \mathcal{N} \backslash\{1\}}$ are uniformly distributed non-negative random numbers that satisfy $\sum_{n \in \mathcal{N} \backslash\{1\}} \epsilon_{n}=N-1$. The default values of $f^{\mathrm{S}}$ and $\alpha$ are both set to 1 . We set the arrival times in this way such that the average time headway equals $(\tau+s / \bar{v}) C /\left(G f^{\mathrm{S}}\right)$ yet the individual arrival times can be stochastic. The stochasticity of the arrival times increases with headway dispersion factor $\alpha$. Note that we always maintain every headway no less than $(\tau+s / \bar{v})$ because the boundary condition would be infeasible otherwise. Next, for each $n \in \mathcal{N}$, the initial speed $v_{n}^{-}$is consecutively drawn as a random number uniformly distributed over the corresponding lower bound and upper bound. These two bounds assure that $v_{n}^{-}$satisfies (i) $v_{n}^{-} \in[(1-\beta) \bar{v}, \bar{v}]$ where factor $\beta \in[0,1]$ controls the dispersion of initial velocities, (ii) $D\left(\bar{p}_{(m s-n s) v_{m}^{-}\left[t_{m}^{-}+(n-m) \tau\right.}-\underline{p}_{0 v_{n}^{-} t_{n}^{-}}\right) \geq 0, \forall m<n \in \mathcal{N}$ and $D\left(\bar{p}_{(n s-m s) v_{n}^{-}\left[t_{n}^{-}+(m-n) \tau\right]}-\underline{p}_{0 v_{m}^{-} t_{m}^{-}}\right) \geq 0, \exists v_{m}^{-} \in[0, \bar{v}], \forall m>n \in \mathcal{N}$ with the maximum acceleration and the minimum deceleration for QTG downscaled to $\bar{a} / 3$ and $\underline{a} / 3$, respectively. The downscale of the acceleration limits assures that the boundary condition is feasible for any forward acceleration $\bar{a}^{\mathrm{f}} \geq \bar{a} / 3$ and any forward deceleration $\underline{a}^{\mathrm{f}} \geq \underline{a} / 3$, which allows to test a range of $\bar{a}^{\mathrm{f}}$ and $\underline{a}^{\mathrm{f}}$ values. If a proper boundary condition cannot be found, we will regenerate random parameters $\left\{\epsilon_{n}\right\}_{n \in \mathcal{N} \backslash\{1\}}$ with a different random seed and repeat this process until finding a proper boundary condition. The default value of $\beta$ is set to 0.5 .

Section 5.1 compares the SH solutions with a benchmark instance that simulates the manually-driven traffic counterpart. This comparison aims to qualitatively show the advantages of the proposed CAV control strategies over manual driving. Section 5.2 compares SHL and PSHL results and shows they produce identical results for the same LVP input. Section 5.3 tests the feasibility of SH and SHL solutions with different boundary conditions to verify some theory predictions and reveal insights into how parameter changes affect the solution feasibility. Section 5.4 compares SHL and LWK results and measures their difference to check the theoretical error bounds.

\subsection{Manual v.s. Automated Trajectories}

We construct a benchmark instance that simulates the manually-driven traffic counterpart by adapting Gipp's Model (Gipps, 1981) as the manual-driving rule for every vehicle:

$$
\ddot{p}_{n}(t)=\min \left\{\bar{a} \mathbf{I}\left(\dot{p}_{n}(t)<\bar{v}\right), a_{n}^{\mathrm{safe}}(t) \mathbf{I}\left(\dot{p}_{n}(t)>0\right)\right\},
$$

where indicator function $\mathbf{I}(\cdot)$ is 1 if condition - holds or 0 otherwise, and safety acceleration $a_{n}^{\text {safe }}$ is defined as follows:

$$
a_{n}^{\text {safe }}(t)= \begin{cases}\infty, & \text { if } \dot{p}_{n}(t)^{2} / b<s_{n}^{\text {safe }}(t) ; \\ \ddot{p}_{n-1}(t-\tau), & \text { if } \dot{p}_{n}(t)^{2} / b=s_{n}^{\text {safe }}(t) ; \\ -b, & \text { if } \dot{p}_{n}(t)^{2} / b>s_{n}^{\text {safe }}(t),\end{cases}
$$

where comfort deceleration $b=\underline{a} / 2$, and safety distance function $s_{n}^{\mathrm{Safe}}(t)$ is determined by the following equation, depending on whether vehicle $n$ is or will be blocked by a red light:

$$
s_{n}^{\mathrm{safe}}(t)= \begin{cases}s_{n}^{\mathrm{F}}(t), & \text { if } \bar{p}_{p_{n}(t) \dot{p}_{n}(t) t}^{-1}(L) \in \mathcal{G} \text { and } t \in \mathcal{G} \\ \min \left\{s_{n}^{\mathrm{F}}(t), s_{n}^{\mathrm{R}}(t)\right\}, & \text { otherwise, }\end{cases}
$$

where the safety distance due to car following is $s_{n}^{\mathrm{F}}(t):=p_{n-1}(t-\tau)-s-p_{n}(t)+\dot{p}_{n}(t-\tau)^{2} / b$, and the safety distance due to red light blocking is $s_{n}^{\mathrm{R}}(t):=L-p_{n}(t)$. To accommodate the lead vehicle that does not have 


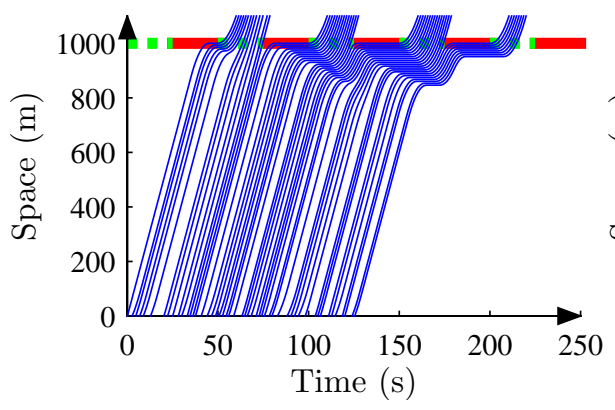

(a)

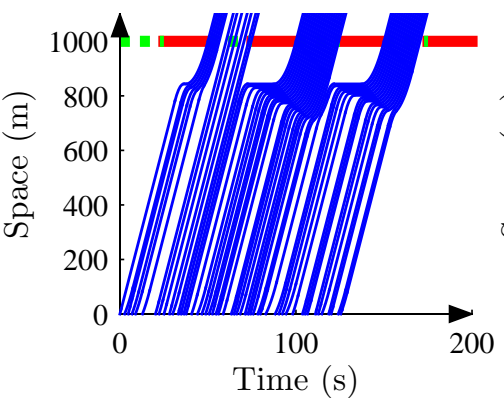

(b)

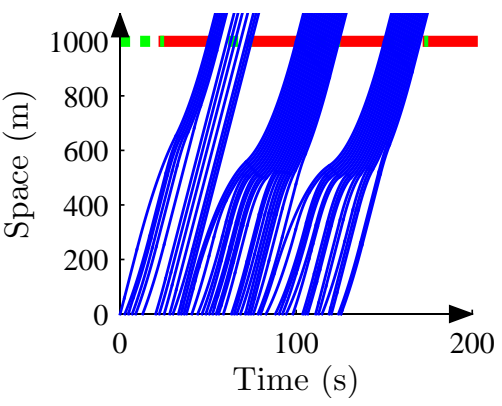

(c)

Figure 7: (a) Benchmark manual-driving trajectories, (b) SH result $P(\bar{a}, \underline{a}, \bar{a}, \underline{a})$, and (c) $\mathrm{SH}$ result $P(\bar{a} / 3, \underline{a} / 3, \bar{a} / 3, \underline{a} / 9)$.

a preceding trajectory, without loss of generality, we define a dummy vehicle 0 with $p_{0}(t)=\infty, \dot{p}_{0}(t)=\infty$, and $\ddot{p}_{0}(t)=-b, \forall t$. Note that the above model is a continuous model, and coding it on a computer requires proper discretization. There are two reasons for selecting this model. First, the trajectories produced from this model appear to be consistent with our driving experience at signalized intersections: we tend to slow down and make a stop only when we get close to the intersection during a yellow or red light. Second, it is easy to verify that at a stationary state, the same macroscopic relationship between density and volume defined in Theorem 6 holds. This way, the comparison between the SH solution and this benchmark will only focus on the "trajectory smoothing" effect rather than improvement of stationary traffic characteristics, which has been investigated in other studies (e.g., Shladover et al. (2009)).

Figure 7 compares the benchmark result with the SH output. Figure 7(a) plots the benchmark manualdriving trajectories generated with car-following model (20). We see that due to abrupt accelerations and decelerations in the vicinity of the traffic lights, a number of consecutive stop-and-go waves are formed and propagated backwards from the intersection. These stop-and-go waves slow down the passing speed of the vehicles at the intersections, and thus decrease the traffic throughput and increase the travel delay. As a result, the total travel time (i.e., the time duration between the first vehicle's entry at location 0 and the last vehicle's exist at location $L$ ) is over 215 seconds for the benchmark case. Further, it is intuitive that these stop-and-go waves adversely impact fuel consumptions and emissions and amplify collision risks. Figure 7(b) plots the SH result with $\left(\bar{a}^{\mathrm{f}}, \underline{a}^{\mathrm{f}}, \bar{a}^{\mathrm{b}}, \underline{a}^{\mathrm{b}}\right)$ identical to their bounding values $(\bar{a}, \underline{a}, \bar{a}, \underline{a})$. We see that despite some sharp accelerations and decelerations, all vehicles can pass the intersection at the maximum speed and thus the traffic throughput gets maximized. The total travel time now is only around 170 seconds. Figure $7(\mathrm{c})$ plots the SH result with $\left(\bar{a}^{\mathrm{f}}, \underline{a}^{\mathrm{f}}, \bar{a}^{\mathrm{b}}, \underline{a}^{\mathrm{b}}\right)$ downscaled to $(\bar{a} / 3, \underline{a} / 3, \bar{a} / 3, \underline{a} / 9)$. We see that with the acceleration/deceleration magnitudes reduced, trajectories become much smoother while the total travel time remains low around 170 seconds. This will further reduce the traffic's environmental impacts and enhance its safety.

To inspect the differences between the benchmark and the SH results from a macroscopic point of view, we measure the macroscopic traffic characteristics, including density and flow volumes for the trajectory sets in Figure 7 with the measuring method proposed by Laval (2011b). Basically, we roll a parallelogram with a length of $100 \mathrm{~m}$ and a time interval of $5 \mathrm{~s}$ along the shock wave direction (at a speed of $-s / \tau$ ) across the trajectories in every plot in Figure 7 by a $100 \mathrm{~m} \times 5 \mathrm{~s}$ step size. We measure the flow volume and density at each parallelogram and plot the measurements as circles in the corresponding diagrams in Figure 8, where the solid curves represent the stationary flow-density relationships specified in equation (18). We see that in Figure 8(a) for the bench mark trajectories, many measurements are distributed on the congested side of this diagram and most of them are below the stationary curve. This explains why the performance of the benchmark case is the worst. This is probably because the stop and go waves (or traffic oscillations) result in a lower traffic throughput even at the same density, which is known as the capacity drop phenomenon (Cassidy and Bertini, 1999; Ma et al., 2015). In Figure 8(b) for the SH result $P(\bar{a}, \underline{a}, \bar{a}, \underline{a})$, there are fewer 


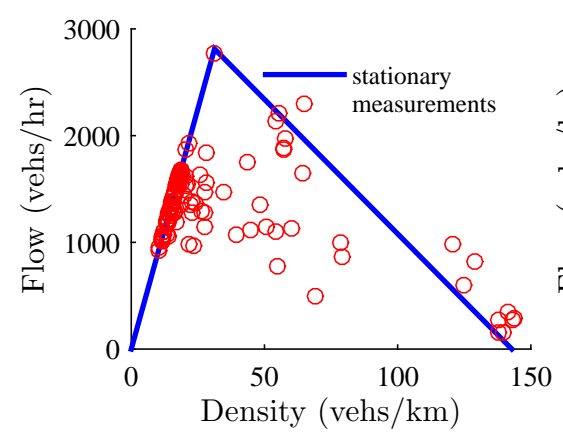

(a)

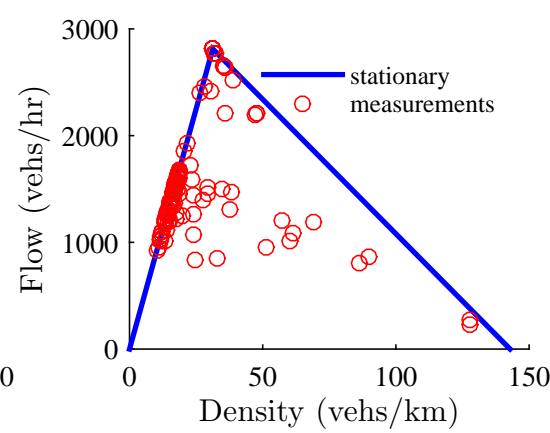

(b)

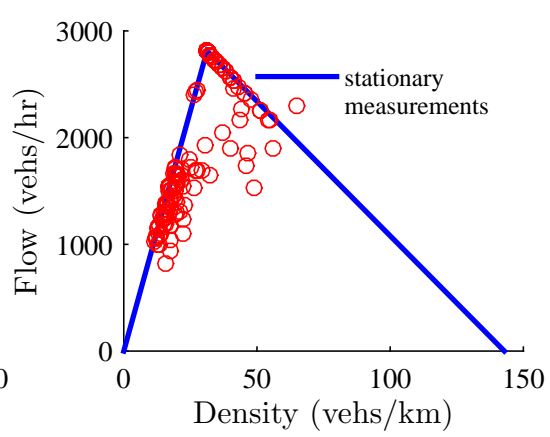

(c)

Figure 8: Macroscopic characteristics for (a) the benchmark trajectories, (b) $P(\bar{a}, \underline{a}, \bar{a}, \underline{a})$, and (c) $P(\bar{a} / 3, \underline{a} / 3, \bar{a} / 3, \underline{a} / 9)$.

measurements falling in the congested branch, and these measurements are closer to the stationary curve. In Figure 8(c) for the smoothed SH result $P(\bar{a} / 3, \underline{a} / 3, \bar{a} / 3, \underline{a} / 9)$, much more measurements lie in the freeflow branch, and these measurements become consistent with the stationary curve. This suggests that the proposed SH algorithm with proper parameter values can counteract the capacity drop phenomenon and bring macroscopic traffic characteristics toward the free-flow branch of the stationary curve.

Overall, the results in this Subsection show that the proposed SH algorithm can significantly improve the highway traffic performance in terms of mobility, environment and safety. To realize the full utility of the SH algorithm, quantitative optimization needs to be conducted, which will be detailed in Part II of this study.

\subsection{Lead Vehicle Problem}

This subsection presents LVP results from manual driving law (20) and the proposed CAV driving algorithms. In the LVP, we set $G=\infty$ and $R=0$, and we update saturation rate to $f_{s}=0.5$ in generating the boundary condition (so that the average headway remains $0.5(\tau+s / \bar{v})$ ). The lead trajectory is set to initially cruise at speed $\bar{v}$ for 20 seconds, then deceleration to the zero speed with a decelerating rate of $\underline{a} / 3$, then keep stopped for 20 seconds, then accelerate to $\bar{v}$ with a rate of $\bar{a} / 3$, and finally keep cruising at this speed, i.e.,

$$
\begin{aligned}
p_{1}:= & {\left[(0, \bar{v}, 0,0,20),\left(20 \bar{v}, \bar{v}, \frac{a}{3}, 20,20-\frac{3 \bar{v}}{\underline{a}}\right),\left(20 \bar{v}-\frac{3 \bar{v}^{2}}{2 \underline{a}}, 0,0,20-\frac{3 \bar{v}}{\underline{a}}, 40-\frac{3 \bar{v}}{\underline{a}}\right),\right.} \\
& \left.\left(20 \bar{v}-\frac{3 \bar{v}^{2}}{2 \underline{a}}, 0, \frac{a}{3}, 40-\frac{3 \bar{v}}{\underline{a}}, 40-\frac{3 \bar{v}}{\underline{a}}+\frac{3 \bar{v}}{\bar{a}}\right),\left(20 \bar{v}-\frac{3 \bar{v}^{2}}{2 \underline{a}}+\frac{3 \bar{v}^{2}}{2 \bar{a}}, \bar{v}, 0,40-\frac{3 \bar{v}}{\underline{a}}+\frac{3 \bar{v}}{\bar{a}}, \infty\right)\right] .
\end{aligned}
$$

This way, $p_{1}$ triggers a stopping wave and we can examine its propagation under different driving conditions. Figure 9 shows the trajectory comparison results. We see that first, in Figures 9 (b) and (c), the PSHL results (solid lines) exactly overlap with the SHL results (crosses), which verifies Proposition 2 that states the equivalence between PSHL and SHL. Compared with the manual-driving case in Figure 9(a), the automateddriving case in Figure 9(b), even with the extreme acceleration and deceleration rates $(\bar{a}, \underline{a})$, relatively better absorbs the backward stopping wave within a fewer number of vehicles. Reducing the acceleration and deceleration magnitudes to $(\bar{a} / 3, \underline{a} / 3)$ in Figure $9(\mathrm{c})$ can further smooth vehicle trajectories and dampen the impact from the stopping wave. These results imply that proper CAV controls can effectively smooth stop-and-go traffic and reduce backward shock wave propagation on a freeway. 


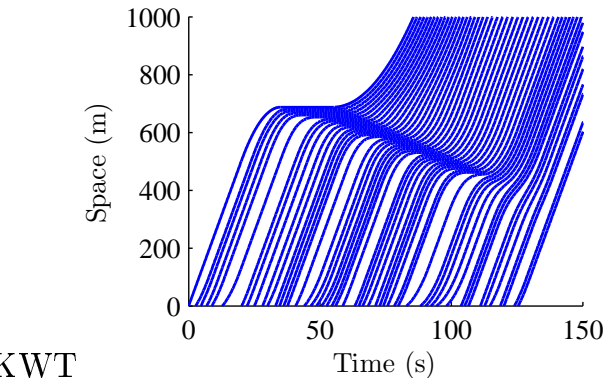

(a)

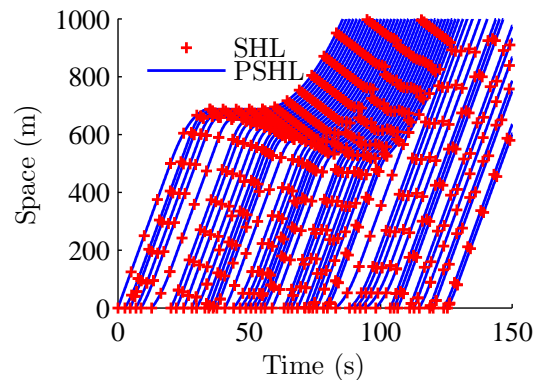

(b)

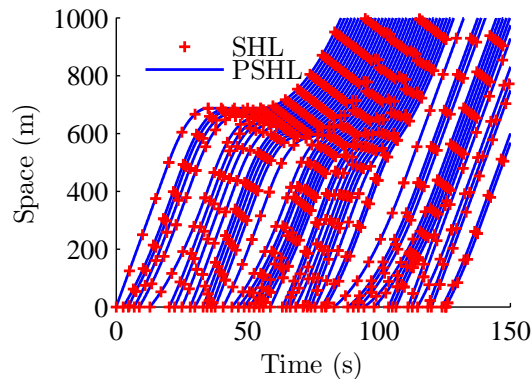

(c)

Figure 9: (a) Benchmark manual-driving trajectories, (b) SHL and PSHL results with $\left(\bar{a}^{\mathrm{f}}, \underline{a}^{\mathrm{f}}\right)=(\bar{a}, \underline{a})$, and (c) SHL and PSHL results with $\left(\bar{a}^{\mathrm{f}}, \underline{a}^{\mathrm{f}}\right)=(\bar{a} / 3, \underline{a} / 3)$.

\subsection{Feasibility Tests}

This section conducts some numerical tests to test the feasibility of the proposed algorithms with different input settings. We first investigate SHL (or PSHL) for LVP with $\left(\bar{a}^{\mathrm{f}}, \underline{a}^{\mathrm{f}}\right)=(\bar{a}, \underline{a})$. Theorem 1 proves that SHL is feasible if and only if boundary condition $\left[v_{n}^{-}, t_{n}^{-}\right]_{n \in \mathcal{N}}$ is proper. Thus we investigate how the feasibility of SHL changes with the distribution of $v_{n}^{-}$and $t_{n}^{-}$. Since the default boundary condition generation method always assures that $\left[v_{n}^{-}, t_{n}^{-}\right]_{n \in \mathcal{N}}$ is proper, this subsection uses a different generation method to allow $\left[v_{n}^{-}, t_{n}^{-}\right]_{n \in \mathcal{N}}$ to be non-proper. We still use equation (19) to generate $t_{n}^{-}$, and thus the dispersion of time headway is controlled by $\alpha$. In the next step, each $v_{n}^{-}$is instead randomly pulled along a uniform interval $[(1-\beta) \bar{v}, \bar{v}]$ without checking the boundary condition properness. Note that the final values of $t_{n}^{-}$and $v_{n}^{-}$are randomly generated. We generate 20 boundary condition instances with the same $\alpha$ and $\beta$ values yet different random seeds. Then we feed each boundary condition instance to the SHL algorithm and record the feasibility of the result. We call the percentage of feasible solutions over all 20 boundary conditions the feasibility rate with regard to this specific parameter setting. Figure 10 plots hot maps for the feasibility rate over $\alpha \times \beta \in[0,1] \times[0,1]$ with different $f^{\mathrm{S}}$ values. We can see that overall, as $\alpha$ and $\beta$ increase, the feasibility rate decreases, and more instances are infeasible as $f^{\mathrm{S}}$ increases. This is because higher dispersion of $t_{n}^{-}$and $v_{n}^{-}$is more likely to cause conflicts between trajectories that cannot be reconciled under safety constraint (4), and such conflicts may increase as traffic gets denser. Note that in all maps in Figure 10, the transition band between $100 \%$ feasibility rate (the white color) and $0 \%$ feasibility rate (the black color) is very narrow. This indicates that SHL's feasibility is dichotomous. With this observation, the parameters could be partitioned into only two phases (feasible and infeasible) to facilitate relevant analysis.

Next we investigate the $\mathrm{SH}$ algorithm considering traffic lights with $\left(\bar{a}^{\mathrm{f}}, \underline{a}^{\mathrm{f}}, \bar{a}^{\mathrm{b}}, \underline{a}^{\mathrm{b}}\right)=(\bar{a}, \underline{a}, \bar{a}, \underline{a})$. We conduct similar experiments as those for Figure 10 with the same adapted boundary condition generation method. Theorems 2 and 3 suggest that the feasibility of SH is related to segment length $L$. Traffic congestion $f^{\mathrm{S}}$ and platoon size $N$ shall also affect SH's feasibility. This time, we fix $\alpha=\beta=0.5$ and analyze how the feasibility rate varies with $L$ and $f^{\mathrm{S}}$ over different $N$ values, and the results are shown in Figure 11. We see that again the increase of $f^{\mathrm{S}}$ raises the chance of infeasibility. Further, as $L$ decreases, the likelihood of feasibility diminishes. This is because a short segment may not be sufficient to store enough stopping or slowly moving vehicles to both comply with the signal phases and allow the following vehicles to enter the segment at their due times. Also, more instances are infeasible as $N$ increases. This is because more vehicles shall bare a higher chance of producing an irreconcilable conflict between two consecutive vehicles against safety constraint (4). Similarly, the SH feasibility is dichotomous and a two-phase representation might be applicable. 


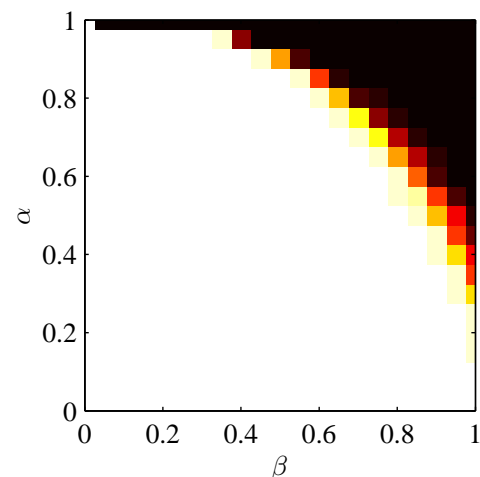

(a)

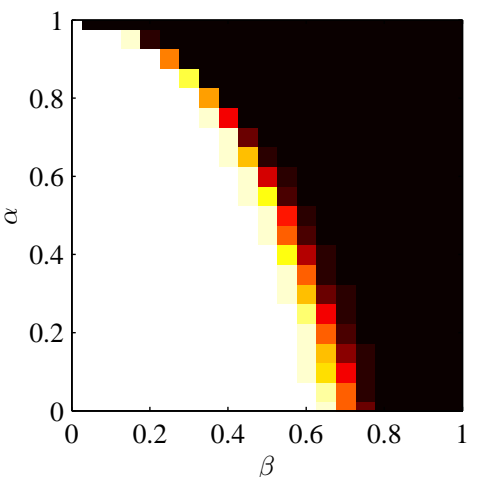

(b)

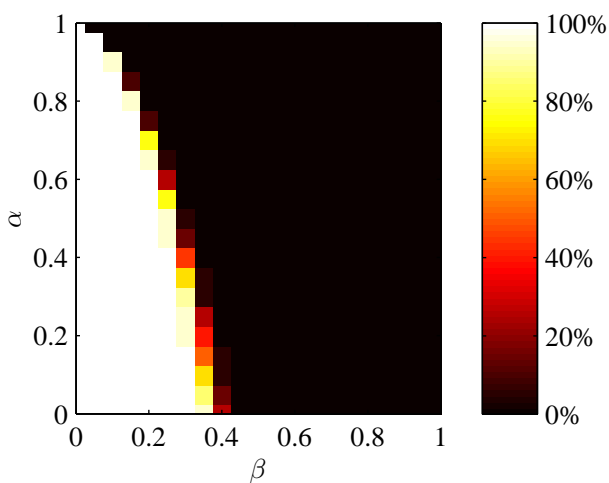

(c)

Figure 10: Feasibility rate of PSHL with (a) $f^{\mathrm{S}}=0.2$, (b) $f^{\mathrm{S}}=0.5$, and (c) $f^{\mathrm{S}}=0.8$.

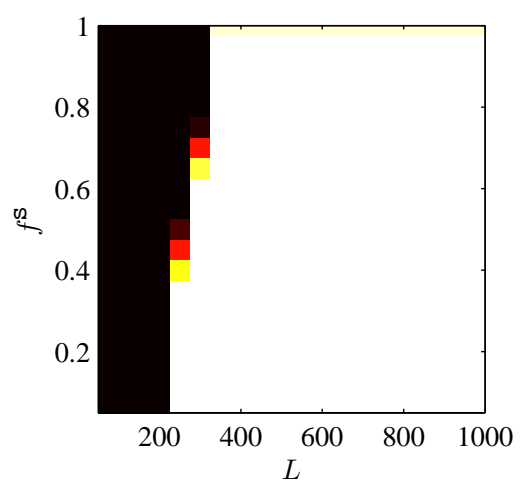

(a)

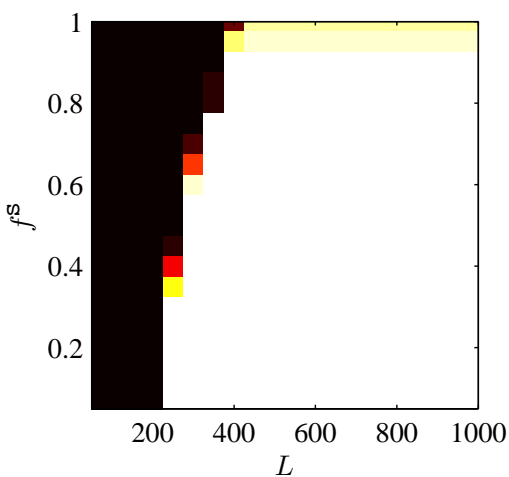

(b)

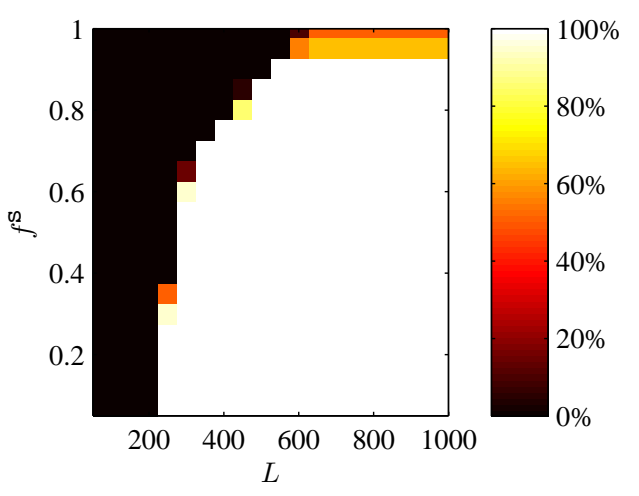

(c)

Figure 11: Feasibility rate of PSHL with (a) $N=25$; (b) $N=50$; and (c) $N=100$. 


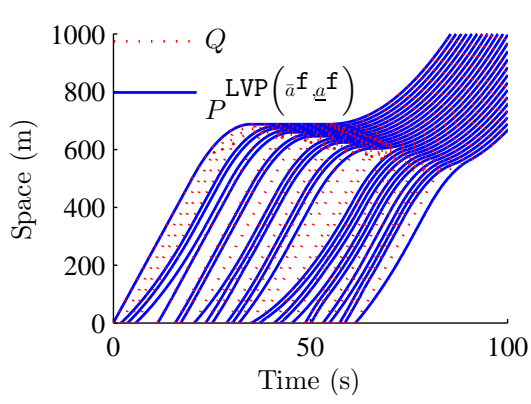

(a)

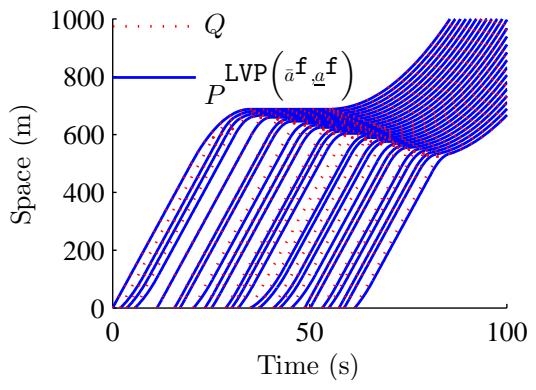

(b)

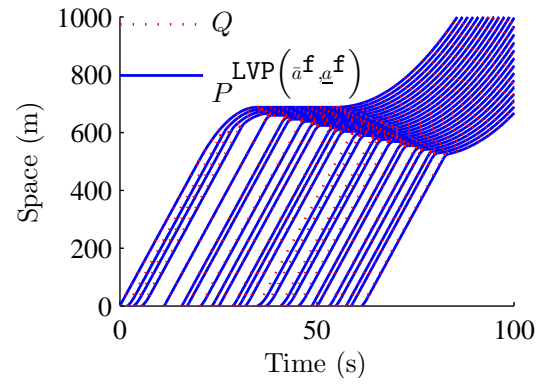

(c)

Figure 12: Comparison between Newell's solution $Q$ and SHL solution $P^{\mathrm{LVP}}\left(\bar{a}^{\mathrm{f}}, \underline{a}^{\mathrm{f}}\right)$ with $(\mathrm{a})\left(\bar{a}^{\mathrm{f}}, \underline{a}^{\mathrm{f}}\right)=$ $(\bar{a} / 3, \underline{a} / 3),(\mathrm{b})\left(\bar{a}^{\mathrm{f}}, \underline{a}^{\mathrm{f}}\right)=(\bar{a}, \underline{a})$, and $(\mathrm{c})\left(\bar{a}^{\mathrm{f}}, \underline{a}^{\mathrm{f}}\right)=(3 \bar{a}, 3 \underline{a}) .$.

\subsection{Comparison with Classic Traffic Flow Models}

This section compares Newell's solution $Q$ and SHL solution $P^{\mathrm{LVP}}\left(\underline{a}^{\mathrm{f}}, \bar{a}^{\mathrm{f}}\right)$ for LVP. Again, we set $G=\infty$ and $R=0, f^{\mathrm{S}}=0.5$. The lead trajectory is generated with equation (21), and the boundary condition is generated with the default method associated with equation (19) to assure the feasibility. Figure 12 compares these trajectories with different $\left(\bar{a}^{\mathrm{f}}, \underline{a}^{\mathrm{f}}\right)$ values. In this section we allow $\left(\bar{a}^{\mathrm{f}}, \underline{a}^{\mathrm{f}}\right)$ to go beyond $(\bar{a}, \underline{a})$ to investigate the asymptotic properties of SHL. We see that in general, trajectories in $Q$ have abrupt turns while those in $P^{\mathrm{LVP}}\left(\underline{a}^{\mathrm{f}}, \bar{a}^{\mathrm{f}}\right)$ are relatively smooth. All trajectories in $P^{\mathrm{LVP}}\left(\underline{a}^{\mathrm{f}}, \bar{a}^{\mathrm{f}}\right)$ is below those in $P^{\mathrm{LVP}}\left(\underline{a}^{\mathrm{f}}, \bar{a}^{\mathrm{f}}\right)$, which is consistent with the upper bound property stated in Theorem 5. As $\left(\bar{a}^{\mathrm{f}}, \underline{a}^{\mathrm{f}}\right)$ amplifies from $(\bar{a} / 3, \underline{a} / 3)$ to $(3 \bar{a}, 3 \underline{a})$, we see that accelerations and decelerations in $P^{\operatorname{LVP}}\left(\underline{a}^{\mathrm{f}}, \bar{a}^{\mathrm{f}}\right)$ become sharper and trajectories in $P^{\mathrm{LVP}}\left(\underline{a}^{\mathrm{f}}, \bar{a}^{\mathrm{f}}\right)$ get closer to those in $Q$, which is consistent with the asymptotic property stated in Corollary 5.

Figure 13 plots the errors between $Q$ and $P^{\mathrm{LVP}}(\gamma \bar{a}, \gamma \underline{a})$ and their bounds (defined in Theorem 5$)$, where acceleration factor $\gamma$ increases from $1 / 3$ to 3 . We see that $D\left(q_{n}-p_{n}\right)$ is always identical to 0 (or the upper bound) and $D\left(p_{n}-q_{n}\right)$ is always above the lower bound (13) for all $\gamma$ values. As $\gamma$ increases, the errors and their bounds all converge to 0 , which again confirms Corollary 5 . In summary, these experiments show that SHL can be viewed as a smoothed version of Newell's solution that replaces speed jumps in Newell's solution with smooth accelerations and decelerations.

\section{Conclusion}

This paper investigates the problem of controlling multiple vehicle trajectories on a highway with CAV technologies. We propose a shooting heuristic to efficiently construct vehicle trajectories that follow one another under a number of constraints, including the boundary conditions, physical limits, following safety, and traffic signals. With slight adaptation, this heuristic is applicable to not only highway arterials with interrupted traffic but also uninterrupted freeway traffic. We generalize the time geography theory to consider finite accelerations. This allows us to study the behavior of the proposed algorithms. We find that the proposed algorithms can always find a feasible solution to the original complex multi-trajectory design problem under certain mild conditions. We further point out that the shooting heuristic solution to the lead vehicle problem can be viewed as a smoothed version of the classic kinematic theory's result. We find that the kinematic wave theory is essentially a special case of the proposed shooting heuristic with infinite accelerations. Further, the difference between the shooting heuristic solution and the kinematic wave 


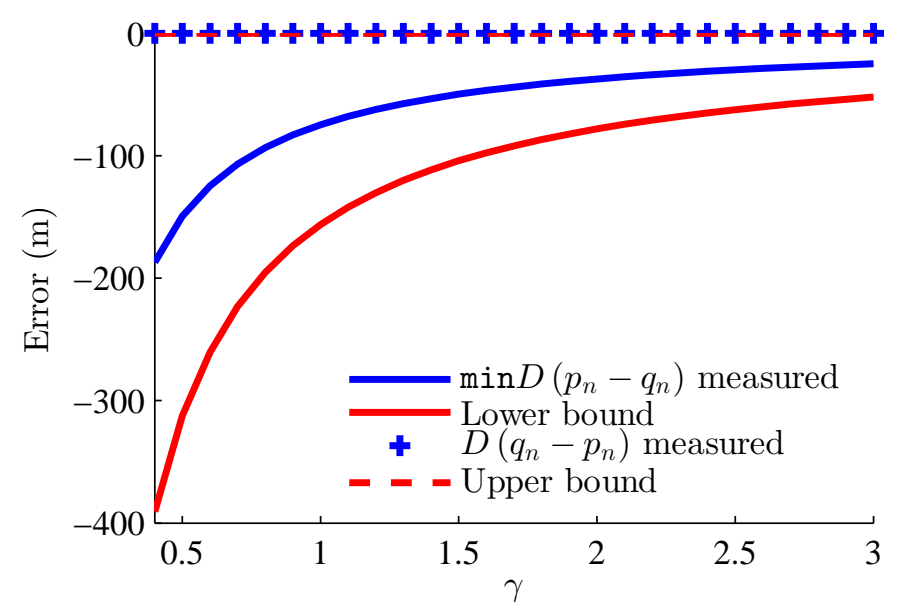

Figure 13: Measured errors between Newell's solution $Q$ and SHL solution $P^{\mathrm{LVP}}(\gamma \bar{a}, \gamma \underline{a})$ v.s. their bounds

solution is found to be limited within two theoretical bounds independent of the size of the vehicles in the studied traffic stream. Numerical experiments are conducted to illustrate some theoretical results and draw additional insights into how the proposed algorithms can improve highway traffic.

This paper provides a methodological and theoretical foundation for managing future CAV traffic. The following part II paper Ma et al. (2015) of this study will apply the proposed shooting heuristic with given acceleration rates to a trajectory optimization framework that optimizes the overall performance of CAV traffic (e.g., in terms of mobility, environment impacts and safety) by finding the best acceleration rates. Theoretical results and numerical examples on this optimization framework will be presented. This study overall expects to provide both theoretical principles and application guidance for upgrading the existing highway traffic management systems with emerging CAV technologies. It can be extended in a number of directions to address practical challenges and emerging opportunities in deploying CAV technologies. While the proposed method that generates vehicle trajectories with continuous speeds makes significant improvement over those allowing for speed jumps, the output trajectories still have acceleration jumps. This can be overcome by replacing or connecting current parabolic segments with smoother curves, e.g., third-order curves, spiral curves, adapted logistic function curves, etc. The current SH algorithm assumes a constant acceleration limit $\bar{a}$, which however should be a nonlinear function of vehicle speed. It can be adapted to check the speed dependent acceleration limit on each constructed quadratic trajectory segment separately. The proposed algorithm is essentially a short-term trajectory planning problem. Its applicability will be strengthened if it can be integrated with the real-time feedback control to ensure that vehicles properly follow the planned trajectories considering delays and errors in observations and controllers. These single-lane single-segment results can be used as building blocks to investigate more complex geometries, e.g., multisegment corridors, intersections, multi-lane highways, and even a complete network. It is also interesting to investigate heterogeneous characteristics of vehicles, e.g., in terms of sizes, acceleration capabilities and energy efficiency. It is also interesting to investigate how heterogeneous destinations rather than pure through traffic would affect the trajectory shapes. While the current study assumes the signal timing is fixed all the time, emerging technologies allow CAVs to negotiate with signals to adjust timing plans in real time. It is very interesting to examine how integrating trajectory control with signal optimization will further improve the system performance. This study assumes that the control scheme is purely centralized, which provides the optimal benchmark for a decentralized system that is probably more likely in the near future. It is worthwhile to investigate how to design information management schemes in such a decentralized system to push the system performance towards this optimal benchmark. If we only control a portion of the trajectories and generate the remaining ones with manual car-following laws, this development can be adapted to investigate 
traffic with mixed manual and automated vehicles. When the hardware is ready, field experiments can be conducted to validate and calibrate the theoretical models.

\section{Acknowledgments}

This research is supported in part by the U.S. National Science Foundation through Grants CMMI CAREER\#1453949, CMMI \#1234936 and CMMI \#1541130. The helpful comments from Dr. Xiaobo Qu, Dr. Yiheng Feng and Dr. Henry Liu are much appreciated.

\section{References}

Ahn, K., Rakha, H., Park, S., 2013. Ecodrive application: Algorithmic development and preliminary testing. Transportation Research Record: Journal of the Transportation Research Board (2341), 1-11.

Ahn, S., 2005. Formation and spatial evolution of traffic oscillations. Ph.D. thesis, University of California, Berkeley, Berkeley, California.

Apostol, T. M., 1969. Calculus, vol. ii. Ed Reverté.

Bando, M., Hasebe, K., Nakanishi, K., Nakayama, A., 1998. Analysis of optimal velocity model with explicit delay. Physical Review E 58 (5), 5429-5435.

Bando, M., Hasebe, K., Nakayama, A., Shibata, A., Sugiyama, Y., 1995. Dynamical model of traffic congestion and numerical simulation. Physical Review E 51 (2), 1035-1042.

Beard, G. F., Griffin, M. J., 2013. Discomfort during lateral acceleration: Influence of seat cushion and backrest. Applied ergonomics 44 (4), 588-594.

Bham, G. H., Long, S., Baik, H., Ryan, T., Gentry, L., Lall, K., Arezoumandi, M., Liu, D., Li, T., Schaeffer, B., 2010. Evaluation of variable speed limits on i-270/i-255 in st. louis. Tech. rep.

Brackstone, M., McDonald, M., 1999. Car-following: a historical review. Transportation Research Part F: Traffic Psychology and Behaviour 2 (4), 181 - 196.

Branston, D., van Zuylen, H., 1978. The estimation of saturation flow, effective green time and passenger car equivalents at traffic signals by multiple linear regression. Transportation Research 12 (1), 47-53.

Cassidy, M., Bertini, R., 1999. Some traffic features at freeway bottlenecks. Transportation Research Part B $33(1), 25-42$.

Chandler, R. E., Herman, R., Montroll, E. W., 1958. Traffic dynamics: Studies in car following. Operations Research 6 (2), 165-184.

Daganzo, C. F., 1994. The cell transmission model: A dynamic representation of highway traffic consistent with the hydrodynamic theory. Transportation Research Part B: Methodological 28 (4), $269-287$.

Daganzo, C. F., 2006. In traffic flow, cellular automata=kinematic waves. Transportation Research Part B: Methodological 40 (5), $396-403$.

Daganzo, C. F., Cassidy, M. J., Bertini, R. L., 1999. Possible explanations of phase transitions in highway traffic. Transportation Research Part A: Policy and Practice 33 (5), 365-379.

De Nunzio, G., Canudas de Wit, C., Moulin, P., Di Domenico, D., 2013. Eco-driving in urban traffic networks using traffic signal information. In: Decision and Control (CDC), 2013 IEEE 52nd Annual Conference on. IEEE, pp. 892-898. 
Dresner, K., Stone, P., 2008. A multiagent approach to autonomous intersection management. Journal of Artificial Intelligence Research 31, 591-656.

Gipps, P. G., 1981. A behavioural car-following model for computer simulation. Transportation Research Part B 15 (2), 105-111.

Guan, T., Frey, C. W., 2013. Predictive fuel efficiency optimization using traffic light timings and fuel consumption model. In: Intelligent Transportation Systems-(ITSC), 2013 16th International IEEE Conference on. IEEE, pp. 1553-1558.

Hegyi, A., De Schutter, B., Hellendoorn, H., 2005. Model predictive control for optimal coordination of ramp metering and variable speed limits. Transportation Research Part C: Emerging Technologies 13 (3), $185-209$.

Herman, R., Montroll, E. W., Potts, R. B., Rothery, R. W., 1958. Traffic dynamics: Analysis of stability in car following. Operations Research 7 (1), 86-106.

Hoffmann, E. R., Mortimer, R. G., 1994. Drivers' estimates of time to collision. Accident Analysis \& Prevention $26(4), 511-520$.

Hooker, J., 1988. Optimal driving for single-vehicle fuel economy. Transportation Research Part A: General $22(3), 183-201$.

Iglesias, I., Isasi, L., Larburu, M., Martinez, V., Molinete, B., 2008. I2v communication driving assistance system: on-board traffic light assistant. In: Vehicular Technology Conference, 2008. VTC 2008-Fall. IEEE 68th. IEEE, pp. 1-5.

Islam, M., Hadiuzzaman, M., Fang, J., Qiu, T., El-Basyouny, K., 2013. Assessing mobility and safety impacts of a variable speed limit control strategy. Transportation Research Record: Journal of the Transportation Research Board (2364), 1-11.

Kamalanathsharma, R. K., Rakha, H., et al., 2013. Multi-stage dynamic programming algorithm for ecospeed control at traffic signalized intersections. In: Intelligent Transportation Systems-(ITSC), 2013 16th International IEEE Conference on. IEEE, pp. 2094-2099.

Kerner, B., 1998. Experimental features of self-organization in traffic flow. Physical Review Letter 81 (17), $3797-3800$.

Kerner, B., Rehborn, H., 1996. Experimental properties of complexity in traffic flow. Physical Review E 53 (5), R4275-R4278.

Kerner, B. S., Rehborn, H., 1997. Experimental properties of phase transitions in traffic flow. Physical Review Letters 79 (20), 4030-4033.

Kuhne, R. D., 1987. Freeway speed distribution and acceleration noise. In: Gartner, N. H., Wilson, N. H. (Eds.), Proceedings of the 10th International Symposium on Transportation and Traffic Theory. pp. 119137.

Kuijpers, B., Miller, H. J., Othman, W., 2011. Kinetic space-time prisms. In: Proceedings of the 19th ACM SIGSPATIAL international conference on advances in geographic information systems. ACM, pp. 162-170.

Laval, J. A., 2011a. Hysteresis in traffic flow revisited: An improved measurement method. Transportation Research Part B 45 (2), 385 - 391.

Laval, J. A., 2011b. Hysteresis in traffic flow revisited: An improved measurement method. Transportation Research Part B 45 (2), 385 - 391. 
Lee, J., Park, B., 2012a. Development and evaluation of a cooperative vehicle intersection control algorithm under the connected vehicles environment. Intelligent Transportation Systems, IEEE Transactions on $13(1), 81-90$.

Lee, J., Park, B., 2012b. Development and evaluation of a cooperative vehicle intersection control algorithm under the connected vehicles environment. Intelligent Transportation Systems, IEEE Transactions on $13(1), 81-90$.

Li, X., Cui, J., An, S., Parsafard, M., 2014. Stop-and-go traffic analysis: Theoretical properties, environmental impacts and oscillation mitigation. Transportation Research Part B: Methodological 70, 319-339.

Li, X., Ouyang, Y., 2011. Characterization of traffic oscillation propagation under nonlinear car-following laws. Transportation Research Part B 45 (9), 1346-1361.

Lighthill, M. J., Whitham, G. B., 1955. On Kinematic Waves. II. A Theory of Traffic Flow on Long Crowded Roads. Royal Society of London Proceedings Series A 229, 317-345.

Liu, B., Ghosal, D., Chuah, C.-N., Zhang, H. M., 2012. Reducing greenhouse effects via fuel consumptionaware variable speed limit (fc-vsl). Vehicular Technology, IEEE Transactions on 61 (1), 111-122.

Lu, X.-Y., Shladover, S., 2014. Review of variable speed limits and advisories: Theory, algorithms, and practice. Transportation Research Record: Journal of the Transportation Research Board (2423), 15-23.

Ma, J., Li, X., Zhou, F., Hu, J., Park, B., 2015. A parsimonious two-way shooting algorithm for connected automated traffic smoothing part ii: Optimization framework. Submitted to Transportation Research Part B [http://dx.doi.org/10.13140/RG.2.1.1721.1924].

Mandava, S., Boriboonsomsin, K., Barth, M., 2009. Arterial velocity planning based on traffic signal information under light traffic conditions. In: Intelligent Transportation Systems, 2009. ITSC'09. 12th International IEEE Conference on. IEEE, pp. 1-6.

Mauch, M., Cassidy, M., 2002. Freeway traffic oscillations: observations and predictions. In: Taylor, M. A. (Ed.), Proceedings of the 15th International Symposium on Transportation and Traffic Theory. pp. 653674 .

Miller, H. J., 2005. A measurement theory for time geography. Geographical analysis 37 (1), 17-45.

Nagel, K., Schreckenberg, M., 1992. A cellular automaton model for freeway traffic. Journal de Physique I 2 (12), 2221-2229.

Newell, G. F., 1993. A simplified theory of kinematic waves in highway traffic, part i: general theory. Transportation Research Part B: Methodological 27 (4), 281-287.

Newell, G. F., 2002. A simplified car-following theory: a lower order model. Transportation Research Part B $36(3), 195-205$.

Rakha, H., Kamalanathsharma, R. K., 2011. Eco-driving at signalized intersections using v2i communication. In: Intelligent Transportation Systems (ITSC), 2011 14th International IEEE Conference on. IEEE, pp. $341-346$.

Richards, P. I., 1956. Shock Waves on the Highway . Operations Research 4 (1), 42-51.

Sanchez, M., Cano, J.-C., Kim, D., 2006. Predicting traffic lights to improve urban traffic fuel consumption. In: 2006 6th International Conference on ITS Telecommunications.

Schwarzkopf, A., Leipnik, R., 1977. Control of highway vehicles for minimum fuel consumption over varying terrain. Transportation Research 11 (4), 279-286. 
Shladover, S. E., Nowakowski, C., Cody, D., Bu, F., O'Connell, J., Spring, J., Dickey, S., Nelson, D., 2009. Effects of cooperative adaptive cruise control on traffic flow: Testing drivers' choices of following distances. Tech. Rep. UCB-ITS-PRR-2009-23, California PATH, Berkeley, CA.

Spiliopoulou, A. D., Papamichail, I., Papageorgiou, M., 2009. Toll plaza merging traffic control for throughput maximization. Journal of Transportation Engineering 136 (1), 67-76.

Stoer, J., Bulirsch, R., 2013. Introduction to numerical analysis. Vol. 12. Springer Science \& Business Media.

Tang, J., Song, Y., Miller, H. J., Zhou, X., 2015. Estimating the most likely space-time paths, dwell times and path uncertainties from vehicle trajectory data: A time geographic method. Transportation Research Part C: Emerging Technologies (in press).

URL http://www.sciencedirect.com/science/article/pii/S0968090X15003150

Tang, T., Shi, W., Shang, H., Wang, Y., 2014a. A new car-following model with consideration of inter-vehicle communication. Nonlinear Dynamics 76 (4), 2017-2023.

URL http://dx.doi.org/10.1007/s11071-014-1265-9

Tang, T.-Q., Shi, W.-F., Shang, H.-Y., Wang, Y.-P., 2014b. An extended car-following model with consideration of the reliability of inter-vehicle communication. Measurement 58, $286-293$.

URL http://www.sciencedirect.com/science/article/pii/S0263224114003856

Tielert, T., Killat, M., Hartenstein, H., Luz, R., Hausberger, S., Benz, T., 2010. The impact of traffic-lightto-vehicle communication on fuel consumption and emissions. In: Internet of Things (IOT), 2010. IEEE, pp. 1-8.

Tong, L., Zhou, X., Miller, H. J., 2015. Transportation network design for maximizing space-time accessibility. Transportation Research Part B: Methodological 81, 555-576.

Trayford, R., Doughty, B., van der Touw, J., 1984a. Fuel economy investigation of dynamic advisory speeds from an experiment in arterial traffic. Transportation Research Part A: General 18 (5), 415-419.

Trayford, R., Doughty, B., Wooldridge, M., 1984b. Fuel saving and other benefits of dynamic advisory speeds on a multi-lane arterial road. Transportation Research Part A: General 18 (5), 421-429.

Treiber, M., Kesting, A., Helbing, D., 2010. Three-phase traffic theory and two-phase models with a fundamental diagram in the light of empirical stylized facts. Transportation Research Part B: Methodological $44(8), 983-1000$.

Van Arem, B., van Driel, C., Visser, R., 2006. The impact of cooperative adaptive cruise control on traffic-flow characteristics. Intelligent Transportation Systems, IEEE Transactions on 7 (4), 429-436.

Von Stryk, O., Bulirsch, R., 1992. Direct and indirect methods for trajectory optimization. Annals of operations research $37(1), 357-373$.

Wang, M., Daamen, W., Hoogendoorn, S. P., van Arem, B., 2014a. Rolling horizon control framework for driver assistance systems. part i: Mathematical formulation and non-cooperative systems. Transportation Research Part C: Emerging Technologies 40, 271-289.

Wang, M., Daamen, W., Hoogendoorn, S. P., van Arem, B., 2014b. Rolling horizon control framework for driver assistance systems. part ii: Cooperative sensing and cooperative control. Transportation Research Part C: Emerging Technologies 40, 290-311.

Wu, G., Boriboonsomsin, K., Zhang, W.-B., Li, M., Barth, M., 2010. Energy and emission benefit comparison of stationary and in-vehicle advanced driving alert systems. Transportation Research Record: Journal of the Transportation Research Board (2189), 98-106. 
Wu, X., He, X., Yu, G., Harmandayan, A., Wang, Y., 2015. Energy-optimal speed control for electric vehicles on signalized arterials. Intelligent Transportation Systems, IEEE Transactions on PP (99), 1-11.

Yang, H., Jin, W.-L., 2014. A control theoretic formulation ofgreen driving strategies based on inter-vehicle communications. Transportation Research Part C: Emerging Technologies 41, 48-60.

Zohdy, I. H., Rakha, H. A., 2014. Intersection management via vehicle connectivity: The intersection cooperative adaptive cruise control system concept. Journal of Intelligent Transportation Systems (ahead-of-print), $1-16$. 